S. Ariki, A. Mathas and H. Rui

Nagoya Math. J.

Vol. 182 (2006), 47-134

\title{
CYCLOTOMIC NAZAROV-WENZL ALGEBRAS
}

\author{
SUSUMU ARIKI, ANDREW MATHAS AND HEBING RUI
}

On the occasion of Professor George Lusztig's $60^{\text {th }}$ birthday

\begin{abstract}
Nazarov [Naz96] introduced an infinite dimensional algebra, which he called the affine Wenzl algebra, in his study of the Brauer algebras. In this paper we study certain "cyclotomic quotients" of these algebras. We construct the irreducible representations of these algebras in the generic case and use this to show that these algebras are free of $\operatorname{rank} r^{n}(2 n-1)$ !! (when $\Omega$ is $\mathbf{u}$-admissible). We next show that these algebras are cellular and give a labelling for the simple modules of the cyclotomic Nazarov-Wenzl algebras over an arbitrary field. In particular, this gives a construction of all of the finite dimensional irreducible modules of the affine Wenzl algebra.
\end{abstract}

\section{$\S 1$. Introduction}

The Brauer algebras were introduced by Richard Brauer [Bra37] in his study of representations of the symplectic and orthogonal groups. In introducing these algebras Brauer was motivated by Schur's theory (see [Gre80]), which links the representation theory of the symmetric group $\mathfrak{S}_{n}$ and the general linear group GL( $V)$ via their commuting actions on "tensor space" $V^{\otimes n}$, where $\mathfrak{S}_{n}$ acts by place permutations. Analogously, the Brauer algebras are the centralizers of the image of a symplectic or orthogonal group in $\operatorname{End}\left(V^{\otimes n}\right)$, where $V$ is the defining representation of the group.

The Brauer algebras have now been studied by many authors and they have applications ranging from Lie theory, to combinatorics and knot the-

\footnotetext{
Received June 24, 2005.

2000 Mathematics Subject Classification: Primary 20C08; Secondary 16G99.

The first and second named authors enjoyed the hospitality of the Bernoulli Center at the EPFL Lausanne when they visited the program "Group representation theory" in June 2005. The paper was completed during their visit and the authors acknowledge partial support from the program. The second and third authors were supported, in part by Fellowships from the Japanese Society for the Promotion of Science. All three authors were supported by ARC grant "Quantized representation theory" during a visit to Sydney in January 2005. The third author was also supported by the Natural Science Foundation in China.
} 
ory; see, for example, [BW89], [Bro56], [DWH99], [Eny04], [FG95], [HR95], [HW89a], [HW89b], [Jon94], [LR97], [Mar96], [Naz96], [Ram95], [Rui05], [Ter01], [Wen88], [Wey97], [Xi00]. In this paper we are interested not so much in the Brauer algebra itself but in affine and cyclotomic analogues of it. Our starting point is a (special case of) Nazarov's [Naz96] affine Wenzl algebra $\mathscr{W}_{n}^{\text {aff }}(\Omega)$, an algebra which could legitimately be called the degenerate affine BMW algebra. Nazarov introduced the affine Wenzl algebra when studying the action of "Jucys-Murphy operators" on the irreducible representations of the Brauer algebras. Nazarov's idea was that the affine Wenzl algebra should play a similar role in the representation theory of the Brauer algebras to that played by the affine degenerate Hecke algebra of type A in the representation theory of the symmetric group.

Let $R$ be a commutative ring. The representation theory of the affine Wenzl algebras $\mathscr{W}_{n}^{\text {aff }}(\Omega)$, where $\Omega=\left\{\omega_{a} \in R \mid a \geq 0\right\}$, has not yet been studied. Motivated by the theory of the affine Hecke algebras and the cyclotomic Hecke algebras of type $G(r, 1, n)$ [Ari96], [DJM99], [Kle05] we introduce a "cyclotomic" quotient $\mathscr{W}_{r, n}(\mathbf{u})=\mathscr{W}_{n}^{\text {aff }}(\Omega) /\left\langle\prod_{i=1}^{r}\left(X_{1}-u_{i}\right)\right\rangle$ of $\mathscr{W}_{n}^{\text {aff }}(\Omega)$, which depends on an $r$-tuple of parameters $\mathbf{u}=\left(u_{1}, \ldots, u_{r}\right) \in R^{r}$. We call $\mathscr{W}_{r, n}(\mathbf{u})$ a cyclotomic Nazarov-Wenzl algebra. This paper develops the representation theory of the algebras $\mathscr{W}_{r, n}(\mathbf{u})$.

The first question that we are faced with is whether the cyclotomic Nazarov-Wenzl algebra $\mathscr{W}_{r, n}(\mathbf{u})$ is always free as an $R$-module. The Brauer algebra $\mathscr{B}_{n}$ is free of rank $(2 n-1) ! !=(2 n-1) \cdot(2 n-3) \cdots 3 \cdot 1$. We expect that the cyclotomic Nazarov-Wenzl algebra $\mathscr{W}_{r, n}(\mathbf{u})$ should be free of rank $r^{n}(2 n-1)$ !!. In Section 3, a detailed study of the representation theory of $\mathscr{W}_{r, 2}(\mathbf{u})$ shows that, in the semisimple case, $\mathscr{W}_{r, 2}(\mathbf{u})$ has rank $\left.r^{n}(2 n-1) ! !\right|_{n=2}$ if and only if $\Omega$ is $\mathbf{u}$-admissible (Definition 3.6). This constraint on $\Omega$ involves Schur's $q$-functions. Our first main result is the following.

TheOREM A. Let $R$ be a commutative ring in which 2 is invertible. Suppose that $\mathbf{u} \in R^{r}$ and that $\Omega$ is $\mathbf{u}$-admissible. Then the cyclotomic Nazarov-Wenzl algebra $\mathscr{W}_{r, n}(\mathbf{u})$ is free as an $R$-module of rank $r^{n}(2 n-1) ! !$.

The proof of this result occupies a large part of this paper. The idea behind the proof comes from [AK94]: for "generic" $R$ we explicitly construct a class of irreducible representations of $\mathscr{W}_{r, n}(\mathbf{u})$ and use them to show that the dimension of $\mathscr{W}_{r, n}(\mathbf{u}) / \operatorname{Rad} \mathscr{W}_{r, n}(\mathbf{u})$ is at least $r^{n}(2 n-1) ! !$. It is reasonably easy to produce a set of $r^{n}(2 n-1) !$ ! elements which span $\mathscr{W}_{r, n}(\mathbf{u})$, so 
this is enough to prove Theorem A. We construct these irreducible representations by giving "seminormal forms" for them (Theorem 4.13); that is, we give explicit matrix representations for the actions of the generators of $\mathscr{W}_{r, n}(\mathbf{u})$. The main difficulty in this argument is in showing that these matrices respect the relations of $\mathscr{W}_{r, n}(\mathbf{u})$, we do this using generating functions introduced by Nazarov [Naz96]. There is an additional subtlety in that we have to work over the real numbers in order to make a consistent choice of square roots in the representing matrices.

The next main result of the paper shows that $\mathscr{W}_{r, n}(\mathbf{u})$ is a cellular algebra in the sense of Graham and Lehrer [GL96]. This gives a lot of information about the representations of the cyclotomic Nazarov-Wenzl algebras. For example, cellularity implies that the decomposition matrix of $\mathscr{W}_{r, n}(\mathbf{u})$ is unitriangular.

Theorem B. Suppose that 2 is invertible in $R$ and that $\Omega$ is $\mathbf{u}$-admissible. Then the cyclotomic Nazarov-Wenzl algebra $\mathscr{W}_{r, n}(\mathbf{u})$ is a cellular algebra.

We prove Theorem B by constructing a cellular basis for $\mathscr{W}_{r, n}(\mathbf{u})$. We recall the definition of a cellular basis in Section 6 ; however, for the impatient experts we mention that the cell modules of $\mathscr{W}_{r, n}(\mathbf{u})$ are indexed by ordered pairs $(f, \lambda)$, where $0 \leq f \leq\left\lfloor\frac{n}{2}\right\rfloor$ and $\lambda$ is a multipartition of $n-2 f$, where $0 \leq f \leq\left\lfloor\frac{n}{2}\right\rfloor$, and the bases of the cell modules are indexed by certain ordered triples which are in bijection with the $n$-updown $\lambda$-tableaux.

Finally we consider the irreducible $\mathscr{W}_{r, n}(\mathbf{u})$-modules over a field $R$. The cell modules of $\mathscr{W}_{r, n}(\mathbf{u})$ have certain quotients $D^{(f, \lambda)}$, where $0 \leq f \leq\left\lfloor\frac{n}{2}\right\rfloor$ and $\lambda$ is a multipartition of $n-2 f$, which the theory of cellular algebras says are either zero or absolutely irreducible. Now, the cyclotomic NazarovWenzl algebra $\mathscr{W}_{r, n}(\mathbf{u})$ is filtered by two sided ideals with the degenerate Hecke algebras $\mathscr{H}_{r, n-2 f}(\mathbf{u})$ of type $G(r, 1, n-2 f)$ appearing as the successive quotients for $0 \leq f \leq\left\lfloor\frac{n}{2}\right\rfloor$. In Section 6 we show that the algebras $\mathscr{H}_{r, m}(\mathbf{u})$ are also cellular (in fact, this is the key to proving Theorem B); as a consequence, the irreducible $\mathscr{H}_{r, m}(\mathbf{u})$-modules are the non-zero modules $D^{\lambda}$, where $\lambda$ is a multipartition of $m$.

Using the results of the last paragraph we can construct all of the irreducible representations of the cyclotomic Nazarov-Wenzl algebras when $\Omega$ is admissible. This enables us to construct all finite dimensional representations of the affine Wenzl algebras over an algebraically closed field when 
$\Omega$ is admissible (Theorem 7.19). In the special case when $\omega_{0} \neq 0$ we also have the following classification of the irreducible $\mathscr{W}_{r, n}(\mathbf{u})$-modules.

Theorem C. Suppose that $R$ is a field in which 2 is invertible, that $\Omega$ is u-admissible and that $\omega_{0} \neq 0$. Then $\left\{D^{(f, \lambda)} \mid 0 \leq f \leq\left\lfloor\frac{n}{2}\right\rfloor, \lambda \vdash\right.$ $n-2 f$ and $\left.D^{\lambda} \neq 0\right\}$ is a complete set of pairwise non-isomorphic irreducible $\mathscr{W}_{r, n}(\mathbf{u})$-modules.

As an application of Theorem $\mathrm{C}$ we give necessary and sufficient conditions for $\mathscr{W}_{r, n}(\mathbf{u})$ to be quasi-hereditary when $R$ is a field and $\omega_{0} \neq 0$.

We note that Orellana and Ram [OR], building on [LR97], gave explicit formulae for the seminormal representations of the affine and cyclotomic BMW algebras [OR, Theorem 6.20]. As the (degenerate) affine Wenzl algebra is the degeneration of the affine BMW algebra, it is natural to expect that we should be able to derive the seminormal representations of $\mathscr{W}_{n}^{\text {aff }}(\Omega)$ from the results of $[\mathrm{OR}]$. Unfortunately, this is not possible because Orellana and Ram construct the seminormal representations only for a very restrictive class of cyclotomic BMW algebras corresponding to certain specializations of the parameters (see [OR, Theorem $6.17(\mathrm{c}-\mathrm{d})]$ ). These parameter choices are sufficient for the purposes of $[\mathrm{OR}]$, however, it is not clear that "enough" of these parameter choices are $\mathbf{u}$-admissible so we are unable to exploit [OR]. We also remark we had to work quite hard to ensure that we had made a consistent choice of square roots in our seminormal representations (cf. Assumption 4.12), and that it is not clear to us that [OR] have made a coherent choice of roots in their seminormal representations.

Another possible approach to the construction of the seminormal representations in this paper is via Jones' "basic construction" [Wen88]. Wenzl constructed the semisimple irreducible representations of the Brauer algebras this way. The final step of his argument used the non-degeneracy of the Markov trace to show that all partitions of $n$ are "permissible" (see [Wen88, Theorem 3.4]). We were unable to extend Wenzl's arguments to the cyclotomic Nazarov-Wenzl algebras because we were unable to prove that we have an analogous non-degenerate trace form. Note also that, a priori, it is not clear that $\mathscr{W}_{r, n-1}(\mathbf{u})$ is a subalgebra of $\mathscr{W}_{r, n}(\mathbf{u})$.

Finally, we remark that other variants of signed and cyclotomic Brauer algebras, G-Brauer algebras and cyclotomic BMW have been studied previously in the papers [CGW05], [GH], [HO01], [PK98], [PK02], [PS02], [RY04]. 


\section{Notational INDEX}

\begin{tabular}{|c|c|c|c|}
\hline$a_{\mathfrak{t}}(k)$ & eminormal matrix entry ........ 76 & $\mathscr{T}^{s t d}(\lambda)$ & Standard $\lambda$-tableaux .... \\
\hline$b_{\mathfrak{t}}(k)$ & eminormal matrix entry ...... 76 & $\mathscr{T}_{n}^{u d}(\lambda)$ & pdown $\lambda$-tableaux ..... \\
\hline$\xi_{n}(\omega)$ & r algebra $\ldots \ldots \ldots \ldots \ldots$ & $\mathbf{u}$ & ers for $\mathscr{W}_{r, n}(\mathbf{u}) \ldots$ \\
\hline $3(n)$ & diagrams .......55 & $\mathscr{W}_{r, n}(\mathbf{u})$ & Wenzl alg. . \\
\hline$B_{\gamma}$ & Element of $\mathscr{W}_{n}^{\text {aff }}(\lambda)$ or $\mathscr{W}_{r, n} \ldots \ldots 57$ & $\mathscr{W}_{r, n}^{f}$ & $=\mathscr{W}_{r, n} E^{f} \mathscr{W}_{r, n} \ldots \ldots$ \\
\hline$(k)$ & Jontent of $k$ in $\mathfrak{t} \ldots \ldots \ldots \ldots \ldots 73$ & & Two-sided ideal in $\mathscr{W}_{r, n}^{f}$ \\
\hline & ellular basis element of $\mathscr{W}_{r, n} \ldots .123$ & $\mathrm{ff}(\Omega)$ & Affine \\
\hline & $r, n \ldots \ldots \ldots 116$ & & $=\sum_{a}$ \\
\hline$E_{i}$ & $\ldots \ldots \ldots \ldots 51$ & $W_{k}(y)$ & $=\frac{1}{2}-y+\left(y-\frac{(-1)^{r}}{2}\right) \prod_{\alpha} \frac{y+c(\alpha)}{y-c(\alpha)}$. \\
\hline$E^{f}$ & $=E_{n-1} E_{n-3} \cdots E_{n-2 f+1} \cdots \cdots 113$ & $X_{j}$ & Generator of $\mathscr{W}_{n}^{\text {aff }}(\Omega) \ldots \ldots \ldots \ldots$ \\
\hline$\varepsilon_{f}$ & $\mathbb{W}_{1} \mathscr{W}_{r, n-2 f} \ldots \ldots \ldots 112$ & $X^{\alpha}$ & $X_{1}^{\alpha_{1}} \cdots X_{n}^{\alpha_{n}}$ \\
\hline & ntry ........75 & $Y_{j}$ & Gen \\
\hline $\mathscr{H}_{r, n} \mathbf{u}$ & & $\Delta(\lambda)$ & Sem \\
\hline$n_{\mathfrak{s t}}$ & ent of $\mathscr{H}_{r}$ & $\sigma_{f}$ & $f, n / \mathscr{W}_{r, n}^{f+1}$ \\
\hline$M_{\mathfrak{s t}}$ & .115 & & (2) \\
\hline$i$ & & & Cen \\
\hline $\mathfrak{u}(k)$ & & & \\
\hline & Generator of $\mathscr{H}_{r, n}(\mathbf{u}) \ldots \ldots$ & $\mathfrak{t} \ominus \mathfrak{u}$ & $\mathfrak{t} \backslash \mathfrak{u}$ or $\mathfrak{u} \backslash \mathfrak{t} \ldots \ldots \ldots \ldots \ldots$ \\
\hline
\end{tabular}

\section{§2. Affine and cyclotomic Nazarov-Wenzl algebras}

In [Naz96], Nazarov introduced an affine analogue of the Brauer algebra which he called the (degenerate) affine Wenzl algebra. The main objects of interest in this paper are certain "cyclotomic" quotients of Nazarov's algebra. In this section we define these algebras and prove some elementary results about them.

Fix a positive integer $n$ and a commutative ring $R$ with multiplicative identity $1_{R}$. Throughout this paper we will assume that 2 is invertible in $R$.

Definition 2.1. (Nazarov [Naz96, §4]) Fix $\Omega=\left\{\omega_{a} \mid a \geq 0\right\} \subseteq R$. The (degenerate) affine Wenzl algebra $\mathscr{W}_{n}^{\text {aff }}=\mathscr{W}_{n}^{\text {aff }}(\Omega)$ is the unital associative $R$-algebra with generators $\left\{S_{i}, E_{i}, X_{j} \mid 1 \leq i<n\right.$ and $\left.1 \leq j \leq n\right\}$ and relations

a) (Involutions) $S_{i}^{2}=1$, for $1 \leq i<n$.

b) (Affine braid relations)

(i) $S_{i} S_{j}=S_{j} S_{i}$ if $|i-j|>1$,

(ii) $S_{i} S_{i+1} S_{i}=S_{i+1} S_{i} S_{i+1}$, for $1 \leq i<n-1$,

(iii) $S_{i} X_{j}=X_{j} S_{i}$ if $j \neq i, i+1$.

c) (Idempotent relations) $E_{i}^{2}=\omega_{0} E_{i}$, for $1 \leq i<n$. 
d) (Commutation relations)

(i) $S_{i} E_{j}=E_{j} S_{i}$, if $|i-j|>1$,

(ii) $E_{i} E_{j}=E_{j} E_{i}$, if $|i-j|>1$,

(iii) $E_{i} X_{j}=X_{j} E_{i}$, if $j \neq i, i+1$,

(iv) $X_{i} X_{j}=X_{j} X_{i}$, for $1 \leq i, j \leq n$.

e) (Skein relations) $S_{i} X_{i}-X_{i+1} S_{i}=E_{i}-1$ and $X_{i} S_{i}-S_{i} X_{i+1}=E_{i}-1$, for $1 \leq i<n$.

f) (Unwrapping relations) $E_{1} X_{1}^{a} E_{1}=\omega_{a} E_{1}$, for $a>0$.

g) (Tangle relations)

(i) $E_{i} S_{i}=E_{i}=S_{i} E_{i}$, for $1 \leq i \leq n-1$,

(ii) $S_{i} E_{i+1} E_{i}=S_{i+1} E_{i}$, for $1 \leq i \leq n-2$,

(iii) $E_{i+1} E_{i} S_{i+1}=E_{i+1} S_{i}$, for $1 \leq i \leq n-2$.

h) (Untwisting relations) $E_{i+1} E_{i} E_{i+1}=E_{i+1}$ and $E_{i} E_{i+1} E_{i}=E_{i}$, for $1 \leq i \leq n-2$.

i) (Anti-symmetry relations) $E_{i}\left(X_{i}+X_{i+1}\right)=0$ and $\left(X_{i}+X_{i+1}\right) E_{i}=0$, for $1 \leq i<n$.

Our definition of $\mathscr{W}_{n}^{\text {aff }}$ differs from Nazarov's in two respects. First, Nazarov considers only the special case when $R=\mathbb{C}$; however, as we will indicate, most of the arguments that we need from [Naz96] go through without change when $R$ is an arbitrary ring. More significantly, Nazarov considers a more general algebra which is generated by the elements $\left\{S_{i}, E_{i}, X_{j}, \hat{\omega}_{a} \mid\right.$ $1 \leq i<n, 1 \leq j \leq n$ and $a \geq 0\}$ such that the $\hat{\omega}_{a}$ are central and the remaining generators satisfy the relations above. For our purposes it is more natural to define the elements $\omega_{a}$ to be elements of $R$ because without this assumption the cyclotomic quotients of $\mathscr{W}_{n}^{\text {aff }}$ would not be finite dimensional.

Note that $E_{i} E_{i+1} S_{i}=E_{i} E_{i+1} E_{i} S_{i+1}=E_{i} S_{i+1}$ and $S_{i+1} E_{i} E_{i+1}=$ $S_{i} E_{i+1} E_{i} E_{i+1}=S_{i} E_{i+1}$. Thus a quick inspection of the defining relations shows that $\mathscr{W}_{n}^{\text {aff }}$ has the following useful involution.

2.2. There is a unique $R$-linear anti-isomorphism $*: \mathscr{W}_{n}^{\text {aff }} \rightarrow \mathscr{W}_{n}^{\text {aff }}$ such that

$$
S_{i}^{*}=S_{i}, \quad E_{i}^{*}=E_{i} \quad \text { and } \quad X_{j}^{*}=X_{j}
$$

for all $1 \leq i<n$ and all $1 \leq j \leq n$. Moreover, $*$ is an involution. 
Using the defining relations it is not hard to see that $\mathscr{W}_{n}^{\text {aff }}$ is generated by the elements $S_{1}, \ldots, S_{n-1}, E_{1}, X_{1}$. There is no real advantage, however, to using this smaller set of generators as the corresponding relations are more complicated.

Lemma 2.3. (cf. [Naz96, (2.6)]) Suppose that $1 \leq i<n$ and that $a \geq 1$. Then

$$
S_{i} X_{i}^{a}=X_{i+1}^{a} S_{i}+\sum_{b=1}^{a} X_{i+1}^{b-1}\left(E_{i}-1\right) X_{i}^{a-b} .
$$

Proof. We argue by induction on $a$. When $a=1$ this is relation 2.1(e). If $a \geq 1$ then, by induction, we have

$$
\begin{aligned}
S_{i} X_{i}^{a+1} & =S_{i} X_{i}^{a} X_{i}=\left\{X_{i+1}^{a} S_{i}+\sum_{b=1}^{a} X_{i+1}^{b-1}\left(E_{i}-1\right) X_{i}^{a-b}\right\} X_{i} \\
& =X_{i+1}^{a} S_{i} X_{i}+\sum_{b=1}^{a} X_{i+1}^{b-1}\left(E_{i}-1\right) X_{i}^{a+1-b} .
\end{aligned}
$$

Now, by the skein relation $2.1(\mathrm{e}), S_{i} X_{i}=X_{i+1} S_{i}+E_{i}-1$, so

$$
\begin{aligned}
S_{i} X_{i}^{a+1} & =X_{i+1}^{a}\left(X_{i+1} S_{i}+E_{i}-1\right)+\sum_{b=1}^{a} X_{i+1}^{b-1}\left(E_{i}-1\right) X_{i}^{a+1-b} \\
& =X_{i+1}^{a+1} S_{i}+\sum_{b=1}^{a+1} X_{i+1}^{b-1}\left(E_{i}-1\right) X_{i}^{a+1-b}
\end{aligned}
$$

as required.

Corollary 2.4. Suppose that $a \geq 0$. Then

$$
\omega_{2 a+1} E_{1}=\frac{1}{2}\left\{-\omega_{2 a}+\sum_{b=1}^{2 a+1}(-1)^{b-1} \omega_{b-1} \omega_{2 a+1-b}\right\} E_{1} .
$$

Proof. Take $i=1$ and multiply the equation in Lemma 2.3 on the left and right by $E_{1}$. Since $S_{1} E_{1}=E_{1}=E_{1} S_{1}$, this gives

$$
E_{1} X_{1}^{a} E_{1}=E_{1} X_{2}^{a} E_{1}+\sum_{b=1}^{a} E_{1} X_{2}^{b-1}\left(E_{1}-1\right) X_{1}^{a-b} E_{1} .
$$


Since $E_{1} X_{1}^{c} E_{1}=\omega_{c} E_{1}, E_{1}\left(X_{1}+X_{2}\right)=0$ and $X_{1} X_{2}=X_{2} X_{1}$ we can rewrite this equation as

$$
\begin{aligned}
\omega_{a} E_{1} & =(-1)^{a} \omega_{a} E_{1}+\sum_{b=1}^{a}(-1)^{b-1} E_{1} X_{1}^{b-1}\left(E_{1}-1\right) X_{1}^{a-b} E_{1} \\
& =(-1)^{a} \omega_{a} E_{1}+\sum_{b=1}^{a}(-1)^{b-1}\left(E_{1} X_{1}^{b-1} E_{1} X_{1}^{a-b} E_{1}-E_{1} X_{1}^{a-1} E_{1}\right) \\
& =(-1)^{a} \omega_{a} E_{1}+\sum_{b=1}^{a}(-1)^{b-1}\left(\omega_{b-1} \omega_{a-b}-\omega_{a-1}\right) E_{1} \\
& =(-1)^{a} \omega_{a} E_{1}+\sum_{b=1}^{a}(-1)^{b-1} \omega_{b-1} \omega_{a-b} E_{1}+\sum_{b=1}^{a}(-1)^{b} \omega_{a-1} E_{1} .
\end{aligned}
$$

Setting $a=2 a^{\prime}+1$ proves the Corollary.

If we assume that $E_{1} \neq 0$ in $\mathscr{W}_{n}^{\text {aff }}$ and that $\mathscr{W}_{n}^{\text {aff }}$ is torsion free then this result says that the $\omega_{a}$, for $a$ odd, are determined by the $\omega_{b}$, for $b$ even.

Remark 2.5. If $a>0$ then the proof of the Corollary also gives the identity

$$
0=\left\{\sum_{b=1}^{2 a}(-1)^{b-1} \omega_{b-1} \omega_{2 a-b}\right\} E_{1} .
$$

However, this relation holds automatically because

$$
\begin{aligned}
\sum_{b=1}^{2 a}(-1)^{b-1} \omega_{b-1} \omega_{2 a-b} & =\sum_{b=1}^{a}(-1)^{b-1} \omega_{b-1} \omega_{2 a-b}+\sum_{b=a+1}^{2 a}(-1)^{b-1} \omega_{b-1} \omega_{2 a-b} \\
& =\sum_{b=1}^{a}(-1)^{b-1} \omega_{b-1} \omega_{2 a-b}+\sum_{b^{\prime}=1}^{a}(-1)^{2 a-b^{\prime}} \omega_{2 a-b^{\prime}} \omega_{b^{\prime}-1} \\
& =0 .
\end{aligned}
$$

Before we define the cyclotomic quotients of $\mathscr{W}_{n}^{\text {aff }}$, which are the main objects of study in this paper, we recall some standard definitions and notation from the theory of Brauer algebras and some of Nazarov's results.

A Brauer diagram on the $2 n$ vertices $\{1, \ldots, n, \overline{1}, \ldots, \bar{n}\}$ is a graph with $n$ edges such that each vertex lies on a (unique) edge. Equivalently, a Brauer diagram is a partitioning of $\{1, \ldots, n, \overline{1}, \ldots, \bar{n}\}$ into $n$ two element subsets. 
Let $\mathcal{B}(n)$ be the set of all Brauer diagrams on $\{1, \ldots, n, \overline{1}, \ldots, \bar{n}\}$. Then $\# \mathcal{B}(n)=(2 n-1) !$ !.

Let $\gamma \in \mathcal{B}(n)$ be a Brauer diagram. A vertical edge in $\gamma$ is any edge of the form $\{m, \bar{m}\}$, where $1 \leq m \leq n$. Horizontal edges are edges of the form $\{m, p\}$, or $\{\bar{m}, \bar{p}\}$, where $1 \leq m<p \leq n$.

For $i=1, \ldots, n-1$ let $\gamma(i, i+1)$ be the Brauer diagram with edges $\{i, \overline{i+1}\},\{i+1, \bar{i}\}$ and all other edges being vertical. Similarly, let $\gamma_{i}$ be the Brauer diagram with edges $\{i, i+1\},\{\bar{i}, \overline{i+1}\}$, and with all other edges being vertical. We set $s_{i}=b_{\gamma(i, i+1)}$ and $e_{i}=b_{\gamma_{i}}$. We also let $\gamma_{e}$ be the graph with edges $\{\{i, \bar{i}\} \mid 1 \leq i \leq n\}$.

Brauer diagrams can be represented diagrammatically as in the following examples. The vertices in the first rows are labelled from left to right as 1 to 4 , and the vertices in the second row are labelled $\overline{1}$ to $\overline{4}$.

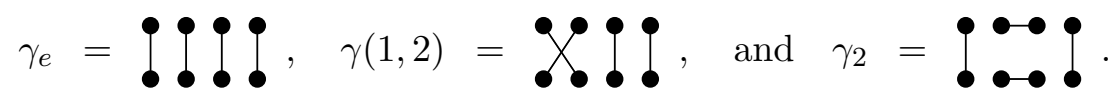

Given two Brauer diagrams $\gamma, \gamma^{\prime} \in \mathcal{B}(n)$ we define their product to be the diagram $\gamma \bullet \gamma^{\prime}$ which is obtained by identifying vertex $\bar{i}$ in $\gamma$ with vertex $i$ in $\gamma^{\prime}$, for $1 \leq i \leq n$. Let $\ell\left(\gamma, \gamma^{\prime}\right)$ be the number of loops in the graph $\gamma \bullet \gamma^{\prime}$ and let $\gamma \circ \gamma^{\prime}$ be the Brauer diagram obtained by deleting these loops. The following pictures give two examples of the multiplication $\gamma \circ \gamma^{\prime}$ of diagrams.

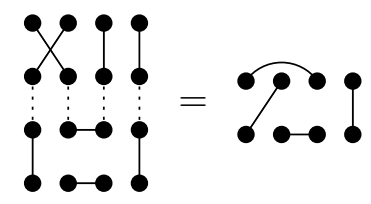

and

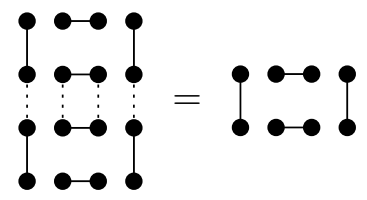

In the first example $\gamma=\gamma(1,2), \gamma^{\prime}=\gamma_{2}$ and $\ell\left(\gamma, \gamma^{\prime}\right)=0$. In the second example $\gamma=\gamma^{\prime}=\gamma_{2}$ and $\ell\left(\gamma, \gamma^{\prime}\right)=1$.

Recall that $R$ is a commutative ring.

Definition 2.6. (Brauer [Bra37]) Suppose that $\omega \in R$. The Brauer algebra $\mathscr{B}_{n}(\omega)$, with parameter $\omega$, is the $R$-algebra which is free as an $R$ module with basis $\left\{b_{\gamma} \mid \gamma \in \mathcal{B}(n)\right\}$ and with multiplication determined by

$$
b_{\gamma} b_{\gamma^{\prime}}=\omega^{\ell\left(\gamma, \gamma^{\prime}\right)} b_{\gamma \circ \gamma^{\prime}}
$$

for $\gamma, \gamma^{\prime} \in \mathcal{B}(n)$. 
It is easy to see that $\mathscr{B}_{n}(\omega)$ is an associative algebra with identity $b_{\gamma_{e}}$. We abuse notation and sometimes write $1=b_{\gamma_{e}}$.

The second example above indicates that $e_{i}^{2}=\omega e_{i}$, for $1 \leq i<n$. Similarly, $s_{i}^{2}=1$, for $1 \leq i<n$.

Let $\mathfrak{S}_{n}$ be the symmetric group on $n$ letters. To each permutation $w \in \mathfrak{S}_{n}$ we associate the Brauer diagram $\gamma(w)$ which has edges $\{\{i, \overline{w(i)}\} \mid$ for $1 \leq i \leq n\}$. Notice that if $w=(i, i+1)$ then this is consistent with the notation introduced above for the elements $s_{i}=b_{\gamma(i, i+1)} \in \mathscr{B}_{n}(\omega)$.

The diagrams $\left\{\gamma(w) \mid w \in \mathfrak{S}_{n}\right\}$ are precisely the Brauer diagrams which do not have any horizontal edges. It is easy to see that the map $w \mapsto b_{\gamma(w)}$ induces an algebra embedding of the group ring $R \mathfrak{S}_{n}$ of $\mathfrak{S}_{n}$ into $\mathscr{B}_{n}(\omega)$. In this way, $R \mathfrak{S}_{n}$ can be considered as a subalgebra of $\mathscr{B}_{n}(\omega)$.

There is a well-known presentation of $\mathscr{B}_{n}(\omega)$, which we now describe. See [MW00] for example.

Proposition 2.7. Suppose that $R$ is a commutative ring. The Brauer algebra $\mathscr{B}_{n}(\omega)$ is generated by the elements $s_{1}, \ldots, s_{n-1}, e_{1}, \ldots, e_{n-1}$ subject to the relations

$$
\begin{aligned}
& s_{i}^{2}=1, \\
& e_{i}^{2}=\omega e_{i}, \\
& s_{i} e_{i}=e_{i} s_{i}=e_{i}, \\
& s_{i} s_{j}=s_{j} s_{i}, \\
& s_{i} e_{j}=e_{j} s_{i}, \\
& e_{i} e_{j}=e_{j} e_{i}, \\
& s_{k} s_{k+1} s_{k}=s_{k+1} s_{k} s_{k+1} \text {, } \\
& e_{k} e_{k+1} e_{k}=e_{k}, \\
& e_{k+1} e_{k} e_{k+1}=e_{k+1} \text {, } \\
& s_{k} e_{k+1} e_{k}=s_{k+1} e_{k}, \\
& e_{k+1} e_{k} s_{k+1}=e_{k+1} s_{k} \text {, }
\end{aligned}
$$

where $1 \leq i, j<n$, with $|i-j|>1$, and $1 \leq k<n-1$.

Let $s_{i j}=b_{\gamma(i, j)}$, and let $e_{i j}=b_{\gamma_{i j}}$ where $\gamma_{i j}$ is the Brauer diagram with edges $\{i, j\},\{\bar{i}, \bar{j}\}$ and $\{k, \bar{k}\}$, for $k \neq i, j$.

Corollary 2.8. (Nazarov [Naz96, (2.2)]) Suppose that $\omega \in R$ and let $\Omega=\left\{\omega_{a} \mid a \geq 0\right\}$, where $\omega_{a}=\omega\left(\frac{\omega-1}{2}\right)^{a}$, for $a \geq 0$. Then there is a surjective algebra homomorphism $\pi: \mathscr{W}_{n}^{\text {aff }}(\Omega) \rightarrow \mathscr{B}_{n}(\omega)$ which is determined by

$$
\pi\left(S_{i}\right)=s_{i}, \quad \pi\left(E_{i}\right)=e_{i}, \quad \text { and } \quad \pi\left(X_{j}\right)=\frac{\omega-1}{2}+\sum_{k=1}^{j-1}\left(s_{k j}-e_{k j}\right),
$$

for $1 \leq i<n$ and $1 \leq j \leq n$. Moreover, $\operatorname{ker} \pi=\left\langle X_{1}-\left(\frac{\omega-1}{2}\right)\right\rangle$, so that

$$
\mathscr{W}_{n}^{\text {aff }}(\Omega) /\left\langle X_{1}-\left(\frac{\omega-1}{2}\right)\right\rangle \cong \mathscr{B}_{n}(\omega)
$$


Notice, in particular, that $\pi\left(X_{1}\right)=\frac{\omega-1}{2}$. To prove this result it is enough to show that the elements $\pi\left(X_{j}\right)$, for $1 \leq j \leq n$, satisfy the relations in $\mathscr{W}_{n}^{\text {aff }}(\Omega)$. For these calculations see [Naz96, Lemma 2.1 and Proposition 2.3].

Fix a Brauer diagram $\gamma \in \mathcal{B}(n)$. By Proposition 2.7 we can write $b_{\gamma}$ as a word in the generators $s_{1}, \ldots, s_{n-1}, e_{1}, \ldots, e_{n-1}$. Fix such a word for $b_{\gamma}$ and let $B_{\gamma} \in \mathscr{W}_{n}^{\text {aff }}(\Omega)$ be the corresponding word in the generators $S_{1}, \ldots, S_{n-1}, E_{1}, \ldots, E_{n-1}$. Then $\pi\left(B_{\gamma}\right)=b_{\gamma}$.

Given $\alpha, \beta \in \mathbb{N}_{0}^{n}$ and $\gamma \in \mathcal{B}(n)$ write

$$
X^{\alpha} B_{\gamma} X^{\beta}=X_{1}^{\alpha_{1}} \cdots X_{n}^{\alpha_{n}} B_{\gamma} X_{1}^{\beta_{1}} \cdots X_{n}^{\beta_{n}} .
$$

We want to use these monomials to give a basis of $\mathscr{W}_{n}^{\text {aff }}(\Omega)$. The antisymmetry relations $E_{i}\left(X_{i}+X_{i+1}\right)=0$, for $1 \leq i<n$, show that the set of all monomials is not linearly independent. In Theorem 2.12 below we will show that the following monomials are linearly independent.

Definition 2.9. Suppose that $\alpha, \beta \in \mathbb{N}_{0}^{n}$ and $\gamma \in \mathcal{B}(n)$. A monomial $X^{\alpha} B_{\gamma} X^{\beta}$ in $\mathscr{W}_{n}^{\text {aff }}(\Omega)$ is regular if

a) $\alpha_{r}=0$ whenever $r$ is the left endpoint of a horizontal edge in the top row of $\gamma$.

b) if $\beta_{l} \neq 0$ then $l$ is the left endpoint of a horizontal edge in the bottom row of $\gamma$.

We can view a regular monomial $X^{\alpha} B_{\gamma} X^{\beta}$ as a Brauer diagram if we colour the horizontal and vertical edges with the non-negative integers using $\alpha$ and $\beta$.

Following Corollary 2.4 we also make the following definition. (Recall that we are assuming that 2 is invertible in $R$.)

Definition 2.10. Let $\Omega=\left\{\omega_{a} \in R \mid a \geq 0\right\}$. Then $\Omega$ is admissible if

$$
\omega_{2 a+1}=\frac{1}{2}\left\{-\omega_{2 a}+\sum_{b=1}^{2 a+1}(-1)^{b-1} \omega_{b-1} \omega_{2 a+1-b}\right\},
$$

for all $a \geq 0$.

By Corollary $2.4 E_{1}$ is a torsion element if $\Omega$ is not admissible. 
Remark 2.11. Let $y$ be an indeterminate and consider the generating series $\widetilde{W}_{1}(y)=\sum_{a>0} \omega_{a} y^{-a}$. Then the condition for $\Omega$ to be admissible can be written as

$$
\left(\widetilde{W}_{1}(y)+y-\frac{1}{2}\right)\left(\widetilde{W}_{1}(-y)-y-\frac{1}{2}\right)=\left(\frac{1}{2}-y\right)\left(\frac{1}{2}+y\right) .
$$

Similar generating functions play an important role in Section 4 .

Theorem 2.12. (Nazarov [Naz96, Theorem 4.6]) Suppose $R$ is a commutative ring in which 2 is a unit and that $\Omega=\left\{\omega_{a} \in R \mid a \geq 0\right\}$ is admissible. Then $\mathscr{W}_{n}^{\text {aff }}(\Omega)$ is free as an $R$-module with basis $\left\{X^{\alpha} B_{\gamma} X^{\beta}\right.$ | $\alpha, \beta \in \mathbb{N}_{0}^{n}, \gamma \in \mathcal{B}(n)$, and $X^{\alpha} B_{\gamma} X^{\beta}$ is regular $\}$.

Sketch of proof. We have defined the elements of $\Omega$ to be scalars, but Nazarov [Naz96] works with a larger algebra $\widehat{\mathscr{W}_{n}^{\text {aff }}}(\widehat{\Omega})$ generated by elements $S_{i}, E_{i}, X_{j}$, for $1 \leq i<n$ and $1 \leq j \leq n$, and $\widehat{\Omega}=\left\{\widehat{\omega}_{a} \mid a \geq 0\right\}$ where these generators satisfy the same relations as the corresponding generators of $\mathscr{W}_{n}^{\text {aff }}(\Omega)$ except that the elements of $\Omega$ are central elements of $\widehat{\mathscr{W}_{n}^{\text {aff }}}(\widehat{\Omega})$, rather than scalars. Hence, $\mathscr{W}_{n}^{\text {aff }}(\Omega) \cong \widehat{\mathscr{W}}_{n}^{\text {aff }}(\widehat{\Omega}) / I$, where $I$ is the two sided ideal of $\widehat{\mathscr{W}_{n}^{\text {aff }}}(\widehat{\Omega})$ generated by the elements $\left\{\widehat{\omega}_{a}-\omega_{a} \mid a \geq 0\right\}$.

Nazarov puts a grading on $\widehat{\mathscr{W}_{n}^{\text {aff }}}(\widehat{\Omega})$ by setting $\operatorname{deg} S_{i}=\operatorname{deg} E_{i}=$ $\operatorname{deg} \widehat{\omega}_{a}=0$ and $\operatorname{deg} X_{i}=1$. To prove the result it is enough to work with the associated graded algebra $\operatorname{gr}\left(\widehat{\mathscr{W}_{n}^{\text {aff }}}(\widehat{\Omega})\right)$, where the grading is that induced by the degree function. The arguments of Lemma 4.4 and Lemma 4.5 from [Naz96] go through without change for an arbitrary ring, so $\widehat{\mathscr{W}_{n}^{\text {aff }}}(\widehat{\Omega})$ is spanned by

$$
\left\{\begin{array}{c|c}
X^{\alpha} B_{\gamma} X^{\beta} \widehat{\omega}_{2}^{h_{2}} \widehat{\omega}_{4}^{h_{4}} \ldots & \begin{array}{c}
\alpha, \beta \in \mathbb{N}_{0}^{n}, \gamma \in \mathcal{B}(n), h_{2 i} \geq 0, \text { for } i \geq 1, \\
\text { with only finitely many } h_{2 i} \neq 0
\end{array}
\end{array}\right\}
$$

where the monomials $X^{\alpha} B_{\gamma} X^{\beta}$ are all regular (see [Naz96, Theorem 4.6]). This implies that the regular monomials span $\mathscr{W}_{n}^{\text {aff }}(\Omega)$ for any ring $R$.

To complete the proof we first consider the case where the elements of $\Omega^{\prime}$ are indeterminates over $\mathbb{Z}$ and we consider the affine Wenzl algebras defined over the field $\mathbb{C}\left(\Omega^{\prime}\right)$ and over the ring $\mathbb{Z}\left[\Omega^{\prime}\right]$. We write $\mathscr{W}_{R, n}^{\text {aff }}\left(\Omega^{\prime}\right)=\mathscr{W}_{n}^{\text {aff }}\left(\Omega^{\prime}\right)$ to emphasize that $\mathscr{W}_{n}^{\text {aff }}\left(\Omega^{\prime}\right)$ is defined over the ring $R$.

Using Nazarov's algebra $\widehat{\mathscr{W}_{n}^{\text {aff }}}\left(\widehat{\Omega}^{\prime}\right)$ and arguing as above, it follows from [Naz96, Lemma 4.8] that the set of regular monomials are linearly independent when $R=\mathbb{C}\left(\Omega^{\prime}\right)$. By the last paragraph, the regular monomials span 
$\mathscr{W}_{\mathbb{Z}\left[\Omega^{\prime}\right], n}^{\text {aff }}\left(\Omega^{\prime}\right)$. Using the natural map $\mathscr{W}_{\mathbb{Z}\left[\Omega^{\prime}\right], n}^{\text {aff }}\left(\Omega^{\prime}\right) \rightarrow \mathscr{W}_{\mathbb{C}\left(\Omega^{\prime}\right), n}^{\text {aff }}\left(\Omega^{\prime}\right)$ it follows that $\mathscr{W}_{\mathbb{Z}\left[\Omega^{\prime}\right], n}^{\text {aff }}\left(\Omega^{\prime}\right)$ is free as a $\mathbb{Z}\left[\Omega^{\prime}\right]$-module and has basis the set of regular monomials. Hence, by a specialization argument, if $R$ is arbitrary $\operatorname{ring} R$ and $\Omega \subseteq R$ then

$$
\mathscr{W}_{R, q}^{\mathrm{aff}}(\Omega) \cong \mathscr{W}_{\mathbb{Z}\left[\Omega^{\prime}\right], n}^{\mathrm{aff}}\left(\Omega^{\prime}\right) \otimes_{\mathbb{Z}\left[\Omega^{\prime}\right]} R,
$$

where we consider $R$ as a $\mathbb{Z}\left[\Omega^{\prime}\right]$-module by letting $\omega_{a}^{\prime} \in \Omega^{\prime}$ act on $R$ as multiplication by $\omega_{a} \in \Omega$, for $a \geq 0$. Hence, $\mathscr{W}_{R, n}^{\text {aff }}(\Omega)$ is free as an $R$-module with basis the set of regular monomials as claimed.

We are now ready to define the cyclotomic Nazarov-Wenzl algebras. We assume henceforth that $\Omega$ is admissible.

Definition 2.13. Fix an integer $r \geq 1$ and $\mathbf{u}=\left(u_{1}, \ldots, u_{r}\right) \in R^{r}$. The cyclotomic Nazarov-Wenzl algebra $\mathscr{W}_{r, n}=\mathscr{W}_{r, n}(\mathbf{u})$ is the $R$-algebra $\mathscr{W}_{n}^{\mathrm{aff}}(\Omega) /\left\langle\left(X_{1}-u_{1}\right) \cdots\left(X_{1}-u_{r}\right)\right\rangle$.

We should write $\mathscr{W}_{r, n}(\mathbf{u}, \Omega)$, however, in Section 3 we will restrict to the case where $\Omega$ is $\mathbf{u}$-admissible (Definition 3.6), which implies that $\omega_{a}$ is determined by $\mathbf{u}$, for $a \geq 0$. For this reason we omit $\Omega$ from the notation for $\mathscr{W}_{r, n}(\mathbf{u})$.

By Corollary 2.8 the Brauer algebras $\mathscr{B}_{n}(\omega)$ are a special case of the cyclotomic Nazarov-Wenzl algebras corresponding to $r=1$ and $\Omega=$ $\left\{\omega\left(\frac{\omega-1}{2}\right)^{a} \mid a \geq 0\right\}$.

By definition there is a surjection $\pi_{r, n}: \mathscr{W}_{n}^{\text {aff }}(\Omega) \rightarrow \mathscr{W}_{r, n}(\mathbf{u})$. Abusing notation, we write $S_{i}=\pi_{r, n}\left(S_{i}\right), E_{i}=\pi_{r, n}\left(E_{i}\right), X_{j}=\pi_{r, n}\left(X_{j}\right)$, and $B_{\gamma}=$ $\pi_{r, n}\left(B_{\gamma}\right)$ for $1 \leq i<n, 1 \leq j \leq n$ and $\gamma \in \mathcal{B}(n)$.

Notice that because $\left(X_{1}-u_{1}\right) \cdots\left(X_{1}-u_{r}\right)=0$ in $\mathscr{W}_{r, n}(\mathbf{u})$ the cyclotomic Nazarov-Wenzl algebras have only $r$ unwrapping relations; that is, we only need to impose the relations $E_{1} X_{1}^{a} E_{1}=\omega_{a} E_{1}$, for $0 \leq a \leq r-1$.

Every $\mathscr{W}_{r, n}(\mathbf{u})$-module can be considered as a $\mathscr{W}_{n}^{\text {aff }}(\bar{\Omega})$-module by inflation along the surjection $\pi_{r, n}: \mathscr{W}_{n}^{\text {aff }}(\Omega) \rightarrow \mathscr{W}_{r, n}(\mathbf{u})$. In particular, every irreducible $\mathscr{W}_{r, n}(\mathbf{u})$-module is also an irreducible $\mathscr{W}_{n}^{\text {aff }}(\Omega)$-module. Conversely, it is not hard to see that every irreducible $\mathscr{W}_{n}^{\text {aff }}(\Omega)$-module $M$ over an algebraically closed field can be considered as an irreducible module for some cyclotomic Nazarov-Wenzl algebra $\mathscr{W}_{r, n}(\mathbf{u})$, where $\mathbf{u}$ depends on $\Omega$ and $M$. At first sight this is not very useful because almost all of the results in this paper require that $\Omega$ be $\mathbf{u}$-admissible (Definition 3.6) and, in general, it seems unlikely that $\Omega$ will be $\mathbf{u}$-admissible for all of the parameters $\mathbf{u}$ that 
arise in this way. Nevertheless, this observation and the theory of cellular algebras allows us to construct all of the finite dimensional $\mathscr{W}_{n}^{\text {aff }}(\Omega)$ modules over an algebraically closed field when $\Omega$ is admissible; see Theorem 7.19.

For our first result about the cyclotomic Nazarov-Wenzl algebras we prove the easy half of Theorem A. That is, we show that $\mathscr{W}_{r, n}(\mathbf{u})$ is spanned by $r^{n}(2 n-1) !$ ! elements.

Definition 2.14. Suppose that $\alpha, \beta \in \mathbb{N}_{0}^{n}$ and $\gamma \in \mathcal{B}(n)$.

a) The monomial $X^{\alpha} B_{\gamma} X^{\beta}$ in $\mathscr{W}_{r, n}(\mathbf{u})$ is regular if $X^{\alpha} B_{\gamma} X^{\beta}$ is a regular monomial in $\mathscr{W}_{n}^{\text {aff }}(\Omega)$.

b) The monomial $X^{\alpha} B_{\gamma} X^{\beta}$ in $\mathscr{W}_{r, n}(\mathbf{u})$ is $r$-regular if it is regular and $0 \leq \alpha_{i}, \beta_{i}<r$, for all $1 \leq i \leq n$.

Proposition 2.15. The cyclotomic Nazarov-Wenzl algebra $\mathscr{W}_{r, n}(\mathbf{u})$ is spanned by the set of r-regular monomials $\left\{X^{\alpha} B_{\gamma} X^{\beta}\right\}$. In particular, if $R$ is a field then

$$
\operatorname{dim}_{R} \mathscr{W}_{r, n}(\mathbf{u}) \leq r^{n}(2 n-1) ! !
$$

Proof. By Theorem 2.12, and the definitions, $\mathscr{W}_{r, n}(\mathbf{u})$ is spanned by the regular monomials in $\mathscr{W}_{r, n}(\mathbf{u})$. As in the proof of Theorem 2.12, we put a grading on $\mathscr{W}_{r, n}(\mathbf{u})$. Then in the associated graded algebra, gr $\mathscr{W}_{r, n}(\mathbf{u})$, we have the relation $\left(X_{i}-u_{1}\right) \cdots\left(X_{i}-u_{r}\right)=0$. We claim that the regular monomial $X^{\alpha} B_{\gamma} X^{\beta}$ can be written as a linear combination of $r$-regular monomials. If $X^{\alpha} B_{\gamma} X^{\beta}$ is an $r$-regular monomial then there is nothing to prove so we may assume that $X^{\alpha} B_{\gamma} X^{\beta}$ is not $r$-regular and, in particular, that $|\alpha|+|\beta|>0$. Then, using the relation $\left(X_{i}-u_{1}\right) \cdots\left(X_{i}-u_{r}\right)=0$ we can subtract a linear combination of $r$-regular monomials from $X^{\alpha} B_{\gamma} X^{\beta}$ to obtain a linear combination of regular elements of smaller degree. The claim now follows by induction.

Finally, a counting argument shows that the number of $r$-regular monomials is equal to $r^{n}(2 n-1)$ !!. Therefore, if $R$ is a field then $\operatorname{dim}_{R} \mathscr{W}_{r, n}(\mathbf{u}) \leq$ $r^{n}(2 n-1) ! !$.

The degenerate (cyclotomic) Hecke algebra $\mathscr{H}_{r, n}(\mathbf{u})$ of type $G(r, 1, n)$ is the unital associative $R$-algebra with generators $T_{1}, \ldots, T_{n-1}, Y_{1}, \ldots, Y_{n}$ 
and relations

$$
\begin{aligned}
\left(Y_{1}-u_{1}\right) \cdots\left(Y_{1}-u_{r}\right) & =0, & T_{i}^{2} & =1, \\
T_{i} T_{j} & =T_{j} T_{i}, & Y_{i} Y_{k} & =Y_{k} Y_{i}, \\
T_{i} Y_{i}-Y_{i+1} T_{i} & =-1, & Y_{i} T_{i}-T_{i} Y_{i+1} & =-1, \\
T_{j} T_{j+1} T_{j} & =T_{j+1} T_{j} T_{j+1}, & T_{i} Y_{\ell} & =Y_{\ell} T_{i}
\end{aligned}
$$

for $1 \leq i<n, 1 \leq j<n-1$ with $|i-j|>1$, and $1 \leq k \leq n, \ell \neq i, i+1$. Therefore there is a surjective algebra homomorphism $\mathscr{W}_{r, n}(\mathbf{u}) \rightarrow \mathscr{H}_{r, n}(\mathbf{u})$ determined by

$$
S_{i} \longmapsto T_{i}, \quad E_{i} \longmapsto 0, \quad \text { and } \quad X_{j} \longmapsto Y_{j}
$$

for $1 \leq i<n$ and $1 \leq j \leq n$. (In fact, a special case of Proposition 7.2 below shows that $\mathscr{H}_{r, n}(\mathbf{u}) \cong \mathscr{W}_{r, n}(\mathbf{u}) /\left\langle E_{1}\right\rangle$.) Consequently, every irreducible $\mathscr{H}_{r, n}(\mathbf{u})$-module can be considered as an irreducible $\mathscr{W}_{r, n}(\mathbf{u})$-module via inflation. These irreducible modules are precisely the irreducibles upon which $E_{i}$ acts as zero. We record this fact for future use.

Corollary 2.16. Suppose that $R$ is a field and that $M$ is an irreducible $\mathscr{W}_{r, n}(\mathbf{u})$-module which is annihilated by some $E_{i}$. Then $M$ is an irreducible $\mathscr{H}_{r, n}(\mathbf{u})$-module.

Proof. As $E_{i+1}=S_{i} S_{i+1} E_{i} S_{i+1} S_{i}$ and $S_{j}$ is invertible for all $j$, the two-sided ideal of $\mathscr{W}_{r, n}(\mathbf{u})$ generated by $E_{1}$ is the same as the two-sided ideal generated by $E_{i}$, for $1 \leq i<n$. The result now follows from the remarks above.

Recall that the degenerate affine Hecke algebra is a finitely generated module over its center (see, for example, [Kle05]), which is the ring of the symmetric polynomials in $Y_{1}, \ldots, Y_{n}$. This fact, together with Dixmier's version of Schur's lemma, implies that all of the irreducible modules of the degenerate affine Hecke algebra are finite dimensional.

By the last paragraph the power sum symmetric functions are central elements of the degenerate affine Hecke algebra. In contrast, only the power sums of odd degree are central in $\mathscr{W}_{n}^{\text {aff }}(\Omega)$. Another difference is that the affine Wenzl algebra is not finitely generated over its center. To see this, we give an example of an infinite dimensional irreducible $\mathscr{W}_{2}^{\text {aff }}(\Omega)$-module. 
ExAMPLE 2.17. Suppose that $\Omega$ is admissible and that $\widetilde{W}_{1}(y)=$ $\sum_{a \geq 0} \omega_{a} y^{-a}$ is not a rational function in $y$. Consider $V=\bigoplus_{n \geq 0} R v_{n}$. Define an action of $\mathscr{W}_{2}^{\text {aff }}(\Omega)$ on $V$ by $E v_{n}=\omega_{n} v_{0}, X_{1} v_{n}=v_{n+1}, X_{2} v_{n}=-v_{n+1}$ and

$$
S v_{n}=(-1)^{n} v_{n}-\varepsilon v_{n-1}+\sum_{k=0}^{n-1}(-1)^{k} \omega_{n-k-1} v_{k},
$$

where $\varepsilon=1$, if $n \equiv 1(\bmod 2)$, and $\varepsilon=0$, otherwise. All of the defining relations except for the relation $S^{2}=1$ are easy to check. As $S^{2}$ commutes with $X_{1}, S^{2} v_{0}=v_{0}$ and $X_{1} v_{n}=v_{n+1}$, we have that $S^{2}$ acts as the identity on $V$.

Now we show that $V$ is irreducible. Let $W$ be a $\mathscr{W}_{2}^{\text {aff }}(\Omega)$-submodule of $V$. Suppose that $E W=0$. If $\sum c_{n} v_{n} \in W$ then $\sum c_{n} \omega_{n+k}=0$, for all $k \geq 0$. As the vectors $\left\{\left(\omega_{k}, \omega_{k+1}, \ldots\right) \in R^{\infty} \mid k \geq 0\right\}$ span an infinite dimensional subspace of $R^{\infty}$, we have $c_{n}=0$, for all $n \geq 0$. Hence $W=0$. Thus, $W \neq 0$ implies $E W \neq 0$. Then $v_{0} \in W$ and $W=V$. Therefore, $V$ is an irreducible $\mathscr{W}_{2}^{\text {aff }}(\Omega)$-module as claimed.

In light of this example, we restrict our attention to finite dimensional $\mathscr{W}_{n}^{\text {aff }}(\Omega)$-modules in what follows.

\section{§3. Restrictions on $\Omega$ and the irreducible representations of $\mathscr{W}_{r, 2}$}

In this section we explicitly compute the (possible) irreducible representations of the cyclotomic Nazarov-Wenzl algebras $\mathscr{W}_{r, 2}(\mathbf{u})$. As a consequence we find a set of conditions on the parameter set $\Omega$ which ensure that $\mathscr{W}_{r, 2}(\mathbf{u})$ has dimension $3 r^{2}=\left.r^{n}(2 n-1) ! !\right|_{n=2}$ when $R$ is a field. In the next section we will see that these conditions on $\Omega$ are exactly what we need for general $n$.

The cyclotomic Nazarov-Wenzl algebra $\mathscr{W}_{r, 2}(\mathbf{u})$ is generated by $S_{1}, E_{1}$, $X_{1}$ and $X_{2}$. Throughout this section we suppose that $R$ is an algebraically closed field and, for convenience, we set $S=S_{1}$ and $E=E_{1}$.

Proposition 3.1. Suppose that $M$ is an irreducible $\mathscr{W}_{r, 2}(\mathbf{u})$-module such that $E M=0$. Then either:

a) $M=R m$ is one dimensional and the action of $\mathscr{W}_{r, 2}(\mathbf{u})$ is determined by

$$
S m=\varepsilon m, \quad E m=0, \quad X_{1} m=u_{i} m, \quad \text { and } \quad X_{2} m=\left(u_{i}+\varepsilon\right) m,
$$


where $\varepsilon= \pm 1$ and $1 \leq i \leq r$. In particular, up to isomorphism, there are at most $2 r$ such representations.

b) $M$ is two dimensional and the action of $\mathscr{W}_{r, 2}(\mathbf{u})$ is given by

$$
S \mapsto \frac{1}{u_{i}-u_{j}}\left(\begin{array}{cc}
-1 & b \\
c & 1
\end{array}\right), \quad E \mapsto\left(\begin{array}{cc}
0 & 0 \\
0 & 0
\end{array}\right), X_{1} \mapsto\left(\begin{array}{cc}
u_{i} & 0 \\
0 & u_{j}
\end{array}\right), \text { and } X_{2} \mapsto\left(\begin{array}{cc}
u_{j} & 0 \\
0 & u_{i}
\end{array}\right) \text {, }
$$

for some non-zero $b, c \in R$ such that $b c=\left(u_{i}-u_{j}\right)^{2}-1$, where $u_{i} \neq u_{j}$. Up to isomorphism there are at most $\left(\begin{array}{l}r \\ 2\end{array}\right)$ such representations.

c) $M$ is two dimensional and the action of $\mathscr{W}_{r, 2}(\mathbf{u})$ is given by

$$
S \mapsto\left(\begin{array}{ll}
0 & 1 \\
1 & 0
\end{array}\right), \quad E \mapsto\left(\begin{array}{ll}
0 & 0 \\
0 & 0
\end{array}\right), \quad X_{1} \mapsto\left(\begin{array}{cc}
u_{i} & -1 \\
0 & u_{i}
\end{array}\right), \quad \text { and } \quad X_{2} \mapsto\left(\begin{array}{cc}
u_{i} & 1 \\
0 & u_{i}
\end{array}\right) \text {. }
$$

Up to isomorphism there are at most $r$ such representations.

Proof. As noted in Corollary 2.16 $M$ is an irreducible $\mathscr{H}_{r, 2}(\mathbf{u})$-module. The result now follows from the representation theory of $\mathscr{H}_{r, 2}(\mathbf{u})$ : choose a simultaneous eigenvector $m$ of $R\left[Y_{1}, Y_{2}\right]$. Then, because $\mathscr{H}_{r, 2}(\mathbf{u})=$ $R\left[Y_{1}, Y_{2}\right]+T_{1} R\left[Y_{1}, Y_{2}\right]$, if $M$ is not one dimensional then it must be two dimensional. If this is the case, $\{m, S m\}$ is a basis of $M$. Further, if the eigenvalues for the action $Y_{1}$ on $M$ are distinct, then we can simultaneously diagonalize $Y_{1}$ and $Y_{2}$. All of our claims now follow.

Note that since $\prod_{i=1}^{r}\left(X_{1}-u_{i}\right)$ acts as zero on $M$, case (c) can only arise if the $u_{i}$ are not pairwise distinct. The irreducible representations of $\mathscr{W}_{r, 2}(\mathbf{u})$ upon which $E$ acts non-trivially take more effort to understand.

Proposition 3.2. Let $F$ be a field in which 2 is invertible and that $u_{1}, \ldots, u_{r}$ are algebraically independent over $F$. Let $R=F\left(u_{1}, \ldots, u_{r}\right)$ and let $\mathscr{W}_{r, 2}(\mathbf{u})$ be the cyclotomic Nazarov-Wenzl algebra defined over $R$, where $\omega_{0} \neq 0$. Then $\mathscr{W}_{r, 2}(\mathbf{u})$ has a unique irreducible module $M$ such that $E M \neq 0$. Moreover, if $d=\operatorname{dim}_{R} M$ then $d \leq r$ and there exists a basis $\left\{m_{1}, \ldots, m_{d}\right\}$ of $M$ and scalars $\left\{v_{1}, \ldots, v_{d}\right\} \subseteq\left\{u_{1}, \ldots, u_{r}\right\}$, with $v_{i} \neq v_{j}$ when $i \neq j$, such that for $1 \leq i \leq d$ the following hold:

a) $X_{1} m_{i}=v_{i} m_{i}$ and $X_{2} m_{i}=-v_{i} m_{i}$,

b) $E m_{i}=\gamma_{i}\left(m_{1}+\cdots+m_{d}\right)$ and

c) $S m_{i}=\frac{\gamma_{i}-1}{2 v_{i}} m_{i}+\sum_{j \neq i} \frac{\gamma_{i}}{v_{i}+v_{j}} m_{j}$ 
where $\gamma_{i}=\left(2 v_{i}-(-1)^{d}\right) \prod_{\substack{1 \leq j \leq d \\ j \neq i}} \frac{v_{i}+v_{j}}{v_{i}-v_{j}}$. Moreover, $\omega_{a}=\sum_{j=1}^{d} v_{j}^{a} \gamma_{j}$, for all $a \geq 0 ;$ and, in particular,

$$
\omega_{0}= \begin{cases}2\left(v_{1}+\cdots+v_{d}\right), & \text { if } d \text { is even } \\ 2\left(v_{1}+\cdots+v_{d}\right)+1, & \text { if } d \text { is odd }\end{cases}
$$

Conversely, if $\omega_{a}=\sum_{j=1}^{d} v_{j}^{a} \gamma_{j}$, for all $a \geq 0$, then $(a)-(c)$ define a $\mathscr{W}_{r, 2}(\mathbf{u})$ module $M$ with $E M \neq 0$.

Proof. Suppose that $M$ is an irreducible $\mathscr{W}_{r, 2}$-module such that $E M \neq$ 0 . Note that $M$ is finite dimensional. Let $d=\operatorname{dim}_{R} M$. We first show that (a)-(c) hold. Since $u_{1}, \ldots, u_{r}$ are pairwise distinct, we can fix a basis $\left\{m_{1}, \ldots, m_{d}\right\}$ of $M$ consisting of eigenvectors for $X_{1}$. Write $X_{1} m_{i}=v_{i} m_{i}$, for some $v_{i} \in\left\{u_{1}, \ldots, u_{r}\right\}$.

Set $f:=\frac{1}{\omega_{0}} E$. This is a non-zero idempotent and $f M \neq 0$ since $E M \neq 0$.

Fix an element $0 \neq m \in f M$. Then $E m=\omega_{0} m$ and $S m=m$ (since $S E=E)$. As $0=\left(X_{1}+X_{2}\right) E m=\left(X_{1}+X_{2}\right) \omega_{0} m$, we have $\left(X_{1}+X_{2}\right) m=0$. However, $X_{1}+X_{2}$ is central in $\mathscr{W}_{r, 2}$, so $X_{1}+X_{2}$ acts as a scalar on $M$ by Schur's lemma. Hence, $X_{2} m_{i}=-X_{1} m_{i}=-v_{i} m_{i}$, for $i=1, \ldots, d$, proving (a).

We claim that $\left\{m, X_{1} m, \ldots, X_{1}^{d-1} m\right\}$ is a basis of $M$. To see this, for any $a \geq 0$ let $M_{a}$ be the $R$-submodule of $M$ spanned by $\left\{m, X_{1} m, \ldots, X_{1}^{a} m\right\}$. Notice that $M_{a}$ is closed under left multiplication by $E$ since if $k \geq 0$ then

$$
E X_{1}^{k} m=E X_{1}^{k} f m=\frac{1}{\omega_{0}} E X_{1}^{k} E m=\frac{\omega_{k}}{\omega_{0}} E m=\omega_{k} m
$$

Also, by Lemma 2.3,

$$
\begin{aligned}
S X_{1}^{a} m & =\left(X_{2}^{a} S+\sum_{b=1}^{a} X_{2}^{b-1}(E-1) X_{1}^{a-b}\right) m \\
& =X_{2}^{a} m+\sum_{b=1}^{a}\left(X_{2}^{b-1} E X_{1}^{a-b} E \frac{1}{\omega_{0}} m-X_{1}^{a-b} X_{2}^{b-1} m\right) \\
& =X_{2}^{a} m+\sum_{b=1}^{a}\left(\frac{\omega_{a-b}}{\omega_{0}} X_{2}^{b-1} E m-X_{1}^{a-b} X_{2}^{b-1} m\right) \\
& =(-1)^{a} X_{1}^{a} m+\sum_{b=1}^{a}\left((-1)^{b-1} \omega_{a-b} X_{1}^{b-1} m-(-1)^{b-1} X_{1}^{a-1} m\right) .
\end{aligned}
$$


So, $M_{a}$ is closed under multiplication by $S$. Choose $a \geq 0$ to be minimal such that $\left\{m, X_{1} m, \ldots, X_{1}^{a+1} m\right\}$ is not linearly independent. Since $X_{1}^{a+1} m \in M_{a}, M_{a}$ is closed under multiplication by $X_{1}$. Hence, $M_{a}=M$ since $M$ is irreducible. By counting dimensions, $M=M_{d-1}$, proving the claim.

Next we show that $E M=R m$. Suppose that $m^{\prime}=\sum_{i=0}^{d-1} c_{i} X_{1}^{i} m \in$ EM. Then

$$
m^{\prime}=\frac{1}{\omega_{0}} E m^{\prime}=\frac{1}{\omega_{0}} \sum_{i=0}^{d-1} c_{i} E X_{1}^{i} m=\frac{1}{\omega_{0}^{2}} \sum_{i=0}^{d-1} c_{i} E X_{1}^{i} E m=\frac{1}{\omega_{0}^{2}}\left(\sum_{i=0}^{d-1} c_{i} \omega_{i}\right) m,
$$

since $E a=\omega_{0} a$ whenever $a \in E M$. Hence, $E M=R m$, as claimed.

Recall that we have fixed a basis $\left\{m_{1}, \ldots, m_{d}\right\}$ of $M$. Write $m=$ $\sum_{i=1}^{d} r_{i} m_{i}$, for some $r_{i} \in R$. Suppose that $r_{i}=0$ for some $i$. Then

$$
\prod_{\substack{1 \leq j \leq d \\ j \neq i}}\left(X_{1}-v_{j}\right) \cdot m=0
$$

This contradicts the linear independence of $\left\{m, X_{1} m, \ldots, X_{1}^{d-1} m\right\}$; hence, $r_{i} \neq 0$ for $i=1, \ldots, d$. By rescaling the $m_{i}$, if necessary, we can and do assume that $m=m_{1}+\cdots+m_{d}$ in the following.

By the argument of the last paragraph, all of the eigenvalues $\left\{v_{1}, \ldots, v_{d}\right\}$ of $m$ must be distinct. This also shows that $d=\operatorname{dim} M \leq r$ and that $\left\{v_{1}, \ldots, v_{d}\right\}$ are algebraically independent (since we are assuming that $u_{1}, \ldots, u_{r}$ are algebraically independent). In particular, $v_{i}$ and $v_{i}+v_{j}$, for $i \neq j$, are invertible. So the formula in part (c) makes sense.

As $E M=R m$, we can define elements $\gamma_{i} \in R$ by

$$
E m_{i}=\gamma_{i} m=\gamma_{i}\left(m_{1}+\cdots+m_{d}\right), \quad \text { for } i=1, \ldots, d .
$$

Write $S m_{i}=\sum_{j=1}^{d} c_{j}^{(i)} m_{j}$. Then $X_{1} S m_{i}-S X_{2} m_{i}=(E-1) m_{i}$ reads

$$
\sum_{j=1}^{d} c_{j}^{(i)} v_{j} m_{j}+v_{i}\left(\sum_{j=1}^{d} c_{j}^{(i)} m_{j}\right)=\gamma_{i}\left(m_{1}+\cdots+m_{d}\right)-m_{i}
$$

Thus, $\left(v_{i}+v_{j}\right) c_{j}^{(i)}=\gamma_{i}-\delta_{i j}$ and we have

$$
S m_{i}=\frac{\gamma_{i}-1}{2 v_{i}} m_{i}+\sum_{j \neq i} \frac{\gamma_{i}}{v_{i}+v_{j}} m_{j}
$$


This proves (c).

Next we prove the formula for $\gamma_{i}$ given in (b). Since $E=S E$ we find that

$$
\gamma_{i} \sum_{j=1}^{d} m_{j}=E m_{i}=S E m_{i}=\gamma_{i} \sum_{j=1}^{d}\left\{\frac{\gamma_{j}-1}{2 v_{j}}+\sum_{k \neq j} \frac{\gamma_{k}}{v_{j}+v_{k}}\right\} m_{j},
$$

for $i=1, \ldots, r$. Note that some $\gamma_{i}$ is non-zero, since $E M \neq 0$. Thus, comparing the coefficient of $m_{j}$ on both sides shows that

$$
\sum_{k=1}^{d} \frac{\gamma_{k}}{v_{j}+v_{k}}=1+\frac{1}{2 v_{j}}
$$

for $j=1, \ldots, d$.

We claim that

$$
\operatorname{det}\left(\frac{1}{v_{i}+v_{j}}\right)_{1 \leq i, j \leq d}=\left(\prod_{i=1}^{d} 2 v_{i}\right)^{-1} \prod_{i>j}\left(\frac{v_{i}-v_{j}}{v_{i}+v_{j}}\right)^{2}
$$

To see this, observe that

$$
\left(\prod_{i=1}^{d} 2 v_{i}\right) \prod_{i>j}\left(v_{i}+v_{j}\right)^{2} \operatorname{det}\left(\frac{1}{v_{i}+v_{j}}\right)_{1 \leq i, j \leq d}
$$

is a symmetric polynomial in $v_{1}, \ldots, v_{d}$ which is divisible by $v_{i}-v_{j}$ for $i \neq j$. This shows that this determinant is a constant multiple of $\prod_{i>j}\left(v_{i}-v_{j}\right)^{2}$. To determine the constant, we multiply $\operatorname{det}\left(\frac{1}{v_{i}+v_{j}}\right)_{1 \leq i, j \leq d}$ by $v_{n}$, set $v_{n}=\infty$ and use induction.

By the last paragraph, the matrix $\left(\frac{1}{v_{i}+v_{j}}\right)_{1 \leq i, j \leq d}$ is invertible, so $\gamma_{1}, \ldots, \gamma_{d}$ are uniquely determined. Hence, to prove the formula for $\gamma_{i}$ it suffices to show that

$$
\sum_{k=1}^{d} \frac{2 v_{k}-(-1)^{d}}{v_{j}+v_{k}} \prod_{i \neq k} \frac{v_{k}+v_{i}}{v_{k}-v_{i}}=1+\frac{1}{2 v_{j}}
$$

for $1 \leq j \leq d$. Let $f(z)=\frac{2 z-(-1)^{d}}{2 z\left(z+v_{j}\right)} \prod_{i=1}^{d} \frac{z+v_{i}}{z-v_{i}}$ and view $f(z)$ as an element of the function field of the projective line defined over $F\left(v_{1}, \ldots, v_{d}\right)$. Then, the left hand side can be interpreted as the sum $\sum_{k=1}^{d} \operatorname{Res}_{z=v_{k}} f(z) d z$, 
where $\operatorname{Res}_{z=v} f(z) d z$ is the residue of $f(z)$ at $v$, if $v \neq \infty$, and it is the residue of $-\frac{1}{z^{2}} f\left(\frac{1}{z}\right)$ at 0 , if $v=\infty$. Thus, the residue theorem for complete non-singular curves implies that

$$
\sum_{k=1}^{d} \frac{2 v_{k}-(-1)^{d}}{v_{j}+v_{k}} \prod_{i \neq k} \frac{v_{k}+v_{i}}{v_{k}-v_{i}}=-\left(\operatorname{Res}_{z=\infty} f(z) d z+\operatorname{Res}_{z=0} f(z) d z\right)=1+\frac{1}{2 v_{j}},
$$

as required. Hence, we have shown that, for $1 \leq j \leq d$,

$$
\gamma_{j}=\left(2 v_{j}-(-1)^{d}\right) \prod_{k \neq j} \frac{v_{j}+v_{k}}{v_{j}-v_{k}}
$$

so (b) is proved. (For a combinatorial proof see Proposition 4.21(a) below.) Now, since $E m=\omega_{0} m$ and $m=\sum_{i=1}^{d} m_{i}$, we have that $\omega_{0}=\sum_{i=1}^{d} \gamma_{i}$. Similarly, we have that $\omega_{a}=\sum_{j=1}^{m} v_{j}^{a} \gamma_{j}$ because

$$
\begin{aligned}
\omega_{a} m & =\frac{\omega_{a}}{\omega_{0}} E m=\frac{1}{\omega_{0}} E X_{1}^{a} E m=E X_{1}^{a} m \\
& =\sum_{i=1}^{d} E X_{1}^{a} m_{i}=\sum_{i=1}^{d} v_{i}^{a} E m_{i}=\left(\sum_{i=1}^{d} v_{i}^{a} \gamma_{i}\right) m .
\end{aligned}
$$

We now show that

$$
\omega_{0}=\sum_{i=1}^{d} \gamma_{i}= \begin{cases}2\left(v_{1}+\cdots+v_{d}\right), & \text { if } d \text { is even } \\ 2\left(v_{1}+\cdots+v_{d}\right)+1, & \text { if } d \text { is odd }\end{cases}
$$

To evaluate $\sum_{i=1}^{d} \gamma_{i}$, we consider $g(z)=\frac{2 z-(-1)^{d}}{2 z} \prod_{i=1}^{d} \frac{z+v_{i}}{z-v_{i}}$ and interpret the sum as $\sum_{i=1}^{d} \operatorname{Res}_{z=v_{i}} g(z) d z$. Then the residue theorem gives the desired formula for $\omega_{0}$.

We next show that $M$ is uniquely determined, up to isomorphism. Suppose that $\mathscr{W}_{r, n}(\mathbf{u})$ has another irreducible module of dimension $d^{\prime}$ upon which $e$ acts non-trivially. Then, by the argument above,

$$
\omega_{0}= \begin{cases}2\left(v_{1}^{\prime}+\cdots+v_{d^{\prime}}^{\prime}\right), & \text { if } d^{\prime} \text { is even, } \\ 2\left(v_{1}^{\prime}+\cdots+v_{d^{\prime}}^{\prime}\right)+1, & \text { if } d^{\prime} \text { is odd }\end{cases}
$$

for some $v_{1}^{\prime}, \ldots, v_{d^{\prime}}^{\prime} \subseteq\left\{u_{1}, \ldots, u_{r}\right\}$. As we are assuming that $u_{1}, \ldots, u_{r}$ are algebraically independent, this forces $d^{\prime}=d$ and $v_{i}^{\prime}=v_{(i) \sigma}$, for some $\sigma \in \mathfrak{S}_{d}$ and $1 \leq i \leq d$. Hence, by (a)-(c), $M \cong M^{\prime}$ as required. 
Finally, it remains to verify that (a)-(c) define a representation of $\mathscr{W}_{r, 2}(\mathbf{u})$ whenever $\omega_{a}=\sum_{i=1}^{d} v_{i}^{a} \gamma_{i}$, for $a \geq 0$ and $\gamma_{i}$ as above. It is easy to check that the action respects the relations $E\left(X_{1}+X_{2}\right)=0=\left(X_{1}+X_{2}\right) E$, $E X_{1}^{a} E=\omega_{a} E$ and $X_{1} S-S X_{2}=E-1=S X_{1}-X_{2} S$. That $S E=E$ and $E S=E$ on $M$, follows from the identity $\sum_{k=1}^{d} \frac{\gamma_{k}}{v_{j}+v_{k}}=1+\frac{1}{2 v_{j}}$ proved above. We now prove that $S^{2}=1$. Observe that $S^{2}$ commutes with $X_{1}$ when acting on $M$. As the $v_{i}$ are pairwise distinct, we have $S^{2} m_{i}=c_{i} m_{i}$, for some $c_{i} \in R$. Explicit computation shows that $c_{i}=\frac{1-2 \gamma_{i}}{4 v_{i}^{2}}+\gamma_{i} \sum_{j=1}^{d} \frac{\gamma_{j}}{\left(v_{i}+v_{j}\right)^{2}}$. Computing the residues of $h(z) d z$, when $h(z)=\frac{2 z-(-1)^{d}}{2 z\left(z+v_{i}\right)^{2}} \prod_{k=1}^{d} \frac{z+v_{i}}{z-v_{i}}$, proves that $c_{i}=1$, for $1 \leq i \leq d$.

Remark 3.3. The action of $X_{1}$ on an irreducible $\mathscr{W}_{r, 2}(\mathbf{u})$-module is not semisimple in general. For example, let $\Omega$ be given by $\omega_{0}=1, \omega_{1}=0$ and

$$
\omega_{a+2}=\frac{1}{2} \omega_{a+1}-\frac{1}{16} \omega_{a}
$$

for $a \geq 0$. For $r=2$ we set $\left(u_{1}, u_{2}\right)=\left(\frac{1}{4}, \frac{1}{4}\right)$ and for $r=3$ set $\left(u_{1}, u_{2}, u_{3}\right)=$ $\left(\frac{1}{4}, \frac{1}{4},-\frac{1}{2}\right)$. Then $\mathscr{W}_{r, 2}(\mathbf{u})$ has a two dimensional irreducible module upon which the generators act as follows:

$$
E \mapsto\left(\begin{array}{cc}
1 & 0 \\
0 & 0
\end{array}\right), \quad S \mapsto\left(\begin{array}{cc}
1 & 0 \\
0 & -1
\end{array}\right), \quad X_{1} \mapsto\left(\begin{array}{cc}
0 & \frac{1}{4} \\
-\frac{1}{4} & \frac{1}{2}
\end{array}\right) \quad \text { and } \quad X_{2} \mapsto\left(\begin{array}{cc}
0 & -\frac{1}{4} \\
\frac{1}{4} & -\frac{1}{2}
\end{array}\right)
$$

Further, $X_{1}-\frac{1}{4} \neq 0$ and $\left(X_{1}-\frac{1}{4}\right)^{2}=0$.

ThEOREM 3.4. Let $F$ be a field in which 2 is invertible and that $u_{1}, \ldots, u_{r}$ are algebraically independent over $F$. Let $R=F\left(u_{1}, \ldots, u_{r}\right)$ and suppose that $\mathscr{W}_{r, 2}(\mathbf{u})$ is a split semisimple $R$-algebra and that $\omega_{0} \neq 0$. Then $\mathscr{W}_{r, 2}(\mathbf{u})$ has dimension $3 r^{2}=\left.r^{n}(2 n-1) ! !\right|_{n=2}$ if and only if $\mathscr{W}_{r, 2}(\mathbf{u})$ has an irreducible representation of dimension $r$ upon which $E$ acts non-trivially.

Proof. We have constructed all the irreducible $\mathscr{W}_{r, 2}$-modules in Propositions 3.1 and 3.2 above. Under our assumptions, Proposition 3.1 implies that $\mathscr{W}_{r, 2}(\mathbf{u})$ has (a) $2 r$ pairwise non-isomorphic one dimensional representations and (b) $\left(\begin{array}{l}r \\ 2\end{array}\right)$ pairwise non-isomorphic two dimensional representations. Note that case (c) from Proposition 3.1 does not occur since $u_{1}, \ldots, u_{r}$ are pairwise distinct. Further, Proposition 3.2 implies that $\mathscr{W}_{r, 2}(\mathbf{u})$ has a unique irreducible representation $M$ such that $E M \neq 0$ and, moreover, if 
$d=\operatorname{dim} M$ then $1 \leq d \leq r$. Hence, by the Wedderburn-Artin theorem we have

$$
\operatorname{dim} \mathscr{W}_{r, 2}(\mathbf{u})=2 r+4\left(\begin{array}{l}
r \\
2
\end{array}\right)+d^{2}=2 r^{2}+d^{2},
$$

so that $\operatorname{dim} \mathscr{W}_{r, 2}(\mathbf{u})=3 r^{2}$ if and only if $r=d$. The result follows.

Note that by Proposition 3.2, under the conditions of the theorem, $\mathscr{W}_{r, 2}(\mathbf{u})$ has a (unique) representation of dimension $r$ upon which $E$ acts non-trivially if and only if $\omega_{a}=\sum_{j=1}^{r} u_{j}^{a} \gamma_{j}$, for $a \geq 0$, where $\gamma_{i}=\left(2 u_{i}-\right.$ $\left.(-1)^{r}\right) \prod_{\substack{1 \leq j \leq r \\ j \neq i}} \frac{u_{i}+u_{j}}{u_{i}-u_{j}}$.

Recall that Schur's $q$-functions $q_{a}=q_{a}(\mathbf{x})$ in the indeterminates $\mathbf{x}=$ $\left(x_{1}, \ldots, x_{r}\right)[$ Mac95, p. 250] are defined by the equation

$$
\prod_{i=1}^{r} \frac{1+x_{i} y}{1-x_{i} y}=\sum_{a \geq 0} q_{a}(\mathbf{x}) y^{a} .
$$

Note that $q_{a}(\mathbf{x})$ is a polynomial in $\mathbf{x}$, for all $a \geq 0$.

Lemma 3.5. Assume that $R$ is an integral domain and that 2 is invertible in $R$. Suppose that $\mathbf{u} \in R^{r}$, with $u_{i}-u_{j} \neq 0$ whenever $i \neq j$. Let $F$ be the quotient field of $R$ and for $a \geq 0$ define

$$
\omega_{a}=\sum_{i=1}^{r}\left(2 u_{i}-(-1)^{r}\right) u_{i}^{a} \prod_{\substack{1 \leq j \leq r \\ j \neq i}} \frac{u_{i}+u_{j}}{u_{i}-u_{j}} \in F,
$$

as in Theorem 3.4. Then $\omega_{a}=q_{a+1}(\mathbf{u})-\frac{1}{2}(-1)^{r} q_{a}(\mathbf{u})+\frac{1}{2} \delta_{a 0}$. In particular, $\omega_{a} \in R$.

Proof. If $a=0$ then the result follows from Proposition 3.2, so we can assume that $a>0$. Let $f(z)=\frac{1}{2} z^{a-1}\left(2 z-(-1)^{r}\right) \prod_{i=1}^{r} \frac{z+u_{i}}{z-u_{i}}$. Then $\omega_{a}$ can be interpreted as $\sum_{i=1}^{r} \operatorname{Res}_{z=u_{i}} f(z) d z=-\operatorname{Res}_{z=\infty} f(z) d z$. Calculating the residue of $f(z) d z$ at $z=\infty$ now shows that $\omega_{a}=q_{a+1}(\mathbf{u})-$ $\frac{1}{2}(-1)^{r} q_{a}(\mathbf{u})+\frac{1}{2} \delta_{a 0}$. (See [Mac95, (2.9), p. 209] for a more direct proof.) Hence, $\omega_{a} \in R$ since $q_{b}(\mathbf{x}) \in R[\mathbf{x}]$, for $b \geq 0$.

We want the cyclotomic Nazarov-Wenzl algebras to be "cyclotomic" generalizations of the Brauer algebras. In particular, we want them to be free $R$-modules of rank $r^{n}(2 n-1)$ !!. Theorem 3.4 gives sufficient conditions 
on $\Omega=\left\{\omega_{a} \mid a \geq 0\right\}$ for $\mathscr{W}_{r, 2}(\mathbf{u})$ to have dimension $r^{n}(2 n-1)$ !! when $R$ is an algebraically closed field and $n=2$. Consequently, in our study of $\mathscr{W}_{r, n}(\mathbf{u})$ we will require that $\Omega$ have the following property.

Definition 3.6. Let $\Omega=\left\{\omega_{a} \mid a \geq 0\right\} \subseteq R$ and suppose that $\mathbf{u} \in R^{r}$. Then $\Omega$ is $\mathbf{u}$-admissible if $\omega_{a}=q_{a+1}(\mathbf{u})-\frac{1}{2}(-1)^{r} q_{a}(\mathbf{u})+\frac{1}{2} \delta_{a 0}$, for $a \geq 0$.

Remark 3.7. Let $R=\mathbb{Z}[\mathbf{u}]$ where $u_{1}, \ldots, u_{r}$ are indeterminates. Assume that each $\omega_{a}$, for $a \geq 0$, is a polynomial in $\mathbf{u}$ and that $\omega_{0} \neq 0$. Then, by Theorem 3.4 and Theorems 5.3 and 7.17 below, $\Omega$ is $\mathbf{u}$-admissible if and only if

a) $\mathscr{W}_{r, n}(\Omega) \otimes_{\mathbb{Z}[\mathbf{u}]} \mathbb{Q}(\mathbf{u})$ is semisimple, and,

b) $\mathscr{W}_{r, n}(\Omega)$ is a free $R$-module of rank $r^{n}(2 n-1) !$ !,

for all $n \geq 0$.

Recall from Remark 2.11 that $\widetilde{W}_{1}(y)=\sum_{a \geq 0} \omega_{a} y^{-a}$, where $y$ is an indeterminate.

Lemma 3.8. Suppose that $\mathbf{u} \in R^{r}$. Then $\Omega$ is $\mathbf{u}$-admissible if and only if

$$
\widetilde{W}_{1}(y)+y-\frac{1}{2}=\left(y-\frac{1}{2}(-1)^{r}\right) \prod_{i=1}^{r} \frac{y+u_{i}}{y-u_{i}} .
$$

Proof. By definition, $\mathfrak{u}$-admissibility is equivalent to the identity

$$
\widetilde{W}_{1}(y)=\frac{1}{2}+\sum_{a \geq 0}\left(q_{a+1}(\mathbf{u})-\frac{1}{2}(-1)^{r} q_{a}(\mathbf{u})\right) y^{-a} .
$$

Now expand this equation using the definition of the Schur $q$-functions.

Corollary 3.9. Suppose that $\Omega$ is $\mathbf{u}$-admissible. Then $\Omega$ is admissible.

Proof. First suppose that $\mathbf{x}=\left(x_{1}, \ldots, x_{r}\right)$ are algebraically independent and let $\Omega=\left\{\omega_{a} \mid a \geq 0\right\}$, where $\omega_{a}=q_{a+1}(\mathbf{x})-\frac{1}{2}(-1)^{r} q_{a}(\mathbf{x})+\frac{1}{2} \delta_{a 0}$, for $a \geq 0$. Then $\Omega$ is $\mathbf{x}$-admissible by definition and hence admissible by Corollary 2.4 and Proposition 3.2. Therefore, by the definition of admissibility we have the following polynomial identity in $x_{1}, \ldots, x_{r}$

$$
\omega_{2 a+1}=\frac{1}{2}\left\{-\omega_{2 a}+\sum_{b=1}^{2 a+1}(-1)^{b-1} \omega_{b-1} \omega_{2 a+1-b}\right\} .
$$


The general case now follows by specializing $x_{i}=u_{i}$, for $1 \leq i \leq r$.

For a second proof, note that if $\Omega$ is $\mathbf{u}$-admissible then

$$
\left(\widetilde{W}_{1}(y)+y-\frac{1}{2}\right)\left(\widetilde{W}_{1}(-y)-y-\frac{1}{2}\right)=\left(\frac{1}{2}-y\right)\left(\frac{1}{2}+y\right),
$$

by Lemma 3.8. Hence, $\Omega$ is admissible by Remark 2.11.

DEFinition 3.10. The parameter set $\Omega$ is rational if there exists a $k>0$ and $a_{1}, \ldots, a_{k} \in R$ such that $\Omega$ satisfies the linear recursion

$$
\omega_{i+k}+a_{1} \omega_{i+k-1}+\cdots+a_{k} \omega_{i}=0,
$$

for all $i \gg 0$.

Equivalently, $\Omega$ is rational if $\Omega$ is admissible and $\widetilde{W}_{1}(y)$ is a rational function. See Lemma 7.18 for another characterization of rationality.

Rationality allows us to give a partial converse to Corollary 3.9.

Proposition 3.11. Suppose that $R$ is an algebraically closed field and that $\Omega$ is rational. Then every finite dimensional irreducible $\mathscr{W}_{n}^{\text {aff }}(\Omega)$-module can be considered as an irreducible module for some cyclotomic NazarovWenzl algebra $\mathscr{W}_{r, n}(\mathbf{u})$ with $\Omega$ being $\mathbf{u}$-admissible.

In particular, if $\Omega$ is rational then $\Omega$ is $\mathbf{u}$-admissible for some $\mathbf{u}$.

Proof. As $\Omega$ is rational, $\widetilde{W}_{1}(y)$ is a rational function and we may write

$$
\frac{\widetilde{W}_{1}(y)+y-\frac{1}{2}}{y+\frac{1}{2}}=\frac{\prod_{i}\left(y-\alpha_{i}\right)^{n_{i}}}{\prod_{j}\left(y-\beta_{j}\right)^{m_{j}}},
$$

for some non-negative integers $n_{i}$ and $m_{j}$ and with the $\alpha_{i}, \beta_{j} \in R$ being pairwise distinct. Using Remark 2.11 it follows easily that

$$
\widetilde{W}_{1}(y)+y-\frac{1}{2}=\left(y+\frac{1}{2}\right) \prod_{i=1}^{s} \frac{y+c_{i}}{y-c_{i}},
$$

for some $c_{i} \in R$ and some $s \geq 0$.

Now suppose that $M$ is a finite dimensional irreducible $\mathscr{W}_{n}^{\text {aff }}(\Omega)$-module and let $\left(X_{1}-\lambda_{1}\right) \cdots\left(X_{1}-\lambda_{d}\right)$ be the characteristic polynomial for the action of $X_{1}$ on $M$. Set

$$
\mathbf{u}= \begin{cases}\left(c_{1}, \ldots, c_{s}, \lambda_{1}, \ldots, \lambda_{d},-\lambda_{1}, \ldots,-\lambda_{d}\right), & \text { if } s \text { is odd } \\ \left(c_{1}, \ldots, c_{s}, \lambda_{1}, \ldots, \lambda_{d},-\lambda_{1}, \ldots,-\lambda_{d}, 0\right), & \text { if } s \text { is even }\end{cases}
$$

Put $r=s+2 d$ if $s$ is odd and $r=s+2 d+1$ if $s$ is even. Then $M$ is an irreducible $\mathscr{W}_{r, n}(\mathbf{u})$-module and $\Omega$ is $\mathbf{u}$-admissible. 
We will improve on this result by showing that we can construct all of the irreducible modules for the affine Wenzl algebras in Theorem 7.19 below.

\section{$\S 4$. The seminormal representations of $\mathscr{W}_{r, n}(\mathbf{u})$}

In this section, we will give an explicit description of the irreducible representations of $\mathscr{W}_{r, n}(\mathbf{u})$ in the special case when $R$ is an field of characteristic greater than $2 n$ and when the parameters $\mathbf{u}$ satisfy some rather technical assumptions; see Theorem 4.13.

The semisimple irreducible representations of the Brauer algebra $\mathscr{B}_{n}(\omega)$ are labelled by partitions of $n-2 m$, where $0 \leq m \leq\left\lfloor\frac{n}{2}\right\rfloor$, and a basis of the representation indexed by the partition $\lambda$ is indexed by the set of updown $\lambda$-tableaux. Analogously, we might expect that the semisimple irreducible representations of $\mathscr{W}_{r, n}(\mathbf{u})$ should be indexed by the multipartitions of $n-2 m$, with the bases of these modules being indexed by the updown $\lambda$-tableaux, where $\lambda$ is a multipartition. We will see that this is the case. We begin by defining these combinatorial objects.

Recall that a partition of $m$ is a sequence of weakly decreasing nonnegative integers $\tau=\left(\tau_{1}, \tau_{2}, \ldots\right)$ such that $|\tau|:=\tau_{1}+\tau_{2}+\cdots=m$. Similarly, an $r$-multipartition of $m$, or more simply a multipartition, is an ordered $r$-tuple $\lambda=\left(\lambda^{(1)}, \ldots, \lambda^{(r)}\right)$ of partitions $\lambda^{(s)}$, with $|\lambda|:=\left|\lambda^{(1)}\right|+$ $\cdots+\left|\lambda^{(r)}\right|=m$. If $\lambda$ is a multipartition of $m$ then we write $\lambda \vdash m$.

If $\lambda$ and $\mu$ are two multipartitions we say that $\mu$ is obtained from $\lambda$ by adding a box if there exists a pair $(i, s)$ such that $\mu_{i}^{(s)}=\lambda_{i}^{(s)}+1$ and $\mu_{j}^{(t)}=$ $\lambda_{j}^{(t)}$ for $(j, t) \neq(i, s)$. In this situation we will also say that $\lambda$ is obtained from $\mu$ by removing a box and we write $\lambda \subset \mu$ and $\mu \backslash \lambda=\left(i, \lambda_{i}^{(s)}, s\right)$. We will also say that the triple $\left(i, \lambda_{i}^{(s)}, s\right)$ is an addable node of $\lambda$ and a removable node of $\mu$. Note that $|\mu|=|\lambda|+1$.

Fix an integer $m$ with $0 \leq m \leq\left\lfloor\frac{n}{2}\right\rfloor$ and let $\lambda$ be a multipartition of $n-2 m$. An $n$-updown $\lambda$-tableau, or more simply an updown $\lambda$-tableau, is a sequence $\mathfrak{u}=\left(\mathfrak{u}_{1}, \mathfrak{u}_{2}, \ldots, \mathfrak{u}_{n}\right)$ of multipartitions where $\mathfrak{u}_{n}=\lambda$ and the multipartition $\mathfrak{u}_{i}$ is obtained from $\mathfrak{u}_{i-1}$ by either adding or removing a box, for $i=1, \ldots, n$, where we set $\mathfrak{u}_{0}$ equal to the empty multipartition $\emptyset$. Let $\mathscr{T}_{n}^{u d}(\lambda)$ be the set of updown $\lambda$-tableaux of $n$. Note that $\lambda$ is a multipartition of $n-2 m$ and each element of $\mathscr{T}_{n}^{u d}(\lambda)$ is an $n$-tuple of multipartitions, so the $n$ is necessary in this notation. 
In the special case when $\lambda$ is a multipartition of $n$ (so $m=0$ ), there is a natural bijection between the set of $n$-updown $\lambda$-tableaux and the set of standard $\lambda$-tableaux in the sense of [DJM99]. This is the origin of the terminology of updown $\lambda$-tableaux. If $\lambda$ is a multipartition of $n$ we set $\mathscr{T}^{\text {std }}(\lambda)=\mathscr{T}_{n}^{u d}(\lambda)$ and refer to the elements of $\mathscr{T}^{\text {std }}(\lambda)$ as standard $\lambda$ tableaux.

Definition 4.1. Suppose $1 \leq k \leq n$. Define an equivalence relation $\stackrel{k}{\sim}$ on $\mathscr{T}_{n}^{u d}(\lambda)$ by declaring that $\mathfrak{u} \stackrel{k}{\sim} \mathfrak{t}$ if $\mathfrak{u}_{j}=\mathfrak{t}_{j}$ whenever $1 \leq j \leq n$ and $j \neq k$, for $\mathfrak{t}, \mathfrak{u} \in \mathscr{T}_{n}^{u d}(\lambda)$.

The following result is an immediate consequence of Definition 4.1.

Lemma 4.2. Suppose $\mathfrak{t} \in \mathscr{T}_{n}^{u d}(\lambda)$ with $\mathfrak{t}_{k-1}=\mathfrak{t}_{k+1}$. Then there is a bijection between the set of all addable and removable nodes of $\mathfrak{t}_{k-1}$ and the set of $\mathfrak{u} \in \mathscr{T}_{n}^{u d}(\lambda)$ with $\mathfrak{u} \stackrel{k}{\sim} \mathfrak{t}$.

Let $\lambda$ be a multipartition and suppose that $\mathfrak{u}$ is an $n$-updown $\lambda$-tableaux. For $k=2, \ldots, n$ the multipartitions $\mathfrak{u}_{k}$ and $\mathfrak{u}_{k-1}$ differ by exactly one box; so either $\mathfrak{u}_{k} \subset \mathfrak{u}_{k-1}$ or $\mathfrak{u}_{k-1} \subset \mathfrak{u}_{k}$. We define the content of $k$ in $\mathfrak{u}$ to be the scalar $c_{\mathfrak{u}}(k) \in R$ given by

$$
c_{\mathfrak{u}}(k)= \begin{cases}j-i+u_{s}, & \text { if } \mathfrak{u}_{k} \backslash \mathfrak{u}_{k-1}=(i, j, s), \\ i-j-u_{s}, & \text { if } \mathfrak{u}_{k-1} \backslash \mathfrak{u}_{k}=(i, j, s)\end{cases}
$$

More generally, if $\alpha=(i, j, s)$ is an addable node of $\lambda$ we define $c(\alpha)=$ $u_{s}+j-i$ and if $\alpha$ is a removable node of $\lambda$ we set $c(\alpha)=-\left(u_{s}+j-i\right)$.

The key property of contents that we need to construct the seminormal representations is the following. Note that we are not (yet) assuming that $R$ is a field.

Definition 4.3. The parameters $\mathbf{u}=\left(u_{1}, \ldots, u_{r}\right)$ are generic for $\mathscr{W}_{r, n}(\mathbf{u})$ if whenever there exists $d \in \mathbb{Z}$ such that either $u_{i} \pm u_{j}=d \cdot 1_{R}$ and $i \neq j$, or $2 u_{i}=d \cdot 1_{R}$ then $|d| \geq 2 n$.

For example, $\mathbf{u}$ is generic for $\mathscr{W}_{r, n}(\mathbf{u})$ if $u_{1}, \ldots, u_{r}$ are algebraically independent over a subfield of $R$.

LEMmA 4.4. Suppose that the parameters $\mathbf{u}$ are generic for $\mathscr{W}_{r, n}(\mathbf{u})$ and that char $R>2 n$. Let $\lambda$ be a multipartition of $n-2 m$, where $0 \leq m \leq$ $\left\lfloor\frac{n}{2}\right\rfloor$, and suppose that $\mathfrak{t}, \mathfrak{u} \in \mathscr{T}_{n}^{u d}(\lambda)$. Then 
a) $\mathfrak{t}=\mathfrak{u}$ if and only if $c_{\mathfrak{t}}(k)=c_{\mathfrak{u}}(k)$, for $k=1, \ldots, n$;

b) if $1 \leq k<n$ then $c_{\mathfrak{t}}(k)-c_{\mathfrak{t}}(k+1) \neq 0$; and,

c) if $\mathfrak{t}_{k-1}=\mathfrak{t}_{k+1}$ then $c_{\mathfrak{t}}(k) \pm c_{\mathfrak{u}}(k) \neq 0$, whenever $\mathfrak{u} \stackrel{k}{\sim} \mathfrak{t}$ and $\mathfrak{u} \neq \mathfrak{t}$.

d) $2 c_{\mathfrak{t}}(k) \pm 1 \neq 0$, for $1 \leq k \leq n$.

Proof. Part (a) follows by induction on $n$. The key point is that our assumptions imply that the contents of the addable and removable nodes in $\lambda$ are distinct so a $\lambda$-tableau $\mathfrak{t}$ is uniquely determined by the sequence of contents $c_{\mathfrak{t}}(k)$, for $k=1, \ldots, n$. The same argument proves parts (b), (c) and $(\mathrm{d})$.

Until further notice we fix an integer $m$ with $0 \leq m \leq\left\lfloor\frac{n}{2}\right\rfloor$ and we fix a multipartition $\lambda$ of $n-2 m$.

Motivated by [Naz96], we introduce the following rational functions in an indeterminate $y$. These functions will play a key role in the construction of seminormal representations of $\mathscr{W}_{r, n}(\mathbf{u})$.

Definition 4.5. Suppose that $\mathfrak{t} \in \mathscr{T}_{n}^{u d}(\lambda)$. For $1 \leq k \leq n$, define rational functions $W_{k}(y, \mathfrak{t})$ by

$$
W_{k}(y, \mathfrak{t})=\frac{1}{2}-y+\left(y-\frac{1}{2}(-1)^{r}\right) \prod_{\alpha} \frac{y+c(\alpha)}{y-c(\alpha)}
$$

where $\alpha$ runs over the addable and removable nodes of the multipartition $\mathfrak{t}_{k-1}$.

The rational functions $W_{k}(y, \mathfrak{t})$ are related to the combinatorics above by the following result. If $f(y)$ is a rational function and $\alpha \in R$ then we write $\operatorname{Res}_{y=\alpha} f(y)$ for the residue of $f(y)$ at $y=\alpha$.

LEMmA 4.6. Suppose that $\mathbf{u}$ is generic and char $R>2 n$. Let $\mathfrak{t} \in$ $\mathscr{T}_{n}^{u d}(\lambda)$ and $1 \leq k \leq n$. Then

$$
\frac{W_{k}(y, \mathfrak{t})}{y}=\sum_{\alpha}\left(\operatorname{Res}_{y=c(\alpha)} \frac{W_{k}(y, \mathfrak{t})}{y}\right) \cdot \frac{1}{y-c(\alpha)}
$$

where $\alpha$ runs over the addable and removable nodes of $\mathfrak{t}_{k-1}$. 
Proof. As the $c(\alpha)$ are pairwise distinct, we can certainly write

$$
\frac{W_{k}(y, \mathfrak{t})}{y}=a+\frac{b}{y}+\sum_{\alpha}\left(\operatorname{Res}_{y=c(\alpha)} \frac{W_{k}(y, \mathfrak{t})}{y}\right) \cdot \frac{1}{y-c(\alpha)}
$$

for some $a, b \in R$, where $\alpha$ runs over the addable and removable nodes of $\mathfrak{t}_{k-1}$. Now, $a=\left.\frac{W_{k}(y, \mathfrak{t})}{y}\right|_{y=\infty}=0$. Let $c$ be the number of addable and removable nodes of $\mathfrak{t}_{k-1}$. Since a partition always has an odd number of addable and removable nodes, we have that $(-1)^{c}=(-1)^{r}$. Therefore,

$$
b=\operatorname{Res}_{y=0} \frac{W_{k}(y, \mathfrak{t})}{y}=\frac{1}{2}\left(1-(-1)^{c}(-1)^{r}\right)=0,
$$

as we needed to show.

We are now ready to define the matrices which make up the seminormal form.

Definition 4.7. Let $\lambda$ be a multipartition and $k$ an integer with $1 \leq$ $k \leq n$. Suppose that $\mathfrak{t}$ and $\mathfrak{u}$ are updown $\lambda$-tableaux in $\mathscr{T}_{n}^{u d}(\lambda)$ such that $\mathfrak{t}_{k-1}=\mathfrak{t}_{k+1}$. Then we define the scalars $e_{\mathfrak{t u}}(k) \in R$ by

$$
e_{\mathfrak{t} \mathfrak{u}}(k)= \begin{cases}\operatorname{Res}_{y=c_{\mathfrak{t}}(k)} \frac{W_{k}(y, \mathfrak{t})}{y}, & \text { if } \mathfrak{t}=\mathfrak{u}, \\ \sqrt{e_{\mathfrak{t} \mathfrak{t}}(k)} \sqrt{e_{\mathfrak{u} \mathfrak{u}}(k)}, & \text { if } \mathfrak{t} \neq \mathfrak{u} \text { and } \mathfrak{u} \stackrel{k}{\sim} \mathfrak{t} \\ 0, & \text { otherwise. }\end{cases}
$$

(In (4.12) below we will fix the choice of square roots $\sqrt{e_{\mathfrak{t t}}(k)}$, for $\mathfrak{t} \in \mathscr{T}_{n}^{u d}(\lambda)$ and $1 \leq k \leq n$.)

Note that when $c_{\mathfrak{t}}(k) \neq 0$ then $e_{\mathfrak{t t}}(k)=\operatorname{Res}_{y=c_{\mathfrak{t}}(k)} \frac{W_{k}(y, \mathfrak{t})+y-1 / 2}{y}$.

We remark that if $\mathfrak{t}_{k-1} \neq \mathfrak{t}_{k+1}$ then the definition of $e_{\mathfrak{t}}(k)$ still makes sense, however, we do not define $e_{\mathfrak{t t}}(k)$ in this case as we will not need it (see Theorem 4.13 below).

It follows from Definition 4.5 that

$$
e_{\mathfrak{t} \mathfrak{t}}(k)=\left(2 c_{\mathfrak{t}}(k)-(-1)^{r}\right) \prod_{\alpha} \frac{c_{\mathfrak{t}}(k)+c(\alpha)}{c_{\mathfrak{t}}(k)-c(\alpha)}
$$


where $\alpha$ runs over all addable and removable nodes of $\mathfrak{t}_{k-1}$ with $c(\alpha) \neq c_{\mathfrak{t}}(k)$. Note that Lemma 4.4 now implies that:

$$
\text { If } \mathfrak{u} \stackrel{k}{\sim} \mathfrak{t} \text { then } e_{\mathfrak{t u}}(k) \neq 0 \text {, for } 1 \leq k<n .
$$

This will be used many times below without mention. Also observe that Lemma 4.6 can be restated as

$$
\frac{W_{k}(y, \mathfrak{t})}{y}=\sum_{\substack{k \\ \mathfrak{u} \sim \mathfrak{t}}} \frac{e_{\mathfrak{u} \mathfrak{u}}(k)}{y-c_{\mathfrak{u}}(k)}
$$

Given two partitions $\mathfrak{t}$ and $\mathfrak{u}$ write $\mathfrak{t} \ominus \mathfrak{u}=\alpha$ if either $\mathfrak{u} \subset \mathfrak{t}$ and $\mathfrak{t} \backslash \mathfrak{u}=\alpha$, or $\mathfrak{t} \subset \mathfrak{u}$ and $\mathfrak{u} \backslash \mathfrak{t}=\alpha$.

Definition 4.10. Let $\mathfrak{t} \in \mathscr{T}_{n}^{u d}(\lambda)$ and suppose that $\mathfrak{t}_{k-1} \neq \mathfrak{t}_{k+1}$, for some $k$ with $1 \leq k<n$.

a) We define

$$
a_{\mathfrak{t}}(k)=\frac{1}{c_{\mathfrak{t}}(k+1)-c_{\mathfrak{t}}(k)} \quad \text { and } \quad b_{\mathfrak{t}}(k)=\sqrt{1-a_{\mathfrak{t}}(k)^{2}} .
$$

(We fix the choice of square root for $b_{\mathfrak{t}}(k)$ in $(4.12)$ below. Note that $c_{\mathfrak{t}}(k+1)-c_{\mathfrak{t}}(k) \neq 0$ by Lemma $4.4(\mathrm{~b})$.)

b) If $\mathfrak{t}_{k} \ominus \mathfrak{t}_{k-1}$ and $\mathfrak{t}_{k+1} \ominus \mathfrak{t}_{k}$ are in different rows and in different columns then we define $S_{k} \mathfrak{t}$ to be the updown $\lambda$-tableau

$$
S_{k} \mathfrak{t}=\left(\mathfrak{t}_{1}, \ldots, \mathfrak{t}_{k-1}, \mathfrak{u}_{k}, \mathfrak{t}_{k+1}, \ldots, \mathfrak{t}_{n}\right)
$$

where $\mathfrak{u}_{k}$ is the multipartition which is uniquely determined by the conditions $\mathfrak{u}_{k} \ominus \mathfrak{t}_{k+1}=\mathfrak{t}_{k-1} \ominus \mathfrak{t}_{k}$ and $\mathfrak{t}_{k-1} \ominus \mathfrak{u}_{k}=\mathfrak{t}_{k} \ominus \mathfrak{t}_{k+1}$. If the nodes $\mathfrak{t}_{k} \ominus \mathfrak{t}_{k-1}$ and $\mathfrak{t}_{k+1} \ominus \mathfrak{t}_{k}$ are both in the same row, or both in the same column, then $S_{k} \mathfrak{t}$ is not defined.

We remark that if $\mathfrak{t}_{k-1}=\mathfrak{t}_{k+1}$ then the definitions of $a_{\mathfrak{t}}(k)$ and $b_{\mathfrak{t}}(k)$ both make sense, however, we do not define them in this case as we will never need them (see Theorem 4.13 below). Moreover, the condition $\mathfrak{t}_{k-1} \neq \mathfrak{t}_{k+1}$ is crucial in proving Lemma 4.11(b) below. (In fact, if we drop this condition then Lemma 4.11(b) is not correct.)

We leave the following Lemma as an exercise to help the reader familiarize themselves with the definitions. 
LemmA 4.11. Suppose that $\mathfrak{t} \in \mathscr{T}_{n}^{u d}(\lambda)$ and $1 \leq k<n$. Then:

a) If $S_{k} \mathfrak{t}$ is defined then $c_{\mathfrak{t}}(k)=c_{S_{k}}(k+1)$ and $c_{\mathfrak{t}}(k+1)=c_{S_{k}}(k)$; consequently, $a_{S_{k}}(k)=-a_{\mathfrak{t}}(k)$.

b) If $S_{k} \mathfrak{t}$ is not defined then $a_{\mathfrak{t}}(k)= \pm 1$ and $b_{\mathfrak{t}}(k)=0$.

Finally, if $\mathfrak{t}_{k-1}=\mathfrak{t}_{k+1}$ and $\mathfrak{u} \stackrel{k}{\sim} \mathfrak{t}$, where $1 \leq k<n$, we set

$$
s_{\mathfrak{t} \mathfrak{u}}(k)=\frac{e_{\mathfrak{t} \mathfrak{u}}(k)-\delta_{\mathfrak{t u}}}{c_{\mathfrak{t}}(k)+c_{\mathfrak{u}}(k)} .
$$

Note that $c_{\mathfrak{t}}(k)+c_{\mathfrak{u}}(k) \neq 0$ by Lemma 4.4 .

We will assume that we have chosen the square roots in the definitions of $b_{\mathfrak{t}}(k)$ and $e_{\mathfrak{t} \mathfrak{u}}(k)$ so that the following equalities hold.

Assumption 4.12. (Root conditions) We assume that the ring $R$ is large enough so that $\sqrt{e_{\mathfrak{t t}}(k)} \in R$ and $b_{\mathfrak{t}}(k)=\sqrt{1-a_{\mathfrak{t}}(k)^{2}} \in R$, for all $\mathfrak{t}, \mathfrak{u} \in \mathscr{T}_{n}^{u d}(\lambda)$ and $1 \leq k<n$, and that the following equalities hold:

a) If $\mathfrak{t}_{k-1} \neq \mathfrak{t}_{k+1}$ and $S_{k} \mathfrak{t}$ is defined then $b_{S_{k} \mathfrak{t}}(k)=b_{\mathfrak{t}}(k)$.

b) If $\mathfrak{t}_{k-1} \neq \mathfrak{t}_{k+1}$ and $\mathfrak{t} \stackrel{l}{\sim} \mathfrak{u}$, where $|k-l|>1$, then $b_{\mathfrak{t}}(k)=b_{\mathfrak{u}}(k)$.

c) If $\mathfrak{t}_{k-1} \neq \mathfrak{t}_{k+1}, \mathfrak{t}_{k} \neq \mathfrak{t}_{k+2}$ and $S_{k} \mathfrak{t}$ and $S_{k+1} \mathfrak{t}$ are both defined then $b_{S_{k+1} \mathfrak{t}}(k)=b_{S_{k} \mathfrak{t}}(k+1)$.

d) If $\mathfrak{t}_{k-1}=\mathfrak{t}_{k+1}$ and $\mathfrak{t}_{k}=\mathfrak{t}_{k+2}$ then $\sqrt{e_{\mathfrak{t t}}(k)} \sqrt{e_{\mathfrak{t} \mathfrak{t}}(k+1)}=1$.

e) If $\mathfrak{t}_{k-1}=\mathfrak{t}_{k+1}, \mathfrak{u}_{k-1}=\mathfrak{u}_{k+1}$ and $e_{\mathfrak{t t}}(k)=e_{\mathfrak{u u}}(k)$ then $\sqrt{e_{\mathfrak{t t}}(k)}=$ $\sqrt{e_{\mathfrak{u u}}(k)}$.

f) If $\mathfrak{t}_{k-1}=\mathfrak{t}_{k+1}, \mathfrak{t}_{k}=\mathfrak{t}_{k+2}$ and $\mathfrak{u} \stackrel{k+1}{\sim} \mathfrak{t}, \mathfrak{w} \stackrel{k}{\sim} \mathfrak{t}$ with $S_{k} \mathfrak{u}$ and $S_{k+1} \mathfrak{w}$ both defined and $S_{k} \mathfrak{u}=S_{k+1} \mathfrak{w}$ then $b_{\mathfrak{u}}(k) \sqrt{e_{\mathfrak{u}}(k+1)}=b_{\mathfrak{w}}(k+$ 1) $\sqrt{e_{\mathfrak{w w}}(k)}$.

In Lemma 5.4 below we show that if $R=\mathbb{R}$ then it is possible to choose $\mathbf{u}$ so that the Root Condition is satisfied.

Assuming (4.12) we can now give the formulas for the seminormal representations of $\mathscr{W}_{r, n}(\mathbf{u})$.

THEOREM 4.13. Suppose that $R$ is a field such that char $R>2 n$ and that the root conditions (4.12) hold in $R$. Assume that $\mathbf{u}$ is generic for 
$\mathscr{W}_{r, n}(\mathbf{u})$. Let $\Delta(\lambda)$ be the $R$-vector space with basis $\left\{v_{\mathfrak{t}} \mid \mathfrak{t} \in \mathscr{T}_{n}^{u d}(\lambda)\right\}$. Then $\Delta(\lambda)$ becomes a $\mathscr{W}_{r, n}(\mathbf{u})$-module via

$$
\begin{aligned}
\text { - } S_{k} v_{\mathfrak{t}} & = \begin{cases}\sum_{\mathfrak{k}} s_{\mathfrak{t} \mathfrak{u}}(k) v_{\mathfrak{u}}, & \text { if } \mathfrak{t}_{k-1}=\mathfrak{t}_{k+1}, \\
a_{\mathfrak{t}}(k) v_{\mathfrak{t}}+b_{\mathfrak{t}}(k) v_{S_{k} \mathfrak{t}}, & \text { if } \mathfrak{t}_{k-1} \neq \mathfrak{t}_{k+1},\end{cases} \\
\text { - } E_{k} v_{\mathfrak{t}} & = \begin{cases}\sum_{\mathfrak{k}}^{k} e_{\mathfrak{t} \mathfrak{u}}(k) v_{\mathfrak{u}}, & \text { if } \mathfrak{t}_{k-1}=\mathfrak{t}_{k+1} \\
\mathfrak{u}^{0}, & \text { if } \mathfrak{t}_{k-1} \neq \mathfrak{t}_{k+1},\end{cases} \\
\text { - } X_{j} v_{\mathfrak{t}} & =c_{\mathfrak{t}}(j) v_{\mathfrak{t}},
\end{aligned}
$$

for $1 \leq k<n$ and $1 \leq j \leq n$ and where we set $v_{S_{k} \mathfrak{t}}=0$ if $S_{k} \mathfrak{t}$ is not defined.

DEFinition 4.14. We call $\Delta(\lambda)$ a seminormal representation of $\mathscr{W}_{r, n}(\mathbf{u})$.

We note that the action of the operators $E_{k}$ and $S_{k}$ on $\Delta(\lambda)$, with respect to the basis $\left\{v_{\mathfrak{t}} \mid \mathfrak{t} \in \mathscr{T}_{n}^{u d}(\lambda)\right\}$, are given by symmetric matrices, for $0 \leq k<n$.

For the remainder of this section we assume that $R$ is an algebraically closed field with char $R>2 n$ and that the parameters $\mathbf{u}$ are generic for $\mathscr{W}_{r, n}(\mathbf{u})$ and satisfy (4.12). The proof of Theorem 4.13 will occupy the rest of this section. Our strategy is to use the rational functions $W_{k}(\mathfrak{t}, k)$ to verify that the action that we have just defined of $\mathscr{W}_{r, n}(\mathbf{u})$ on $\Delta(\lambda)$ respects all of the relations in $\mathscr{W}_{r, n}(\mathbf{u})$.

Throughout this section it will be convenient to work with formal (infinite) linear combinations of elements of $\Delta(\lambda)$ and $\mathscr{W}_{r, n}(\mathbf{u})$; alternatively, the reader may prefer to think that we have extended our coefficient ring from $R$ to $R\left(\left(y^{-1}\right)\right)$, where $y$ is an indeterminate over $R$. In fact, at times we will need to work with formal series involving more than one indeterminate.

If $A$ is an algebra we let $Z(A)$ be its center.

LEMmA 4.15. Suppose $k \geq 0$ and that $a \geq 0$. Then there exist elements $\omega_{k}^{(a)}$ in $Z\left(\mathscr{W}_{r, k-1}(\mathbf{u})\right) \cap R\left[X_{1}, \ldots, X_{k-1}\right]$ such that

$$
E_{k} X_{k}^{a} E_{k}=\omega_{k}^{(a)} E_{k}
$$


and the degree of $\omega_{k}^{(a)}$, as a polynomial in $X_{1}, \ldots, X_{k-1}$, is less than or equal to a. Moreover, the generating series $\widetilde{W}_{k}(y)=\sum_{a \geq 0} \omega_{k}^{(a)} y^{-a}$ satisfies

$$
\widetilde{W}_{k+1}(y)=-y+\frac{1}{2}+\frac{\left(y+X_{k}\right)^{2}-1}{\left(y-X_{k}\right)^{2}-1} \frac{\left(y-X_{k}\right)^{2}}{\left(y+X_{k}\right)^{2}}\left(\widetilde{W}_{k}(y)+y-\frac{1}{2}\right) .
$$

Proof. Observe that $\sum_{a \geq 0} E_{k} X_{k}^{a} E_{k} y^{-a}=E_{k} \frac{y}{y-X_{k}} E_{k}$, so to prove the Lemma it is enough to argue by induction on $k$ to show that $E_{k} \frac{1}{y-X_{k}} E_{k}=$ $\frac{1}{y} \widetilde{W}_{k}(y) E_{k}$, where $\widetilde{W}_{k}(y)$ and its coefficients are as above.

If $k=1$ then there is nothing to prove. Assume that $k>1$. Starting with the identity

$$
S_{k} \frac{1}{y-X_{k}}=\frac{1}{y-X_{k+1}} S_{k}+\frac{1}{y+X_{k}} E_{k} \frac{1}{y-X_{k}}-\frac{1}{\left(y-X_{k}\right)\left(y-X_{k+1}\right)} .
$$

Nazarov [Naz96, Prop. 4.2] proves that $E_{k+1} \frac{1}{y-X_{k+1}} E_{k+1}=\frac{1}{y} \widetilde{W}_{k+1}(y) E_{k+1}$, where $\widetilde{W}_{k+1}(y)$ satisfies the recurrence relation above. Nazarov assumes that he is working over the complex field (so, $R=\mathbb{C}$ ), however, his arguments are valid over an arbitrary ring. Nazarov also proves that if $R=\mathbb{C}$ then the coefficients of $\widetilde{W}_{k}(y)$ are central in $\mathscr{W}_{r, k-1}(\mathbf{u})$. We modify Nazarov's arguments to establish centrality for fields of positive characteristic.

By induction we may assume that the coefficients of $\widetilde{W}_{k}(y)$ commute with $E_{1}, \ldots, E_{k-2}$ and $S_{1}, \ldots, S_{k-2}$, so it is enough to show that the coefficients of $\widetilde{W}_{k+1}(y)$ commute with $E_{k-1}$ and $S_{k-1}$. Since $k \geq 2$ we can write

$$
\begin{aligned}
\frac{\widetilde{W}_{k+1}(y)+y-\frac{1}{2}}{\widetilde{W}_{k-1}(y)+y-\frac{1}{2}}=\frac{\mathcal{X}}{\mathcal{Y}}:= & \frac{\left(y+X_{k}\right)^{2}-1}{\left(y-X_{k}\right)^{2}-1} \frac{\left(y-X_{k}\right)^{2}}{\left(y+X_{k}\right)^{2}} \\
& \times \frac{\left(y+X_{k-1}\right)^{2}-1}{\left(y-X_{k-1}\right)^{2}-1} \frac{\left(y-X_{k-1}\right)^{2}}{\left(y+X_{k-1}\right)^{2}} .
\end{aligned}
$$

As $E_{k-1}$ and $S_{k-1}$ commute with $\mathscr{W}_{r, k-2}(\mathbf{u})$ it is enough to show that $E_{k-1} \frac{\mathcal{X}}{\mathcal{Y}}=\frac{\mathcal{X}}{\mathcal{Y}} E_{k-1}$ and $S_{k-1} \frac{\mathcal{X}}{\mathcal{Y}}=\frac{\mathcal{X}}{\mathcal{Y}} S_{k-1}$. Now, $E_{k-1} \frac{\mathcal{X}}{\mathcal{Y}}=\frac{\mathcal{X}}{\mathcal{Y}} E_{k-1}$ if and only if $\mathcal{Y} E_{k-1} \mathcal{X}=\mathcal{X} E_{k-1} \mathcal{Y}$, and this follows easily using relation 2.1(i).

To prove that $S_{k-1}$ commutes with $\frac{\mathcal{X}}{\mathcal{Y}}$ let

$$
\sum_{m \geq 0} a_{m} z^{m}=\frac{\left(1+X_{k-1} z\right)\left(1+X_{k} z\right)}{\left(1-X_{k-1} z\right)\left(1-X_{k} z\right)}
$$


where $z=-y^{-1}$ or $z=(y \pm 1)^{-1}$. Then $a_{0}=1, a_{1}=2\left(X_{k-1}+X_{k}\right)$, $a_{2}=2\left(X_{k-1}+X_{k}\right)^{2}$ and

$$
a_{m}=\left(X_{k-1}+X_{k}\right) a_{m-1}-X_{k-1} X_{k} a_{m-2}, \quad \text { for } m \geq 3 \text {. }
$$

Consequently, if $m \geq 1$ then $a_{m}=\left(X_{k-1}+X_{k}\right) f_{m}\left(X_{k-1}, X_{k}\right)$, for some $f_{m} \in R\left[X_{k-1}, X_{k}\right]$. Now, relation 2.1(e) implies that $S_{k-1}$ and $X_{k-1}+X_{k}$ commute. Therefore, by induction,

$$
\begin{aligned}
S_{k-1} a_{m}= & \left(X_{k-1}+X_{k}\right) S_{k-1} a_{m-1} \\
& \quad-\left(X_{k-1} X_{k} S_{k-1}+E_{k-1} X_{k}-X_{k} E_{k-1}\right) a_{m-2} \\
= & \left(X_{k-1}+X_{k}\right) a_{m-1} S_{k-1}-X_{k-1} X_{k} a_{m-2} S_{k-1} \\
= & a_{m} S_{k-1}
\end{aligned}
$$

as required.

Finally, it follows from the recurrence relation that $\omega_{k}^{(a)} \in R\left[X_{1}, \ldots\right.$, $\left.X_{k-1}\right]$ and that $\omega_{k}^{(a)}$ has total degree at most $a$ as a polynomial in $X_{1}, \ldots$, $X_{k-1}$.

Remark 4.16. To prove that the $\omega_{k}^{(a)} \in Z\left(\mathscr{W}_{r, k-1}(\mathbf{u})\right)$ Nazarov uses the identity

$$
\exp \left(\sum_{a \geq 0} 2\left(X_{k-1}^{2 a+1}+X_{k}^{2 a+1}\right) \frac{y^{-2 a-1}}{2 a+1}\right)=\frac{\left(y+X_{k-1}\right)\left(y+X_{k}\right)}{\left(y-X_{k-1}\right)\left(y-X_{k}\right)} .
$$

However, this formula is only valid in characteristic zero.

By Lemma 4.15, we have

$$
\widetilde{W}_{k}(y)+y-\frac{1}{2}=\left(\widetilde{W}_{1}(y)+y-\frac{1}{2}\right) \prod_{i=1}^{k-1} \frac{\left(y+X_{i}\right)^{2}-1}{\left(y-X_{i}\right)^{2}-1} \cdot \frac{\left(y-X_{i}\right)^{2}}{\left(y+X_{i}\right)^{2}}
$$

As the right hand side acts on each $v_{\mathfrak{t}}$ as multiplication by a scalar we can define $\widetilde{W}_{k}(y, \mathfrak{t}) \in R\left(\left(y^{-1}\right)\right)$ by $\widetilde{W}_{k}(y) v_{\mathfrak{t}}=\widetilde{W}_{k}(y, \mathfrak{t}) v_{\mathfrak{t}}$.

The next Proposition gives a representation theoretic interpretation of the rational functions $W_{k}(y, \mathfrak{t})$ which were introduced in Definition 4.5.

Proposition 4.17. Suppose that $\mathfrak{t} \in \mathscr{T}_{n}^{u d}(\lambda)$ and that $1 \leq k \leq n$. Then

$$
W_{k}(y, \mathfrak{t})=\widetilde{W}_{k}(y, \mathfrak{t}) .
$$


Proof. As $\Omega$ is u-admissible, by Lemma 3.8 we have

$$
\widetilde{W}_{1}(y, \mathfrak{t})+y-\frac{1}{2}=\left(y+\frac{1}{2}(-1)^{r+1}\right) \prod_{t=1}^{r} \frac{y+u_{t}}{y-u_{t}} .
$$

Consequently, we can rewrite the definition of $\widetilde{W}_{k}(y, \mathfrak{t})$ as

$$
\begin{aligned}
\widetilde{W}_{k}(y, \mathfrak{t})+y-\frac{1}{2}=(y & \left.-\frac{1}{2}(-1)^{r}\right) \cdot \prod_{t=1}^{r} \frac{\left(y+u_{t}\right)}{\left(y-u_{t}\right)} \\
& \times \prod_{i=1}^{k-1} \frac{\left(y+c_{\mathfrak{t}}(i)\right)^{2}-1}{\left(y-c_{\mathfrak{t}}(i)\right)^{2}-1} \cdot \frac{\left(y-c_{\mathfrak{t}}(i)\right)^{2}}{\left(y+c_{\mathfrak{t}}(i)\right)^{2}} .
\end{aligned}
$$

If $c_{\mathfrak{t}}(i)=-c_{\mathfrak{t}}(j)$, for some $1 \leq i, j \leq k-1$ with $i \neq j$, then

$$
\frac{\left(y+c_{\mathfrak{t}}(i)\right)^{2}-1}{\left(y-c_{\mathfrak{t}}(i)\right)^{2}-1} \cdot \frac{\left(y-c_{\mathfrak{t}}(i)\right)^{2}}{\left(y+c_{\mathfrak{t}}(i)\right)^{2}} \cdot \frac{\left(y+c_{\mathfrak{t}}(j)\right)^{2}-1}{\left(y-c_{\mathfrak{t}}(j)\right)^{2}-1} \cdot \frac{\left(y-c_{\mathfrak{t}}(j)\right)^{2}}{\left(y+c_{\mathfrak{t}}(j)\right)^{2}}=1 .
$$

Hence, in computing $\widetilde{W}_{k}(y, \mathfrak{t})$ we can assume that $\mathfrak{t}=\left(\mathfrak{t}_{1}, \ldots, \mathfrak{t}_{m}, \ldots\right.$, $\left.\mathfrak{t}_{k-1}, \ldots, \mathfrak{t}_{n}\right)$ where $m=\left|\mathfrak{t}_{k-1}\right|, \mathfrak{t}_{m}=\mathfrak{t}_{k-1}$ and $c_{\mathfrak{t}}(i)+c_{\mathfrak{t}}(i+1)=0$ for $m<i<k-1$ with $i-m$ odd (so $\mathfrak{t}_{i+1}$ is obtained by adding a box to $\mathfrak{t}_{i}$, for $1 \leq i<m$, and $\mathfrak{t}_{i}=\mathfrak{t}_{k-1}$ for $m \leq i \leq k-1$ with $i-m$ even). Let $\mathfrak{t}_{k-1}=\left(\mu^{(1)}, \mu^{(2)}, \ldots, \mu^{(r)}\right)$. Fix $t$ with $1 \leq t \leq r$ and, abusing notation, write $\beta \in \mu_{k}^{(t)}$ to indicate that $\beta=(k, j, t)$ is a node in row $k$ of $\mu^{(t)}$. Let $p_{1}=(k, 1, t), p_{2}=\left(k, \mu_{k}^{(t)}, t\right), p_{3}=\left(k, \mu_{k}^{(t)}+1, t\right)$ and $p_{4}=(k+1,1, t)$. Then

$$
\begin{aligned}
\prod_{\beta \in \mu_{k}^{(t)}} & \frac{\left(y+c^{\prime}(\beta)\right)^{2}-1}{\left(y-c^{\prime}(\beta)\right)^{2}-1} \cdot \frac{\left(y-c^{\prime}(\beta)\right)^{2}}{\left(y+c^{\prime}(\beta)\right)^{2}} \\
= & \prod_{\beta \in \mu_{k}^{(t)}} \frac{y-c^{\prime}(\beta)}{y-\left(c^{\prime}(\beta)+1\right)} \frac{y-c^{\prime}(\beta)}{y-\left(c^{\prime}(\beta)-1\right)} \frac{y+\left(c^{\prime}(\beta)+1\right)}{y+c^{\prime}(\beta)} \frac{y+\left(c^{\prime}(\beta)-1\right)}{y+c^{\prime}(\beta)} \\
= & \frac{y-c^{\prime}\left(p_{1}\right)}{y-c^{\prime}\left(p_{3}\right)} \frac{y-c^{\prime}\left(p_{2}\right)}{y-c^{\prime}\left(p_{4}\right)} \frac{y+c^{\prime}\left(p_{3}\right)}{y+c^{\prime}\left(p_{1}\right)} \frac{y+c^{\prime}\left(p_{4}\right)}{y+c^{\prime}\left(p_{2}\right)} \\
= & \frac{y-c^{\prime}\left(p_{1}\right)}{y+c^{\prime}\left(p_{1}\right)} \frac{y-c^{\prime}\left(p_{2}\right)}{y+c^{\prime}\left(p_{2}\right)} \frac{y+c^{\prime}\left(p_{3}\right)}{y-c^{\prime}\left(p_{3}\right)} \frac{y+c^{\prime}\left(p_{4}\right)}{y-c^{\prime}\left(p_{4}\right)}
\end{aligned}
$$

where for $\beta=(a, b, t)$ we write $c^{\prime}(\beta)=b-a+u_{t}$. Taking the product over all $k$ shows that

$$
\frac{\left(y+u_{t}\right)}{\left(y-u_{t}\right)} \prod_{\beta \in \mu^{(t)}} \frac{(y+c(\beta))^{2}-1}{(y-c(\beta))^{2}-1} \cdot \frac{(y-c(\beta))^{2}}{(y+c(\beta))^{2}}=\prod_{\alpha} \frac{y+c(\alpha)}{y-c(\alpha)},
$$


where, in the first product, every node is considered to be an addable node and, in the second product, $\alpha$ runs over the addable and removable nodes of $\mu^{(t)}$. Hence,

$$
\widetilde{W}_{k}(y, \mathfrak{t})+y-\frac{1}{2}=\left(y-\frac{1}{2}(-1)^{r}\right) \prod_{\alpha} \frac{y+c(\alpha)}{y-c(\alpha)},
$$

where $\alpha$ runs over the addable and removable nodes of $\mathfrak{t}_{k-1}=\left(\mu^{(1)}, \ldots\right.$, $\left.\mu^{(r)}\right)$.

Corollary 4.18. Suppose that $\mathfrak{t} \in \mathscr{T}_{n}^{u d}(\lambda)$ and that $1 \leq k<n$ and $a \geq 0$. Then $E_{k} X_{k}^{a} E_{k} v_{\mathfrak{t}}=\omega_{k}^{(a)} E_{k} v_{\mathfrak{t}}$.

Proof. If $\mathfrak{t}_{k-1} \neq \mathfrak{t}_{k+1}$ then $E_{k} X_{k}^{i} E_{k} v_{\mathfrak{t}}=0=\omega_{k}^{(i)} E_{k} v_{\mathfrak{t}}$, so we may assume that $\mathfrak{t}_{k-1}=\mathfrak{t}_{k+1}$. Now, by definition, $e_{\mathfrak{t u}}(k)=\sqrt{e_{\mathfrak{t} \mathfrak{t}}(k)} \sqrt{e_{\mathfrak{u} \mathfrak{u}}(k)}$. So

$$
\begin{aligned}
E_{k} \frac{y}{y-X_{k}} E_{k} v_{\mathfrak{t}} & =E_{k} \sum_{\mathfrak{u}^{k} \mathfrak{t}} \frac{y}{y-c_{\mathfrak{u}}(k)} e_{\mathfrak{t} \mathfrak{u}}(k) v_{\mathfrak{u}} \\
& =\sum_{\substack{\mathfrak{w} \sim \mathfrak{u} \\
\sim}} \sum_{\mathfrak{u} \sim \mathfrak{i}} \frac{y}{y-c_{\mathfrak{u}}(k)} e_{\mathfrak{u} \mathfrak{w}}(k) e_{\mathfrak{t} \mathfrak{u}}(k) v_{\mathfrak{w}} \\
& =\sum_{\substack{\mathfrak{w} \sim \mathfrak{t} \\
\sim}}\left(\sum_{\mathfrak{u} \sim \mathfrak{t}} \frac{y}{y-c_{\mathfrak{u}}(k)} e_{\mathfrak{u} \mathfrak{u}}(k)\right) e_{\mathfrak{t w}}(k) v_{\mathfrak{w}} \\
& =W_{k}(y, \mathfrak{t}) E_{k} v_{\mathfrak{t}}=\widetilde{W}_{k}(y, \mathfrak{t}) E_{k} v_{\mathfrak{t}},
\end{aligned}
$$

by Proposition 4.17. By Lemma 4.15, $\omega_{k}^{(a)} \in R\left[X_{1}, \ldots, X_{k-1}\right]$, so $\omega_{k}^{(a)} v_{\mathfrak{t}}=$ $\omega_{k}^{(a)} v_{\mathfrak{u}}$ whenever $\mathfrak{t} \stackrel{k}{\sim} \mathfrak{u}$. Therefore,

$$
\begin{aligned}
E_{k} \frac{y}{y-X_{k}} E_{k} v_{\mathfrak{t}} & =\sum_{\substack{\mathfrak{u} \sim \mathfrak{t} \\
\sim}} e_{\mathfrak{t} \mathfrak{u}}(k) \widetilde{W}_{k}(y, \mathfrak{t}) v_{\mathfrak{u}}=\sum_{\substack{\mathfrak{u} \\
\mathfrak{u} \mathfrak{t}}} e_{\mathfrak{t u}}(k) \widetilde{W}_{k}(y, \mathfrak{u}) v_{\mathfrak{u}} \\
& =\widetilde{W}_{k}(y) \sum_{\substack{\mathfrak{u} \sim \mathfrak{t} \\
\mathfrak{w}}} e_{\mathfrak{t u}}(k) v_{\mathfrak{u}}=\widetilde{W}_{k}(y) E_{k} v_{\mathfrak{t}} .
\end{aligned}
$$

Comparing the coefficient of $y^{-a}$, for $a \geq 0$, on both sides of the last equation proves the Corollary.

LEMmA 4.19. Suppose that $\mathfrak{t} \in \mathscr{T}_{n}^{u d}(\lambda)$ with $\mathfrak{t}_{k-1}=\mathfrak{t}_{k+1}$ and $\mathfrak{t}_{k}=\mathfrak{t}_{k+2}$. Then $e_{\mathfrak{t} \mathfrak{t}}(k) e_{\mathfrak{t t}}(k+1)=1$. 
Proof. The recursion formula of Lemma 4.15 and Proposition 4.17 show that

$$
W_{k+1}(y, \mathfrak{t})+y-\frac{1}{2}=\left(W_{k}(y, \mathfrak{t})+y-\frac{1}{2}\right) \frac{\left(y-c_{\mathfrak{t}}(k)\right)^{2}}{\left(y+c_{\mathfrak{t}}(k)\right)^{2}} \frac{\left(y+c_{\mathfrak{t}}(k)\right)^{2}-1}{\left(y-c_{\mathfrak{t}}(k)\right)^{2}-1},
$$

and, by definition,

$$
W_{k}(y, \mathfrak{t})+y-\frac{1}{2}=\left(y-\frac{1}{2}(-1)^{r}\right) \prod_{\substack{k \\ \mathfrak{u} \sim \mathfrak{t}}} \frac{y+c_{\mathfrak{u}}(k)}{y-c_{\mathfrak{u}}(k)} .
$$

Thus,

$$
\begin{aligned}
\frac{W_{k+1}(y, \mathfrak{t})+y-\frac{1}{2}}{y}=(1 & \left.-\frac{1}{2 y}(-1)^{r}\right) \frac{y-c_{\mathfrak{t}}(k)}{y+c_{\mathfrak{t}}(k)} \frac{\left(y+c_{\mathfrak{t}}(k)\right)^{2}-1}{\left(y-c_{\mathfrak{t}}(k)\right)^{2}-1} \\
& \times \prod_{\substack{\mathfrak{k} \mathfrak{k} \mathfrak{k}, \mathfrak{u} \neq \mathfrak{t}\\
}} \frac{y+c_{\mathfrak{u}}(k)}{y-c_{\mathfrak{u}}(k)} .
\end{aligned}
$$

Taking residues at $y=-c_{\mathfrak{t}}(k)=c_{\mathfrak{t}}(k+1)$ on both sides of this equation, we have

$$
\begin{aligned}
& e_{\mathfrak{t t}}(k+1)=\frac{2 c_{\mathfrak{t}}(k)+(-1)^{r}}{4 c_{\mathfrak{t}}(k)^{2}-1} \prod_{\substack{k \\
\mathfrak{u} \sim \mathfrak{t}, \mathfrak{u} \neq \mathfrak{t}}} \frac{c_{\mathfrak{t}}(k)-c_{\mathfrak{u}}(k)}{c_{\mathfrak{t}}(k)+c_{\mathfrak{u}}(k)} \\
& =\frac{1}{2 c_{\mathfrak{t}}(k)-(-1)^{r}} \prod_{\substack{\mathfrak{u} \sim \mathfrak{t}, \mathfrak{u} \neq \mathfrak{t} \\
c_{\mathfrak{t}}(k)+c_{\mathfrak{u}}(k)}} \frac{c_{\mathfrak{t}}(k)-c_{\mathfrak{u}}(k)}{c_{\mathfrak{t}}(k)} .
\end{aligned}
$$

where the last equality uses (4.8).

We remark that the condition $\mathfrak{t}_{k}=\mathfrak{t}_{k+2}$ is needed in Lemma 4.19 only because $e_{\mathfrak{t t}}(k+1)$ is not defined without this assumption.

Lemma 4.20. Fix an integer $k$ with $1 \leq k<n-1$ and suppose that $\mathfrak{t}, \mathfrak{u}, \mathfrak{w} \in \mathscr{T}_{n}^{u d}(\lambda)$ are updown $\lambda$-tableaux such that $\mathfrak{t}_{k-1}=\mathfrak{t}_{k+1}, \mathfrak{t}_{k}=\mathfrak{t}_{k+2}$, $\mathfrak{u} \stackrel{k+1}{\sim} \mathfrak{t}, \mathfrak{w} \stackrel{k}{\sim} \mathfrak{t}$ and that $S_{k} \mathfrak{u}$ and $S_{k+1} \mathfrak{w}$ are both defined with $S_{k} \mathfrak{u}=S_{k+1} \mathfrak{w}$. Then $b_{\mathfrak{u}}(k)^{2} e_{\mathfrak{u} u}(k+1)=b_{\mathfrak{w}}(k+1)^{2} e_{\mathfrak{w} w}(k)$.

Proof. Let $\sigma=\mathfrak{t}_{k} \ominus \mathfrak{t}_{k-1}$ and $\tau=\mathfrak{u}_{k+1} \ominus \mathfrak{t}_{k} . \quad S_{k} \mathfrak{u}=S_{k+1} \mathfrak{w}$ implies $\tau=\mathfrak{w}_{k} \ominus \mathfrak{t}_{k-1}$. Then, by (4.8),

$$
e_{\mathfrak{w} \mathfrak{w}}(k)=\left(2 c(\tau)-(-1)^{r}\right) \prod_{\alpha} \frac{c(\tau)+c(\alpha)}{c(\tau)-c(\alpha)},
$$


where $\alpha$ runs over the addable and removable nodes of $\mathfrak{t}_{k-1}=\mathfrak{w}_{k-1}$ with $c(\alpha) \neq c(\tau)$ and, similarly,

$$
e_{\mathfrak{u}}(k+1)=\left(2 c(\tau)-(-1)^{r}\right) \prod_{\alpha} \frac{c(\tau)+c(\alpha)}{c(\tau)-c(\alpha)}
$$

where $\alpha$ runs over all addable and removable nodes of $\mathfrak{t}_{k}=\mathfrak{u}_{k}$ with $c(\alpha) \neq$ $c(\tau)$. We have $e_{\mathfrak{w} \mathfrak{w}}(k)=\operatorname{Res}_{y=c(\tau)} \frac{W_{k}(y, \mathfrak{t})+y-1 / 2}{y}$ and $e_{\mathfrak{u u}}(k+1)=$ $\operatorname{Res}_{y=c(\tau)} \frac{W_{k+1}(y, \mathfrak{t})+y-1 / 2}{y}$. Further, by Lemma 4.15 and Proposition 4.17, we have

$$
W_{k+1}(y, \mathfrak{t})+y-\frac{1}{2}=\left(W_{k}(y, \mathfrak{t})+y-\frac{1}{2}\right) \frac{(y+c(\sigma))^{2}-1}{(y-c(\sigma))^{2}-1} \frac{(y-c(\sigma))^{2}}{(y+c(\sigma))^{2}} .
$$

It follows that

$$
\frac{e_{\mathfrak{u u}}(k+1)}{e_{\mathfrak{w} \mathfrak{w}}(k)}=\frac{(c(\sigma)+c(\tau))^{2}-1}{(c(\sigma)+c(\tau))^{2}} \frac{(c(\tau)-c(\sigma))^{2}}{(c(\tau)-c(\sigma))^{2}-1}=\frac{b_{\mathfrak{w}}(k+1)^{2}}{b_{\mathfrak{u}}(k)^{2}},
$$

where the last equality follows from the definitions because $\left(c_{\mathfrak{u}}(k), c_{\mathfrak{u}}(k+\right.$ $\left.1), c_{\mathfrak{u}}(k+2)\right)=(c(\sigma), c(\tau),-c(\tau))$ and $\left(c_{\mathfrak{w}}(k), c_{\mathfrak{w}}(k+1), c_{\mathfrak{w}}(k+2)\right)=$ $(c(\tau),-c(\tau), c(\sigma))$.

The following combinatorial identities will be used in the proof of Theorem 4.13 .

Proposition 4.21. Suppose that $\mathfrak{t}, \mathfrak{u}^{\prime} \in \mathscr{T}_{n}^{u d}(\lambda)$ with $\mathfrak{t}_{k-1}=\mathfrak{t}_{k+1}, \mathfrak{t}_{k} \neq$ $\mathfrak{t}_{k+2}, \mathfrak{u}^{\prime} \stackrel{k}{\sim} \mathfrak{t}$ and $\mathfrak{u}^{\prime} \neq \mathfrak{t}$, where $1 \leq k<n-1$. Let $\tilde{\mathfrak{t}} \in \mathscr{T}_{n}^{u d}(\lambda)$ be the updown tableau which is uniquely determined by the conditions $\tilde{\mathfrak{t}} \stackrel{k}{\sim} \mathfrak{t}$ and $\tilde{\mathfrak{t}}_{k}=\mathfrak{t}_{k+2}$. Then the following identities hold:

a) $\sum_{\substack{k \\ \mathfrak{u} \sim \mathfrak{t}}} \frac{e_{\mathfrak{u} \mathfrak{u}}(k)}{c_{\mathfrak{t}}(k)+c_{\mathfrak{u}}(k)}=1+\frac{1}{2 c_{\mathfrak{t}}(k)}$

b) $\sum_{\substack{\mathfrak{u} \sim \mathfrak{t}\\}} \frac{e_{\mathfrak{u u}}(k)}{\left(c_{\mathfrak{t}}(k)+c_{\mathfrak{u}}(k)\right)^{2}}=\left(1-\frac{1}{4 c_{\mathfrak{t}}(k)^{2}}\right) \frac{1}{e_{\mathfrak{t} \mathfrak{t}}(k)}+\frac{1}{2 c_{\mathfrak{t}}(k)^{2}}$,

c) $\sum_{\substack{\mathfrak{u} \sim \mathfrak{t} \\ \sim}} \frac{e_{\mathfrak{u} \mathfrak{u}}(k)}{\left(c_{\mathfrak{t}}(k)+c_{\mathfrak{u}}(k)\right)\left(c_{\mathfrak{u}}(k)+c_{\mathfrak{u}^{\prime}}(k)\right)}=\frac{1}{2 c_{\mathfrak{t}}(k) c_{\mathfrak{u}^{\prime}}(k)}$. 
Proof. It follows from (4.9) and Definition 4.7 that

$$
\frac{W_{k}(y, \mathfrak{t})}{y}=\sum_{\substack{k \\ \mathfrak{u} \mathfrak{t}}} \frac{e_{\mathfrak{u}}(k)}{y-c_{\mathfrak{u}}(k)}
$$

Evaluating both sides at $y=-c_{\mathfrak{t}}(k)$ and using (4.5) gives (a).

By Proposition 4.17 and Corollary 4.18 we have

$$
E_{k} \frac{1}{\left(y-X_{k}\right)\left(v-X_{k}\right)} E_{k} v_{\mathfrak{t}}=\frac{1}{v-y}\left(\frac{W_{k}(y, \mathfrak{t})}{y}-\frac{W_{k}(v, \mathfrak{t})}{v}\right) E_{k} v_{\mathfrak{t}}
$$

Comparing the coefficients of $v_{\mathfrak{t}}$ on both sides of this equation we obtain

$$
\sum_{\substack{\mathfrak{u} \sim \mathfrak{t} \\ \mathfrak{u}}} \frac{e_{\mathfrak{u} u}(k)}{\left(y-c_{\mathfrak{u}}(k)\right)\left(v-c_{\mathfrak{u}}(k)\right)}=\frac{1}{v-y}\left\{\frac{W_{k}(y, \mathfrak{t})}{y}-\frac{W_{k}(v, \mathfrak{t})}{v}\right\} .
$$

Setting $y=-c_{\mathfrak{t}}(k)$ we obtain

$$
\begin{aligned}
& \sum_{\substack{\mathfrak{u} \sim \mathfrak{t}\\
}} \frac{e_{\mathfrak{u} u}(k)}{\left(c_{\mathfrak{t}}(k)+c_{\mathfrak{u}}(k)\right)\left(v-c_{\mathfrak{u}}(k)\right)} \\
& \quad=\frac{1}{v+c_{\mathfrak{t}}(k)}\left\{\left(\frac{W_{k}(v, \mathfrak{t})}{v}+1-\frac{1}{2 v}\right)+\left(\frac{1}{2 c_{\mathfrak{t}}(k)}+\frac{1}{2 v}\right)\right\} \\
& \quad=\frac{2 v-(-1)^{r}}{2 v\left(v+c_{\mathfrak{t}}(k)\right)} \prod_{\substack{k \\
\mathfrak{u} \sim \mathfrak{t}}} \frac{v+c_{\mathfrak{u}}(k)}{v-c_{\mathfrak{u}}(k)}+\frac{1}{2 c_{\mathfrak{t}}(k) v} .
\end{aligned}
$$

Setting $v=-c_{\mathfrak{u}^{\prime}}(k)$ gives (c). Now we set $v=-c_{\mathfrak{t}}(k)$. Then it gives

$$
\sum_{\substack{\mathfrak{u} \sim \mathfrak{t} \\ \mathfrak{u} \sim \mathfrak{t}}} \frac{e_{\mathfrak{u u}}(k)}{\left(c_{\mathfrak{t}}(k)+c_{\mathfrak{u}}(k)\right)^{2}}=\frac{2 c_{\mathfrak{t}}(k)+(-1)^{r}}{4 c_{\mathfrak{t}}(k)^{2}} \prod_{\substack{\mathfrak{k} \\ \mathfrak{u} \sim \mathfrak{t} \\ \mathfrak{u} \neq \mathfrak{t}}} \frac{c_{\mathfrak{t}}(k)-c_{\mathfrak{u}}(k)}{c_{\mathfrak{t}}(k)+c_{\mathfrak{u}}(k)}+\frac{1}{2 c_{\mathfrak{t}}(k)^{2}} .
$$

On the other hand, multiplying the reciprocal of $(4.8)$ by $\left(1-\frac{1}{4 c_{\mathfrak{t}}(k)^{2}}\right)$ gives

$$
\left(1-\frac{1}{4 c_{\mathfrak{t}}(k)^{2}}\right) \frac{1}{e_{\mathfrak{t} \mathfrak{t}}(k)}=\frac{2 c_{\mathfrak{t}}(k)+(-1)^{r}}{4 c_{\mathfrak{t}}(k)^{2}} \prod_{\substack{k \\ \mathfrak{u} \mathfrak{t}, \mathfrak{u} \neq \mathfrak{t}}} \frac{c_{\mathfrak{t}}(k)-c_{\mathfrak{u}}(k)}{c_{\mathfrak{t}}(k)+c_{\mathfrak{u}}(k)} .
$$

Combining these two equations gives (b). 
We are now ready to start checking that the action of $\mathscr{W}_{r, n}(\mathbf{u})$ on $\Delta(\lambda)$ respects the relations of $\mathscr{W}_{r, n}(\mathbf{u})$. We break the proof into several lemmas and propositions.

LemmA 4.22. Suppose $\mathfrak{t} \in \mathscr{T}_{n}^{u d}(\lambda)$. Then

a) $E_{i}^{2} v_{\mathfrak{t}}=\omega_{0} E_{i} v_{\mathfrak{t}}$, for $1 \leq i<n$.

b) $E_{1} X_{1}^{a} E_{1} v_{\mathfrak{t}}=\omega_{a} E_{1} v_{\mathfrak{t}}$, for $a>0$.

c) $\left(X_{1}-u_{1}\right)\left(X_{1}-u_{2}\right) \cdots\left(X_{1}-u_{r}\right) v_{\mathfrak{t}}=0$.

d) $X_{i} X_{j} v_{\mathfrak{t}}=X_{j} X_{i} v_{\mathfrak{t}}$ for $1 \leq i, j \leq n$.

e) $E_{i}\left(X_{i}+X_{i+1}\right) v_{\mathfrak{t}}=\left(X_{i}+X_{i+1}\right) E_{i} v_{\mathfrak{t}}=0,1 \leq i \leq n-1$.

f) $\left(S_{i} X_{i}-X_{i+1} S_{i}\right) v_{\mathfrak{t}}=\left(E_{i}-1\right) v_{\mathfrak{t}}=\left(X_{i} S_{i}-S_{i} X_{i+1}\right) v_{\mathfrak{t}}$, for $1 \leq i \leq n-1$.

g) $E_{k} E_{l} v_{\mathfrak{t}}=E_{l} E_{k} v_{\mathfrak{t}}$ if $|k-l|>1$.

h) $E_{k} X_{l} v_{\mathfrak{t}}=X_{l} E_{k} v_{\mathfrak{t}}$ if $l \neq k, k+1$.

i) $S_{k} X_{l} v_{\mathfrak{t}}=X_{l} S_{k} v_{\mathfrak{t}}$ if $l \neq k, k+1$.

Proof. As $\omega_{0}=\omega_{1}^{(0)}$ and $\omega_{a}=\omega_{1}^{(a)}$ by Lemma 4.15, parts (a) and (b) have already been proved in Corollary 4.18. Parts (c)-(f) follow directly from the definitions of the actions. If $|k-l|>1$ then (4.12)(e) shows that (g) holds. Assume now that $l \neq k, k+1$. If $\mathfrak{t}_{k-1} \neq \mathfrak{t}_{k+1}$ then $c_{S_{k}} \mathfrak{t}(l)=c_{\mathfrak{t}}(l)$. If $\mathfrak{u} \stackrel{k}{\sim} \mathfrak{t}$ then $c_{\mathfrak{u}}(l)=c_{\mathfrak{t}}(l)$. Combining the last two statements forces $(\mathrm{h})$ and (i) to be true.

Lemma 4.23. Suppose $\mathfrak{t} \in \mathscr{T}_{n}^{u d}(\lambda)$. Then $E_{k} E_{k \pm 1} E_{k} v_{\mathfrak{t}}=E_{k} v_{\mathfrak{t}}$.

Proof. We only prove that $E_{k} E_{k+1} E_{k} v_{\mathfrak{t}}=E_{k} v_{\mathfrak{t}}$, since the argument for the case $E_{k} E_{k-1} E_{k} v_{\mathfrak{t}}=E_{k} v_{\mathfrak{t}}$ is almost identical.

We may assume $\mathfrak{t}_{k-1}=\mathfrak{t}_{k+1}$ since, otherwise, $E_{k} E_{k+1} E_{k} v_{\mathfrak{t}}=0=E_{k} v_{\mathfrak{t}}$. Let $\tilde{\mathfrak{t}}$ be the unique $n$-updown tableau such that $\tilde{\mathfrak{t}} \stackrel{k}{\sim} \mathfrak{t}$ and $\tilde{\mathfrak{t}}_{k}=\mathfrak{t}_{k+2}$. We have

$$
E_{k} E_{k+1} E_{k} v_{\mathfrak{t}}=e_{\mathfrak{\mathfrak { t }}}(k) e_{\tilde{\mathfrak{t}} \mathfrak{t}}(k+1) \sum_{\substack{k, \tilde{\mathfrak{u}} \\ \sim}} e_{\tilde{\mathfrak{t}} \mathfrak{u}}(k) v_{\mathfrak{u}}=e_{\tilde{\mathfrak{t}} \mathfrak{t}}(k) e_{\tilde{\mathfrak{t}} \mathfrak{t}}(k+1) \sum_{\substack{\mathfrak{u} \sim \mathfrak{t}}} e_{\mathfrak{t} \mathfrak{u}}(k) v_{\mathfrak{u}} .
$$

Hence, $E_{k} E_{k+1} E_{k} v_{\mathfrak{t}}=E_{k} v_{\mathfrak{t}}$ by Lemma 4.19 .

It remains to check relations (a), (b)(i), (b)(ii), (d)(i) and (g) from Definition 2.1. 
Lemma 4.24. Suppose that $\mathfrak{t} \in \mathscr{T}_{n}^{u d}(\lambda)$. Then $S_{k}^{2} v_{\mathfrak{t}}=v_{\mathfrak{t}}$.

Proof. Case 1. $\mathfrak{t}_{k-1} \neq \mathfrak{t}_{k+1}$ : If $S_{k} \mathfrak{t}$ is not defined then $a_{\mathfrak{t}}(k) \in\{-1,1\}$ and $b_{\mathfrak{t}}(k)=0$, which implies $S_{k}^{2} v_{\mathfrak{t}}=v_{\mathfrak{t}}$. If $S_{k} \mathfrak{t} \in \mathscr{T}_{n}^{u d}(\lambda)$ then by the choice of the square roots in $(4.12)(\mathrm{a})$ we have

$$
S_{k}^{2} v_{\mathfrak{t}}=\left(a_{\mathfrak{t}}(k)^{2}+b_{\mathfrak{t}}(k) b_{S_{k} \mathfrak{t}}(k)\right) v_{\mathfrak{t}}+\left(a_{\mathfrak{t}}(k)+a_{S_{k} \mathfrak{t}}(k)\right) b_{\mathfrak{t}}(k) v_{S_{k} \mathfrak{t}}=v_{\mathfrak{t}} .
$$

Case 2. $\mathfrak{t}_{k-1}=\mathfrak{t}_{k+1}$ : We have $S_{k}^{2} v_{\mathfrak{t}}=\sum_{\mathfrak{u} \sim \mathfrak{t}}\left(\sum_{\mathfrak{v} \sim \mathfrak{t}} s_{\mathfrak{t v}}(k) s_{\mathfrak{v} \mathfrak{u}}(k)\right) v_{\mathfrak{u}}$. So, the coefficient of $v_{\mathfrak{t}}$ in $S_{k}^{2} \mathfrak{v}_{s}$ is

$$
\sum_{\substack{\mathfrak{u} \sim \mathfrak{t}}} s_{\mathfrak{t} \mathfrak{u}}(k) s_{\mathfrak{u t}}(k)=\sum_{\substack{\mathfrak{u} \sim \mathfrak{t} \\\left(c_{\mathfrak{t}}(k)+c_{\mathfrak{u}}(k)\right)^{2}}} \frac{e_{\mathfrak{t t}}(k) e_{\mathfrak{u} \mathfrak{u}}(k)}{2 c_{\mathfrak{t}}(k)^{2}}+\frac{1}{4 c_{\mathfrak{t}}(k)^{2}}=1
$$

where the last equality follows by rearranging Proposition 4.21 (b). If $\mathfrak{u} \stackrel{k}{\sim} \mathfrak{t}$ and $\mathfrak{u} \neq \mathfrak{t}$ then the coefficient of $v_{\mathfrak{u}}$ in $S_{k}^{2} v_{\mathfrak{t}}$ is

$$
\begin{aligned}
\sum_{\substack{k \\
\mathfrak{v} \sim \mathfrak{t}}} s_{\mathfrak{t} \mathfrak{v}}(k) s_{\mathfrak{v} \mathfrak{u}}(k)= & \sum_{\substack{\mathfrak{v} \sim \mathfrak{t} \\
\mathfrak{t} \neq \mathfrak{v} \neq \mathfrak{u}}} \frac{e_{\mathfrak{t} \mathfrak{v}}(k) e_{\mathfrak{v} \mathfrak{u}}(k)}{\left(c_{\mathfrak{t}}(k)+c_{\mathfrak{v}}(k)\right)\left(c_{\mathfrak{v}}(k)+c_{\mathfrak{u}}(k)\right)} \\
& \quad+\frac{\left(e_{\mathfrak{t} \mathfrak{t}}(k)-1\right) e_{\mathfrak{t} \mathfrak{u}}(k)}{2 c_{\mathfrak{t}}(k)\left(c_{\mathfrak{t}}(k)+c_{\mathfrak{u}}(k)\right)}+\frac{\left(e_{\mathfrak{u} \mathfrak{u}}(k)-1\right) e_{\mathfrak{t u}}(k)}{2 c_{\mathfrak{u}}(k)\left(c_{\mathfrak{t}}(k)+c_{\mathfrak{u}}(k)\right)} \\
= & e_{\mathfrak{t} \mathfrak{u}}(k)\left(\sum_{\mathfrak{v} \stackrel{k}{\sim}} \frac{e_{\mathfrak{v} \mathfrak{v}}(k)}{\left(c_{\mathfrak{t}}(k)+c_{\mathfrak{v}}(k)\right)\left(c_{\mathfrak{v}}(k)+c_{\mathfrak{u}}(k)\right)}-\frac{1}{2 c_{\mathfrak{t}}(k) c_{\mathfrak{u}}(k)}\right) \\
= & 0
\end{aligned}
$$

by Proposition $4.21(\mathrm{c})$. Therefore, $S_{k}^{2} v_{\mathfrak{t}}=v_{\mathfrak{t}}$.

The next two Propositions prove that the action of $\mathscr{W}_{r, n}(\mathbf{u})$ on $\Delta(\lambda)$ respects the tangle relations $2.1(\mathrm{~g})$.

Proposition 4.25. For any $\mathfrak{t} \in \mathscr{T}_{n}^{u d}(\lambda), E_{k} S_{k} v_{\mathfrak{t}}=E_{k} v_{\mathfrak{t}}=S_{k} E_{k} v_{\mathfrak{t}}$.

Proof. Suppose that $\mathfrak{t}_{k-1} \neq \mathfrak{t}_{k+1}$. Then either $S_{k} \mathfrak{t}$ is not defined, or $\left(S_{k} \mathfrak{t}\right)_{k-1} \neq\left(S_{k} \mathfrak{t}\right)_{k+1}$. In either case, we have $E_{k} S_{k} v_{\mathfrak{t}}=E_{k} v_{\mathfrak{t}}=S_{k} E_{k} v_{\mathfrak{t}}=0$. Suppose $\mathfrak{t}_{k-1}=\mathfrak{t}_{k+1}$. Then

$$
S_{k} E_{k} v_{\mathfrak{t}}=\sum_{\substack{k \\ \mathfrak{u} \sim \mathfrak{t}}} e_{\mathfrak{t u}}(k) S_{k} v_{\mathfrak{u}}=\sum_{\substack{\mathfrak{u}^{\prime} k \mathfrak{u} \\ \sim \mathfrak{u} \sim \mathfrak{t}}} \sum_{\substack{\mathfrak{u} \mathfrak{u}^{\prime}\\}}(k) e_{\mathfrak{t u}}(k) v_{\mathfrak{u}^{\prime}}
$$


By Proposition 4.21(a), we have

$$
\begin{aligned}
& \sum_{\substack{k \\
\mathfrak{u} \sim \mathfrak{t}}} e_{\mathfrak{t u}}(k) s_{\mathfrak{u} \mathfrak{u}^{\prime}}(k)=\sum_{\substack{k \\
\mathfrak{u} \sim \mathfrak{t}, \mathfrak{u} \neq \mathfrak{u}^{\prime}}} \frac{e_{\mathfrak{t u}}(k) e_{\mathfrak{u} \mathfrak{u}^{\prime}}(k)}{c_{\mathfrak{u}}(k)+c_{\mathfrak{u}^{\prime}}(k)}+e_{\mathfrak{t} \mathfrak{u}^{\prime}}(k) \frac{e_{\mathfrak{u}^{\prime} \mathfrak{u}^{\prime}}(k)-1}{2 c_{\mathfrak{u}^{\prime}}(k)}
\end{aligned}
$$

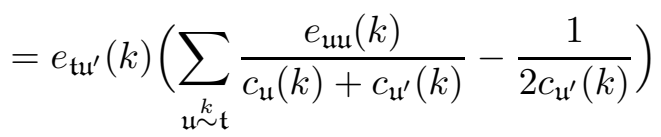

$$
\begin{aligned}
& =e_{\mathfrak{t} \mathfrak{u}^{\prime}}(k) \text {. }
\end{aligned}
$$

Hence, $S_{k} E_{k} v_{\mathfrak{t}}=E_{k} v_{\mathfrak{t}}$. One can prove that $E_{k} S_{k} v_{\mathfrak{t}}=E_{k} v_{\mathfrak{t}}$ similarly.

Proposition 4.26. Suppose that $\mathfrak{t} \in \mathscr{T}_{n}^{u d}(\lambda)$. Then

a) $S_{k} E_{k+1} E_{k} v_{\mathfrak{t}}=S_{k+1} E_{k} v_{\mathfrak{t}}$.

b) $E_{k+1} E_{k} S_{k+1} v_{\mathfrak{t}}=E_{k+1} S_{k} v_{\mathfrak{t}}$.

Proof. (a) We may assume that $\mathfrak{t}_{k-1}=\mathfrak{t}_{k+1}$ since otherwise $S_{k} E_{k+1}$ $E_{k} v_{\mathfrak{t}}=S_{k+1} E_{k} v_{\mathfrak{t}}=0$. Let $\tilde{\mathfrak{t}} \in \mathscr{T}_{n}^{u d}(\lambda)$ be the unique updown tableau such that $\tilde{\mathfrak{t}} \stackrel{k}{\sim} \mathfrak{t}$ and $\tilde{\mathfrak{t}}_{k}=\mathfrak{t}_{k+2}$. We have

$$
\begin{aligned}
& S_{k} E_{k+1} E_{k} v_{\mathfrak{t}}=e_{\mathfrak{t} \mathfrak{t}}(k) e_{\tilde{\mathfrak{t}} \mathfrak{t}}(k+1)\left(s_{\tilde{\mathfrak{t}} \mathfrak{t}}(k) v_{\tilde{\mathfrak{t}}}+\sum_{\substack{k \\
\mathfrak{u} \tilde{\mathfrak{t}}, \mathfrak{u} \neq \tilde{\mathfrak{t}}}} s_{\tilde{\mathfrak{t}} \mathfrak{u}}(k) v_{\mathfrak{u}}\right) \\
& +\sum_{\substack{k+1 \tilde{\mathfrak{t}}, \mathfrak{u} \neq \tilde{\mathfrak{t}}\\
}} e_{\mathfrak{\mathfrak { t }}}(k) e_{\tilde{\mathfrak{t}}_{\mathfrak{u}}}(k+1)\left(a_{\mathfrak{u}}(k) v_{\mathfrak{u}}+b_{\mathfrak{u}}(k) v_{S_{k} \mathfrak{u}}\right) .
\end{aligned}
$$

Observe that if $S_{k} \mathfrak{u}$ is defined, for $\mathfrak{u}$ in the second sum, then $\left(S_{k} \mathfrak{u}^{\prime}\right)_{k} \neq \mathfrak{t}_{k+2}$ and $\mathfrak{w}=S_{k+1} S_{k} \mathfrak{u}$ is also defined. Further, we have $\mathfrak{w} \stackrel{k}{\sim} \tilde{\mathfrak{t}}$ and $\mathfrak{w} \neq \mathfrak{t}$. Similarly,

$$
\begin{aligned}
S_{k+1} E_{k} v_{\mathfrak{t}}=e_{\mathfrak{t} \mathfrak{t}} & (k)\left(s_{\tilde{\mathfrak{t}} \mathfrak{t}}(k+1) v_{\tilde{\mathfrak{t}}}+\sum_{\substack{k+1 \tilde{\mathfrak{u}}, \mathfrak{u} \neq \tilde{\mathfrak{t}} \\
s_{\tilde{\mathfrak{t}} \mathfrak{u}}}}(k+1) v_{\mathfrak{u}}\right) \\
& +\sum_{\substack{\mathfrak{k} \sim \tilde{\mathfrak{t}}, \mathfrak{u} \neq \tilde{\mathfrak{t}}\\
}} e_{\mathfrak{t} \mathfrak{u}}(k)\left(a_{\mathfrak{u}}(k+1) v_{\mathfrak{u}}+b_{\mathfrak{u}}(k+1) v_{S_{k+1} \mathfrak{u}}\right) .
\end{aligned}
$$

We now compare the coefficients of $v_{\mathfrak{u}}$ in $S_{k} E_{k+1} E_{k} v_{\mathfrak{t}}$ and in $S_{k+1} E_{k} v_{\mathfrak{t}}$. First, observe that $e_{\tilde{\mathfrak{t}}}(k) e_{\tilde{\mathfrak{t}} \mathfrak{t}}(k+1)=1$ by Lemma 4.19 . 
Case 1. $\mathfrak{u}=\tilde{\mathfrak{t}}$ : Since $c_{\mathfrak{t}}(k)=-c_{\mathfrak{t}}(k+1)$, the definitions and the remarks above show that the coefficient of $v_{\mathfrak{u}}$ in $S_{k} E_{k+1} E_{k} v_{\mathfrak{t}}$ is equal to

$$
e_{\mathfrak{t} \mathfrak{t}}(k) e_{\tilde{\mathfrak{t} \mathfrak{t}}}(k+1) s_{\tilde{\mathfrak{t} \mathfrak{t}}}(k)=e_{\mathfrak{t} \mathfrak{t}}(k) \frac{1-e_{\tilde{\mathfrak{t}}}(k+1)}{2 c_{\tilde{\mathfrak{t}}}(k)}=e_{\tilde{\mathfrak{t}}}(k) s_{\tilde{\mathfrak{t}} \mathfrak{t}}(k+1),
$$

which is the coefficient of $v_{\mathfrak{u}}$ in $S_{k+1} E_{k} v_{\mathfrak{t}}$.

Case 2. $\mathfrak{u} \stackrel{k}{\sim} \tilde{\mathfrak{t}}$ and $\mathfrak{u} \neq \tilde{\mathfrak{t}}$ : Now, $c_{\mathfrak{t}}(k)=c_{\mathfrak{u}}(k+2)$ and $c_{\mathfrak{u}}(k+1)=-c_{\mathfrak{u}}(k)$, so the coefficient of $v_{\mathfrak{u}}$ in $S_{k} E_{k+1} E_{k} v_{\mathfrak{t}}$ is

$$
e_{\mathfrak{t} \tilde{\mathfrak{t}}}(k) e_{\tilde{\mathfrak{t}} \mathfrak{t}}(k+1) s_{\tilde{\mathfrak{t}} \mathfrak{u}}(k)=\frac{e_{\mathfrak{t u}}(k)}{c_{\tilde{\mathfrak{t}}}(k)+c_{\mathfrak{u}}(k)}=e_{\mathfrak{t u}}(k) a_{\mathfrak{u}}(k+1),
$$

which is the coefficient of $v_{\mathfrak{u}}$ in $S_{k+1} E_{k} v_{\mathfrak{t}}$.

Case 3. $\mathfrak{u} \stackrel{k+1}{\sim} \tilde{\mathfrak{t}}$ and $\mathfrak{u} \neq \tilde{\mathfrak{t}}$ : Since $c_{\mathfrak{u}}(k)=-c_{\mathfrak{t}}(k+1)$, the coefficient of $v_{\mathfrak{u}}$ in $S_{k} E_{k+1} E_{k} v_{\mathrm{t}}$ is

$$
a_{\mathfrak{u}}(k) e_{\tilde{\mathfrak{t}} \mathfrak{u}}(k+1) e_{\tilde{s} \mathfrak{t}}(k)=\frac{e_{\tilde{\mathfrak{t}} \mathfrak{u}}(k+1) e_{\tilde{s} \tilde{\mathfrak{t}}}(k)}{c_{\mathfrak{u}}(k+1)+c_{\tilde{\mathfrak{t}}}(k+1)}=e_{\mathfrak{t} \tilde{\mathfrak{t}}}(k) s_{\tilde{\mathfrak{t}} \mathfrak{u}}(k+1),
$$

which is the coefficient of $v_{\mathfrak{u}}$ in $S_{k+1} E_{k} v_{\mathfrak{t}}$.

Now suppose that $S_{k} \mathfrak{u}$ is defined and let $\mathfrak{w}=S_{k+1} S_{k} \mathfrak{u}$ be as above. Then the coefficient of $v_{S_{k} \mathfrak{u}}$ in $S_{k} E_{k+1} E_{k} v_{\mathfrak{t}}$ is

$$
\begin{aligned}
e_{\mathfrak{t} \mathfrak{t}}(k) e_{\tilde{\mathfrak{t}} \mathfrak{u}}(k+1) b_{\mathfrak{u}}(k) & =\sqrt{e_{\mathfrak{t t}}(k)} \sqrt{e_{\mathfrak{u u}}(k+1)} b_{\mathfrak{u}}(k) \\
& =\sqrt{e_{\mathfrak{t t}}(k)} \sqrt{e_{\mathfrak{w} \mathfrak{w}}(k)} b_{\mathfrak{w}}(k+1) \\
& =e_{\mathfrak{t w}}(k) b_{\mathfrak{w}}(k+1)
\end{aligned}
$$

where the second equality comes from (4.12)(f). As $S_{k} \mathfrak{u}=S_{k+1} \mathfrak{w}$ this is the coefficient of $v_{S_{k} \mathfrak{u}}$ in $S_{k+1} E_{k} v_{\mathfrak{t}}$. This completes the proof of (a).

(b) We let the reader work out the expansions of $E_{k+1} E_{k} S_{k+1} v_{\mathfrak{t}}$ and $E_{k+1} S_{k} v_{\mathrm{t}}$. To show that these two expressions are equal there are four cases to consider.

Case 1. $\mathfrak{t}_{k}=\mathfrak{t}_{k+2}$ and $\mathfrak{t}_{k-1}=\mathfrak{t}_{k+1}$ : We have

$$
\begin{aligned}
E_{k+1} E_{k} S_{k+1} v_{\mathfrak{t}} & =E_{k+1} e_{\mathfrak{t t}}(k) s_{\mathfrak{t} \mathfrak{t}}(k+1) v_{\mathfrak{t}}=\frac{1-e_{\mathfrak{t t}}(k)}{2 c_{\mathfrak{t}}(k+1)} E_{k+1} v_{\mathfrak{t}} \\
& =s_{\mathfrak{t t}}(k) E_{k+1} v_{\mathfrak{t}}=E_{k+1} S_{k} v_{\mathfrak{t}}
\end{aligned}
$$


Case 2. $\mathfrak{t}_{k} \neq \mathfrak{t}_{k+2}$ and $\mathfrak{t}_{k-1}=\mathfrak{t}_{k+1}$ : Define $\tilde{\mathfrak{t}} \in \mathscr{T}_{n}^{u d}(\lambda)$ to be the unique updown tableau such that $\tilde{\mathfrak{t}} \stackrel{k}{\sim} \mathfrak{t}$ and $\tilde{\mathfrak{t}}_{k}=\mathfrak{t}_{k+2}$. Then $\tilde{\mathfrak{t}} \neq \mathfrak{t}$ and

$$
E_{k+1} E_{k} S_{k+1} v_{\mathfrak{t}}=a_{\mathfrak{t}}(k+1) e_{\mathfrak{t \mathfrak { t }}}(k) E_{k+1} v_{\mathfrak{t}}=s_{\mathfrak{t \mathfrak { t }}}(k) E_{k+1} v_{\mathfrak{t}}=E_{k+1} S_{k} v_{s},
$$

where the second equality uses the facts that $c_{\mathfrak{t}}(k+1)=-c_{\mathfrak{t}}(k), c_{\mathfrak{t}}(k+2)=$ $c_{\mathfrak{t}}(k)$ and $\left(S_{k+1} \mathfrak{t}\right)_{k-1} \neq\left(S_{k+1} \mathfrak{t}\right)_{k+1}$.

Case 3. $\mathfrak{t}_{k}=\mathfrak{t}_{k+2}$ and $\mathfrak{t}_{k-1} \neq \mathfrak{t}_{k+1}$ : Define $\tilde{\mathfrak{t}} \in \mathscr{T}_{n}^{u d}(\lambda)$ to be the unique updown tableau such that $\tilde{\mathfrak{t}}^{k+1} \mathfrak{t}$ and $\tilde{\mathfrak{t}}_{k+1}=\mathfrak{t}_{k-1}$. Then

$$
\begin{aligned}
E_{k+1} E_{k} S_{k+1} v_{\mathfrak{t}} & =s_{\tilde{\mathfrak{t}}}(k+1) e_{\tilde{\mathfrak{t}} \mathfrak{t}}(k) E_{k+1} v_{\tilde{\mathfrak{t}}} \\
& =\frac{e_{\tilde{\mathfrak{t}}}(k+1) e_{\tilde{\mathfrak{t}}}(k)}{c_{\mathfrak{t}}(k+1)+c_{\tilde{\mathfrak{t}}}(k+1)} \sum_{\substack{k+1 \\
\mathfrak{u}^{k+1}}} e_{\tilde{\mathfrak{t}} \mathfrak{\mathfrak { t }}}(k+1) v_{\mathfrak{u}} \\
& =a_{\mathfrak{t}}(k) \sum_{\tilde{\mathfrak{t}}^{k+1} \sim \mathfrak{u}} e_{\mathfrak{t} \mathfrak{u}}(k+1) v_{\mathfrak{u}}=E_{k+1} S_{k} v_{\mathfrak{t}},
\end{aligned}
$$

where we have used the facts that $c_{\tilde{\mathfrak{t}}}(k+1)=-c_{\mathfrak{t}}(k)$ and $\left(S_{k} \mathfrak{t}\right)_{k} \neq\left(S_{k} \mathfrak{t}\right)_{k+2}$. Case 4. $\mathfrak{t}_{k} \neq \mathfrak{t}_{k+2}$ and $\mathfrak{t}_{k-1} \neq \mathfrak{t}_{k+1}$ : First observe that because of our assumptions we have $E_{k+1} E_{k} S_{k+1} v_{\mathfrak{t}}=b_{\mathfrak{t}}(k+1) E_{k+1} E_{k} v_{S_{k+1} \mathfrak{t}}$ and $E_{k+1} S_{k} v_{\mathfrak{t}}=$ $b_{\mathfrak{t}}(k) E_{k+1} v_{S_{k} \mathfrak{t}}$. If $\left(S_{k+1} \mathfrak{t}\right)_{k-1} \neq\left(S_{k+1} \mathfrak{t}\right)_{k+1}$ then we also have $\left(S_{k} \mathfrak{t}\right)_{k} \neq$ $\left(S_{k} \mathfrak{t}\right)_{k+2}$ so that $E_{k+1} S_{k} S_{k+1} v_{\mathfrak{t}}=0=E_{k+1} S_{k} v_{\mathfrak{t}}$.

Suppose now that $\left(S_{k+1} \mathfrak{t}\right)_{k-1}=\left(S_{k+1} \mathfrak{t}\right)_{k+1}$ and let $\tilde{\mathfrak{t}} \in \mathscr{T}_{n}^{u d}(\lambda)$ be the unique updown tableau such that $\tilde{\mathfrak{t}} \stackrel{k}{\sim} S_{k+1} \mathfrak{t}$ and $\tilde{\mathfrak{t}}_{k}=\mathfrak{t}_{k+2}$. Set $\mathfrak{u}=$ $S_{k} \mathfrak{t}$ and $\mathfrak{w}=S_{k+1} \mathfrak{t}$ and observe that the assumptions of (4.12)(f) hold, so that $b_{\mathfrak{u}}(k) \sqrt{e_{\mathfrak{u} \mathfrak{u}}(k+1)}=b_{\mathfrak{w}}(k+1) \sqrt{e_{\mathfrak{w} \mathfrak{w}}(k)}$. As $b_{\mathfrak{t}}(k)=b_{\mathfrak{u}}(k)$ and $b_{\mathfrak{t}}(k+1)=b_{\mathfrak{w}}(k+1)$, the reader should now have no difficulty in using $(4.12)(\mathrm{d})$, together with the fact that $\mathfrak{u}^{\prime} \stackrel{k+1}{\sim} \tilde{\mathfrak{t}}$ if and only if $\mathfrak{u}^{\prime} \stackrel{k+1}{\sim} S_{k} \mathfrak{t}$, to show that

$$
\begin{aligned}
E_{k+1} E_{k} S_{k+1} v_{\mathfrak{t}} & =b_{\mathfrak{t}}(k+1) \sum_{{\mathfrak{\mathfrak { u } ^ { \prime }}}^{k+1} \sim} e_{\tilde{\mathfrak{t}}, S_{k+1}}(k) e_{\tilde{\mathfrak{t}}, \mathfrak{u}^{\prime}}(k+1) v_{\mathfrak{u}^{\prime}} \\
& =b_{\mathfrak{t}}(k) \sum_{\mathfrak{u}^{\prime} \stackrel{+1}{\sim} S_{k} \mathfrak{t}} e_{S_{k} \mathfrak{t}, \mathfrak{u}^{\prime}}(k+1) v_{\mathfrak{u}^{\prime}}=E_{k+1} S_{k} v_{\mathfrak{t}}
\end{aligned}
$$

The next Proposition shows that the action of $\mathscr{W}_{r, n}(\mathbf{u})$ on $\Delta(\lambda)$ respects the two relations $2.1(\mathrm{~b})(\mathrm{i})$ and $2.1(\mathrm{~d})(\mathrm{i})$. 
Proposition 4.27. Suppose that $\mathfrak{t} \in \mathscr{T}_{n}^{u d}(\lambda)$ and that $|k-l|>1$. Then:

a) $S_{k} S_{l} v_{\mathfrak{t}}=S_{l} S_{k} v_{\mathfrak{t}}$

b) $S_{k} E_{l} v_{\mathrm{t}}=E_{l} S_{k} v_{\mathrm{t}}$.

Proof. We prove only (a) as the proof of part (b) is similar to, but easier than (a).

First suppose that $\mathfrak{t}_{k-1}=\mathfrak{t}_{k+1}$ and $\mathfrak{t}_{l-1}=\mathfrak{t}_{l+1}$. Then

$$
S_{k} S_{l} v_{\mathfrak{t}}=\sum_{\substack{\mathfrak{u} \sim \mathfrak{t}, \mathfrak{w} \sim \\ \sim} \mathfrak{u}} s_{\mathfrak{t u}}(l) s_{\mathfrak{u} \mathfrak{w}}(k) v_{\mathfrak{w}}
$$

Now for each pair of updown tableaux $(\mathfrak{w}, \mathfrak{u})$ with $\mathfrak{w} \stackrel{k}{\sim} \mathfrak{u} \stackrel{l}{\sim} \mathfrak{t}$ there is a unique updown tableau $\mathfrak{u}^{\prime}$ such that $\mathfrak{w} \stackrel{l}{\sim} \mathfrak{u}^{\prime} \stackrel{k}{\sim} \mathfrak{t}$; more precisely, $\mathfrak{u}_{k}^{\prime}=\mathfrak{w}_{k}$ and $\mathfrak{u}_{a}^{\prime}=\mathfrak{t}_{a}$ for $a \neq l$. Notice that $\delta_{\mathfrak{u v w}}=\delta_{\mathfrak{t u} \prime^{\prime}}$ and $\delta_{\mathfrak{t u}}=\delta_{\mathfrak{w} \mathfrak{u}^{\prime}}$. Therefore,

$$
\begin{aligned}
s_{\mathfrak{t u}}(l) s_{\mathfrak{u w}}(k) & =\frac{\sqrt{e_{\mathfrak{t t}}(l)} \sqrt{e_{\mathfrak{u u}}(l)}-\delta_{\mathfrak{t u}}}{c_{\mathfrak{t}}(l)+c_{\mathfrak{u}}(l)} \frac{\sqrt{e_{\mathfrak{u} \mathfrak{u}}(k)} \sqrt{e_{\mathfrak{w} w}(k)}-\delta_{\mathfrak{u w}}}{c_{\mathfrak{u}}(k)+c_{\mathfrak{w}}(k)} \\
& =\frac{\sqrt{e_{\mathfrak{u}^{\prime} \mathfrak{u}^{\prime}}(l)} \sqrt{e_{\mathfrak{w} \mathfrak{w}}(l)}-\delta_{\mathfrak{w} \mathfrak{u}^{\prime}}}{c_{\mathfrak{u}^{\prime}}(l)+c_{\mathfrak{w}}(l)} \frac{\sqrt{e_{\mathfrak{t t}}(k)} \sqrt{e_{\mathfrak{u}^{\prime} \mathfrak{u}^{\prime}}(k)}-\delta_{\mathfrak{u}^{\prime} \mathfrak{t}}}{c_{\mathfrak{t}}(k)+c_{\mathfrak{u}^{\prime}}(k)} \\
& =s_{\mathfrak{u}^{\prime} \mathfrak{w}}(l) s_{\mathfrak{t u}}(k),
\end{aligned}
$$

where the second equality uses (4.8) and (4.12)(e). Hence,

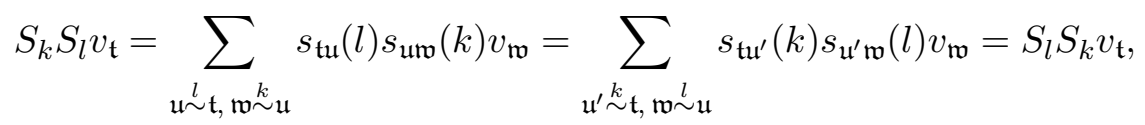

as required.

Assume now that $\mathfrak{t}_{k-1} \neq \mathfrak{t}_{k+1}$ and $\mathfrak{t}_{l-1}=\mathfrak{t}_{l+1}$. Then

$$
\begin{aligned}
& S_{k} S_{l} v_{\mathfrak{t}}=\sum_{\substack{\mathfrak{u} \sim \mathfrak{t}}} s_{\mathfrak{t u}}(l)\left(a_{\mathfrak{u}}(k) v_{\mathfrak{u}}+b_{\mathfrak{u}}(k) v_{S_{k} \mathfrak{u}}\right) \\
& =a_{\mathfrak{t}}(k) \sum_{\substack{\mathfrak{u} \sim \mathfrak{t} \\
l}} s_{\mathfrak{t u}}(l) v_{\mathfrak{u}}+b_{\mathfrak{t}}(k) \sum_{\substack{\mathfrak{u} \sim \mathfrak{t} \\
l}} s_{\mathfrak{t u}}(l) v_{S_{k} \mathfrak{u}} \\
& =a_{\mathfrak{t}}(k) \sum_{\substack{\mathfrak{u} \sim \mathfrak{l}}} s_{\mathfrak{t u}}(l) v_{\mathfrak{u}}+b_{\mathfrak{t}}(k) \sum_{\substack{\mathfrak{u}^{\prime} \\
\sim} S_{k} \mathfrak{t}} s_{S_{k}, \mathfrak{t} \mathfrak{u}^{\prime}}(l) v_{\mathfrak{u}^{\prime}}=S_{l} S_{k} v_{\mathfrak{t}} .
\end{aligned}
$$


Interchanging $k$ and $l$ covers the case when $\mathfrak{t}_{k-1}=\mathfrak{t}_{k+1}$ and $\mathfrak{t}_{l-1} \neq \mathfrak{t}_{l+1}$

Finally, consider the case when $\mathfrak{t}_{k-1} \neq \mathfrak{t}_{k+1}$ and $\mathfrak{t}_{l-1} \neq \mathfrak{t}_{l+1}$. Then

$$
\begin{aligned}
S_{k} S_{l} v_{\mathfrak{t}} & =a_{\mathfrak{t}}(k) a_{\mathfrak{t}}(l) v_{\mathfrak{t}}+a_{S_{l} \mathfrak{t}}(k) b_{\mathfrak{t}}(l) v_{S_{l} \mathfrak{t}}+b_{\mathfrak{t}}(k) a_{\mathfrak{t}}(l) v_{S_{k} \mathfrak{t}}+b_{S_{l} \mathfrak{t}}(k) b_{\mathfrak{t}}(l) v_{S_{k} S_{l} \mathfrak{t}} \\
& =a_{\mathfrak{t}}(l) a_{\mathfrak{t}}(k) v_{\mathfrak{t}}+a_{\mathfrak{t}}(k) b_{\mathfrak{t}}(l) v_{S_{l} \mathfrak{t}}+a_{S_{k}}(l) b_{\mathfrak{t}}(k) v_{S_{k} \mathfrak{t}}+b_{S_{k} \mathfrak{t}}(l) b_{\mathfrak{t}}(k) v_{S_{l} S_{k} \mathfrak{t}},
\end{aligned}
$$

since $a_{S_{l} \mathfrak{t}}(k)=a_{\mathfrak{t}}(k)$ and $a_{\mathfrak{t}}(l)=a_{S_{k} \mathfrak{t}}(l)$, by definition, and $b_{S_{l} \mathfrak{t}}(k)=b_{\mathfrak{t}}(k)$ and $b_{S_{k} \mathfrak{t}}(l)=b_{\mathfrak{t}}(l)$ by $(4.12)(\mathrm{b})$. Hence, $S_{k} S_{l} v_{\mathfrak{t}}=S_{l} S_{k} v_{\mathfrak{t}}$ if $\mathfrak{t}_{k-1} \neq \mathfrak{t}_{k+1}$ and $\mathfrak{t}_{l-1} \neq \mathfrak{t}_{l+1}$. This completes the proof of (a).

Finally, we prove that the action of $\mathscr{W}_{r, n}(\mathbf{u})$ on $\Delta(\lambda)$ respects the braid relations of length three.

LEMMA 4.28. Suppose that $\mathfrak{t} \in \mathscr{T}_{n}^{u d}(\lambda)$ with $\mathfrak{t}_{k-1} \neq \mathfrak{t}_{k+1}$ and $\mathfrak{t}_{k} \neq \mathfrak{t}_{k+2}$, where $1 \leq k<n-1$. Then $S_{k} S_{k+1} S_{k} v_{\mathfrak{t}}=S_{k+1} S_{k} S_{k+1} v_{\mathfrak{t}}$.

Proof. We consider two cases.

Case 1. $S_{k} \mathfrak{t}$ is not defined, or $S_{k} \mathfrak{t}$ is defined and $\left(S_{k} \mathfrak{t}\right)_{k} \neq\left(S_{k} \mathfrak{t}\right)_{k+2}$ : First suppose that $S_{k} \mathfrak{t}$ is defined. If $S_{k+1} \mathfrak{t}$ is defined then $\left(S_{k+1} \mathfrak{t}\right)_{k-1} \neq$ $\left(S_{k+1} \mathfrak{t}\right)_{k+1}$, and if $S_{k+1} S_{k} \mathfrak{t}$ is defined then $\left(S_{k+1} S_{k} \mathfrak{t}\right)_{k-1} \neq\left(S_{k+1} S_{k} \mathfrak{t}\right)_{k+1}$ because $\mathfrak{t}_{k} \neq \mathfrak{t}_{k+2}$. Thus we have

$$
\begin{aligned}
S_{k} S_{k+1} & S_{k} v_{\mathfrak{t}}=\left(a_{\mathfrak{t}}(k)^{2} a_{\mathfrak{t}}(k+1)+b_{\mathfrak{t}}(k) a_{S_{k} \mathfrak{t}}(k+1) b_{S_{k} \mathfrak{t}}(k)\right) v_{\mathfrak{t}} \\
& +\left(a_{\mathfrak{t}}(k) a_{\mathfrak{t}}(k+1) b_{\mathfrak{t}}(k)+b_{\mathfrak{t}}(k) a_{S_{k} \mathfrak{t}}(k+1) a_{S_{k} \mathfrak{t}}(k)\right) v_{S_{k} \mathfrak{t}} \\
& +a_{\mathfrak{t}}(k) b_{\mathfrak{t}}(k+1) a_{S_{k+1} \mathfrak{t}}(k) v_{S_{k+1} \mathfrak{t}}+a_{\mathfrak{t}}(k) b_{\mathfrak{t}}(k+1) b_{S_{k+1} \mathfrak{t}}(k) v_{S_{k} S_{k+1} \mathfrak{t}} \\
& +b_{\mathfrak{t}}(k) b_{S_{k} \mathfrak{t}}(k+1) a_{S_{k+1} S_{k} \mathfrak{t}}(k) v_{S_{k+1} S_{k} \mathfrak{t}} \\
& +b_{\mathfrak{t}}(k) b_{S_{k} \mathfrak{t}}(k+1) b_{S_{k+1} S_{k} \mathfrak{t}}(k) v_{S_{k} S_{k+1} S_{k} \mathfrak{t} .}
\end{aligned}
$$

Now, $\mathfrak{t}_{k-1} \neq \mathfrak{t}_{k+1}$, or if $S_{k} S_{k+1} \mathfrak{t}$ is defined, then $\left(S_{k} S_{k+1} \mathfrak{t}\right)_{k} \neq\left(S_{k} S_{k+1} \mathfrak{t}\right)_{k+2}$. Therefore, we have

$$
\begin{aligned}
S_{k+1} & S_{k} S_{k+1} v_{\mathfrak{t}}=\left(a_{\mathfrak{t}}(k+1)^{2} a_{\mathfrak{t}}(k)+b_{\mathfrak{t}}(k+1) a_{S_{k+1} \mathfrak{t}}(k) b_{S_{k+1} \mathfrak{t}}(k+1)\right) v_{\mathfrak{t}} \\
& +\left(a_{\mathfrak{t}}(k+1) a_{\mathfrak{t}}(k) b_{\mathfrak{t}}(k+1)+b_{\mathfrak{t}}(k+1) a_{S_{k+1}}(k) a_{S_{k+1} \mathfrak{t}}(k+1)\right) v_{S_{k+1} \mathfrak{t}} \\
& +a_{\mathfrak{t}}(k+1) b_{\mathfrak{t}}(k) a_{S_{k} \mathfrak{t}}(k+1) v_{S_{k} \mathfrak{t}}+a_{\mathfrak{t}}(k+1) b_{\mathfrak{t}}(k) b_{S_{k} \mathfrak{t}}(k+1) v_{S_{k+1} S_{k} \mathfrak{t}} \\
& +b_{\mathfrak{t}}(k+1) b_{S_{k+1} \mathfrak{t}}(k) a_{S_{k} S_{k+1} \mathfrak{t}}(k+1) v_{S_{k} S_{k+1} \mathfrak{t}} \\
& +b_{\mathfrak{t}}(k+1) b_{S_{k+1} \mathfrak{t}}(k) b_{S_{k} S_{k+1} \mathfrak{t}}(k+1) v_{S_{k+1} S_{k} S_{k+1} \mathfrak{t} .}
\end{aligned}
$$


Now, $b_{S_{k}}(k)=b_{\mathfrak{t}}(k)$ and $b_{S_{k+1} \mathfrak{t}}(k+1)=b_{\mathfrak{t}}(k+1)$ by $(4.12)(\mathrm{a})$. So, in order to check that the coefficients of $v_{\mathfrak{t}}$ are equal in the last two equations we have to show that

$$
\begin{aligned}
& a_{\mathfrak{t}}(k)^{2} a_{\mathfrak{t}}(k+1)+a_{S_{k} \mathfrak{t}}(k+1)\left(1-a_{\mathfrak{t}}(k)^{2}\right) \\
& \quad=a_{\mathfrak{t}}(k) a_{\mathfrak{t}}(k+1)^{2}+a_{S_{k+1} \mathfrak{t}}(k+1)\left(1-a_{\mathfrak{t}}(k+1)^{2}\right) ;
\end{aligned}
$$

however, this is just a special case of the easy identity

$$
\begin{aligned}
& \frac{1}{(b-a)^{2}(c-b)}+\frac{1}{c-a}\left(1-\frac{1}{(b-a)^{2}}\right) \\
& =\frac{1}{(b-a)(c-b)^{2}}+\frac{1}{c-a}\left(1-\frac{1}{(c-b)^{2}}\right)
\end{aligned}
$$

To see that the coefficients of $v_{S_{k} \mathfrak{t}}$ and $v_{S_{k+1}}$ are equal amounts to the following easily checked identities

$$
\begin{aligned}
a_{S_{k}}(k) a_{S_{k} \mathfrak{t}}(k+1)+a_{\mathfrak{t}}(k) a_{\mathfrak{t}}(k+1) & =a_{\mathfrak{t}}(k+1) a_{S_{k} \mathfrak{t}}(k+1), \\
a_{S_{k+1} \mathfrak{t}}(k) a_{S_{k+1} \mathfrak{t}}(k+1)+a_{\mathfrak{t}}(k) a_{\mathfrak{t}}(k+1) & =a_{\mathfrak{t}}(k) a_{S_{k+1} \mathfrak{t}}(k) .
\end{aligned}
$$

For the coefficients of $v_{S_{k+1} S_{k} \mathfrak{t}}$ and $v_{S_{k} S_{k+1} \mathfrak{t}}$, note that $a_{S_{k+1} S_{k} \mathfrak{t}}(k)=a_{\mathfrak{t}}(k+1)$ and $a_{S_{k} S_{k+1} \mathfrak{t}}(k+1)=a_{\mathfrak{t}}(k)$. Finally, three applications of $(4.12)(\mathrm{c})$ shows that the coefficients in $v_{S_{k} S_{k+1} S_{k} \mathfrak{t}}=v_{S_{k+1} S_{k} S_{k+1}}$ are equal in both equations.

If $S_{k} \mathfrak{t}$ is not defined then $a_{\mathfrak{t}}(k)= \pm 1$ and $b_{\mathfrak{t}}(k)=0$ by Lemma $4.11(\mathrm{~b})$. Hence, the argument above is still valid if we set $b_{\mathfrak{t}}(k)=0$.

Case 2. $S_{k} \mathfrak{t}$ is defined and $\left(S_{k} \mathfrak{t}\right)_{k}=\left(S_{k} \mathfrak{t}\right)_{k+2}$ : If $S_{k+1} \mathfrak{t}$ is defined then $\left(S_{k+1} \mathfrak{t}\right)_{k-1}=\left(S_{k+1} \mathfrak{t}\right)_{k+1}$. Let $\tilde{\mathfrak{t}}$ be the unique updown tableau such that $\tilde{\mathfrak{t}} \stackrel{k+1}{\sim} S_{k} \mathfrak{t}$ and $\tilde{\mathfrak{t}}_{k+1}=\mathfrak{t}_{k-1}$. Observe that if $\mathfrak{u} \stackrel{k+1}{\sim} \tilde{\mathfrak{t}}$ and $\mathfrak{u} \neq \tilde{\mathfrak{t}}$ then $\mathfrak{u}_{k-1} \neq \mathfrak{u}_{k+1}$. Therefore,

$$
\begin{aligned}
& S_{k} S_{k+1} S_{k} v_{\mathfrak{t}}=a_{\mathfrak{t}}(k)^{2} a_{\mathfrak{t}}(k+1) v_{\mathfrak{t}}+a_{\mathfrak{t}}(k) a_{\mathfrak{t}}(k+1) b_{\mathfrak{t}}(k) v_{S_{k} \mathfrak{t}} \\
& +a_{\mathfrak{t}}(k) b_{\mathfrak{t}}(k+1) \sum_{\substack{\mathfrak{u} \sim \tilde{\mathfrak{t}}\\
}} s_{S_{k+1} \mathfrak{t}, \mathfrak{u}}(k) v_{\mathfrak{u}}+b_{\mathfrak{t}}(k) \sum_{\substack{k \\
\mathfrak{u} \sim \mathfrak{t}}} s_{S_{k} \mathfrak{t}, \tilde{\mathfrak{t}}}(k+1) s_{\tilde{\mathfrak{t}} \mathfrak{u}}(k) v_{\mathfrak{u}}
\end{aligned}
$$

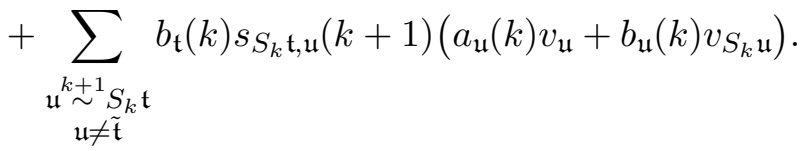


Similarly,

$$
\begin{aligned}
S_{k+1} & S_{k} S_{k+1} v_{\mathfrak{t}}=a_{\mathfrak{t}}(k+1)^{2} a_{\mathfrak{t}}(k) v_{\mathfrak{t}}+a_{\mathfrak{t}}(k+1) a_{\mathfrak{t}}(k) b_{\mathfrak{t}}(k+1) v_{S_{k+1} \mathfrak{t}} \\
& +a_{\mathfrak{t}}(k+1) b_{\mathfrak{t}}(k) \sum_{\mathfrak{k}^{k+1} \tilde{\mathfrak{t}}} s_{S_{k} \mathfrak{t}, \mathfrak{u}}(k+1) v_{\mathfrak{u}} \\
& +b_{\mathfrak{t}}(k+1) \sum_{\substack{k+1 \\
\mathfrak{u} \sim}} s_{S_{k+1} \mathfrak{t}, \tilde{\mathfrak{t}}}(k) s_{\tilde{\mathfrak{t}} \mathfrak{u}}(k+1) v_{\mathfrak{u}} \\
& +\sum_{\substack{\mathfrak{k} \sim S_{k+1} \mathfrak{t} \\
\mathfrak{u} \neq \mathfrak{\mathfrak { t }}}} b_{\mathfrak{t}}(k+1) s_{S_{k+1} \mathfrak{t}, \mathfrak{u}}(k)\left(a_{\mathfrak{u}}(k+1) v_{\mathfrak{u}}+b_{\mathfrak{u}}(k+1) v_{S_{k+1} \mathfrak{u}}\right) .
\end{aligned}
$$

We now compare each of the coefficients in the last two displayed equations.

First we consider the coefficient of $v_{\mathfrak{t}}$. To show that the coefficients of $v_{\mathfrak{t}}$ are equal in the two expressions above, we have to prove that

$$
\begin{aligned}
& a_{\mathfrak{t}}(k)^{2} a_{\mathfrak{t}}(k+1)+b_{\mathfrak{t}}(k) s_{S_{k} \mathfrak{t}, S_{k} \mathfrak{t}}(k+1) b_{S_{k} \mathfrak{t}}(k) \\
& \quad=a_{\mathfrak{t}}(k+1)^{2} a_{\mathfrak{t}}(k)+b_{\mathfrak{t}}(k+1) s_{S_{k+1} \mathfrak{t}, S_{k+1} \mathfrak{t}}(k) b_{S_{k+1} \mathfrak{t}}(k+1) .
\end{aligned}
$$

Now, $b_{\mathfrak{t}}(k)=b_{S_{k} \mathfrak{t}}(k)$ and $b_{\mathfrak{t}}(k+1)=b_{S_{k+1}}(k+1)$ by $(4.12)(\mathrm{a})$. So, the last identity is equivalent to

$$
\begin{aligned}
& a_{\mathfrak{t}}(k)^{2} a_{\mathfrak{t}}(k+1)+\frac{e_{S_{k} \mathfrak{t}, S_{k} \mathfrak{t}}(k+1)-1}{2 c_{S_{k} \mathfrak{t}}(k+1)} b_{S_{k} \mathfrak{t}}(k)^{2} \\
& =a_{\mathfrak{t}}(k+1)^{2} a_{\mathfrak{t}}(k)+\frac{e_{S_{k+1} \mathfrak{t}, S_{k+1} \mathfrak{t}}(k)-1}{2 c_{S_{k+1} \mathfrak{t}}(k)} b_{S_{k+1} \mathfrak{t}}(k+1)^{2} .
\end{aligned}
$$

This equation is easily verified using the definitions and Lemma 4.20. Hence, the coefficients of $v_{\mathfrak{t}}$ in $S_{k} S_{k+1} S_{k} v_{\mathfrak{t}}$ and $S_{k+1} S_{k} S_{k+1}$ are equal.

Now consider the coefficient of $v_{S_{k} \mathfrak{t}}$ in both equations. Since $a_{S_{k}} \mathfrak{t}(k)-$ $a_{\mathfrak{t}}(k+1)=2 c_{S_{k} \mathfrak{t}}(k+1) /\left(c_{\mathfrak{t}}(k)+c_{S_{k+1} \mathfrak{t}}(k)\right)\left(c_{\mathfrak{t}}(k)+c_{S_{k} \mathfrak{t}}(k)\right)$, we see that

$$
\begin{aligned}
& s_{S_{k}} \mathfrak{t}, S_{k} \mathfrak{t}(k+1)\left(a_{S_{k} \mathfrak{t}}(k)-a_{\mathfrak{t}}(k+1)\right) b_{\mathfrak{t}}(k)+a_{\mathfrak{t}}(k) a_{\mathfrak{t}}(k+1) b_{\mathfrak{t}}(k) \\
& =e_{S_{k} \mathfrak{t}, S_{k} \mathfrak{t}}(k+1) a_{\mathfrak{t}}(k) a_{\mathfrak{t}}(k+1) b_{\mathfrak{t}}(k) \\
& =\frac{b_{S_{k} \mathfrak{t}}(k) e_{S_{k} \mathfrak{t}, S_{k} \mathfrak{t}}(k+1)}{\left(c_{\tilde{\mathfrak{t}}}(k)+c_{S_{k+1} \mathfrak{t}}(k)\right)\left(c_{\tilde{\mathfrak{t}}}(k+1)+c_{S_{k} \mathfrak{t}}(k+1)\right)} \\
& =\frac{b_{S_{k+1} \mathfrak{t}}(k+1) \sqrt{e_{S_{k+1} \mathfrak{t}, S_{k+1} \mathfrak{t}}(k)} \sqrt{e_{S_{k} \mathfrak{t}, S_{k} \mathfrak{t}}(k+1)}}{\left(c_{\tilde{\mathfrak{t}}}(k)+c_{S_{k+1} \mathfrak{t}}(k)\right)\left(c_{\tilde{\mathfrak{t}}}(k+1)+c_{S_{k} \mathfrak{t}}(k+1)\right)} \\
& =b_{\mathfrak{t}}(k+1) s_{S_{k+1} \mathfrak{t}, \tilde{\mathfrak{t}}}(k) s_{\tilde{\mathfrak{t}}, S_{k} \mathfrak{t}}(k+1) .
\end{aligned}
$$


where the second last equality uses (4.12)(f). Consequently,

$$
\begin{aligned}
& a_{\mathfrak{t}}(k) a_{\mathfrak{t}}(k+1) b_{\mathfrak{t}}(k)+b_{\mathfrak{t}}(k) s_{S_{k} \mathfrak{t}, S_{k} \mathfrak{t}}(k+1) a_{S_{k} \mathfrak{t}}(k) \\
& \quad=a_{\mathfrak{t}}(k+1) b_{\mathfrak{t}}(k) s_{S_{k} \mathfrak{t}, S_{k} \mathfrak{t}}(k+1)+b_{\mathfrak{t}}(k+1) s_{S_{k+1} \mathfrak{t}, \tilde{\mathfrak{t}}}(k) s_{\mathfrak{t}, S_{k}}(k+1) .
\end{aligned}
$$

Hence, the coefficients of $v_{S_{k} \mathfrak{t}}$ in $S_{k} S_{k+1} S_{k} v_{\mathfrak{t}}$ and $S_{k+1} S_{k} S_{k+1} v_{\mathfrak{t}}$ are equal. A similar argument shows that

$$
\begin{aligned}
& a_{\mathfrak{t}}(k) b_{\mathfrak{t}}(k+1) s_{S_{k+1} \mathfrak{t}, S_{k+1} \mathfrak{t}}(k)+b_{\mathfrak{t}}(k) s_{S_{k} \mathfrak{t}, \tilde{\mathfrak{t}}}(k+1) s_{\mathfrak{t}, S_{k+1}}(k) \\
& \quad=a_{\mathfrak{t}}(k+1) a_{\mathfrak{t}}(k) b_{\mathfrak{t}}(k+1)+b_{\mathfrak{t}}(k+1) s_{S_{k+1} \mathfrak{t}, S_{k+1} \mathfrak{t}}(k) a_{S_{k+1} \mathfrak{t}}(k+1) .
\end{aligned}
$$

This proves that the coefficient of $v_{S_{k+1}}$ in $S_{k} S_{k+1} S_{k} v_{\mathfrak{t}}$ and $S_{k+1} S_{k} S_{k+1} v_{\mathfrak{t}}$ are equal.

Now consider the coefficient of $v_{\mathfrak{u}}$ where $\mathfrak{u} \stackrel{k}{\sim} \tilde{\mathfrak{t}}$ and $\mathfrak{u} \notin\left\{\tilde{\mathfrak{t}}, S_{k+1} \mathfrak{t}\right\}$. This time

$$
a_{\mathfrak{u}}(k+1)-a_{\mathfrak{t}}(k)=\frac{c_{S_{k+1} \mathfrak{t}}(k)+c_{\mathfrak{u}}(k)}{\left(c_{S_{k} \mathfrak{t}}(k+1)+c_{\tilde{\mathfrak{t}}}(k+1)\right)\left(c_{\mathfrak{\mathfrak { t }}}(k)+c_{\mathfrak{u}}(k)\right)} .
$$

An argument similar to that for $v_{S_{k}}$ now shows that

$$
b_{\mathfrak{t}}(k) s_{S_{k} \mathfrak{t}, \tilde{\mathfrak{t}}}(k+1) s_{\tilde{\mathfrak{t}} \mathfrak{u}}(k)=b_{\mathfrak{t}}(k+1) s_{S_{k+1} \mathfrak{t}, \mathfrak{u}}(k)\left(a_{\mathfrak{u}}(k+1)-a_{\mathfrak{t}}(k)\right) .
$$

Therefore, the coefficients of $v_{\mathfrak{u}}$ for such $\mathfrak{u}$ in $S_{k} S_{k+1} S_{k} v_{\mathfrak{t}}$ and $S_{k+1} S_{k} S_{k+1} v_{\mathfrak{t}}$ are equal.

Another variation of this argument shows that if $\mathfrak{u} \stackrel{k+1}{\sim} S_{k} \mathfrak{t}$ and $\mathfrak{u} \notin$ $\left\{\tilde{\mathfrak{t}}, S_{k} \mathfrak{t}\right\}$ then the coefficients of $v_{\mathfrak{u}}$ in $S_{k} S_{k+1} S_{k} v_{\mathfrak{t}}$ and $S_{k+1} S_{k} S_{k+1} v_{\mathfrak{t}}$ are both equal.

Next, we suppose that $S_{k} \mathfrak{u}$ is defined and we compare the coefficients of $v_{S_{k} \mathfrak{u}}$ in $S_{k} S_{k+1} S_{k} v_{\mathfrak{t}}$ and $S_{k+1} S_{k} S_{k+1} v_{\mathfrak{t}}$, when $\mathfrak{u} \stackrel{k+1}{\sim} S_{k} \mathfrak{t}$ and $\mathfrak{u} \notin\left\{\tilde{\mathfrak{t}}, S_{k} \mathfrak{t}\right\}$. As $S_{k} \mathfrak{t}$ is defined, $\mathfrak{w}=S_{k+1} S_{k} \mathfrak{u}$ is defined and $\mathfrak{w} \stackrel{k}{\sim} S_{k+1} \mathfrak{t}$ with $\mathfrak{w} \notin\left\{\tilde{\mathfrak{t}}, S_{k+1} \mathfrak{t}\right\}$. Conversely, if $S_{k+1} \mathfrak{w}$ is defined for such $\mathfrak{w}$ then $\mathfrak{u}=S_{k} S_{k+1} \mathfrak{w}$ is defined. Applying (4.12)(f) twice, we have

$$
\begin{aligned}
& b_{\mathfrak{t}}(k) b_{\mathfrak{u}}(k) \sqrt{e_{S_{k} \mathfrak{t}, S_{k} \mathfrak{t}}(k+1)} \sqrt{e_{\mathfrak{u} \mathfrak{u}}(k+1)} \\
& =b_{\mathfrak{t}}(k+1) b_{\mathfrak{w}}(k+1) \sqrt{e_{S_{k+1} \mathfrak{t}, S_{k+1} \mathfrak{t}}(k)} \sqrt{e_{\mathfrak{w} \mathfrak{w}}(k)} .
\end{aligned}
$$

Consequently, because $c_{S_{k} \mathfrak{t}}(k+1)+c_{\mathfrak{u}}(k+1)=c_{\mathfrak{w}}(k)+c_{S_{k+1} \mathfrak{t}}(k)$, we have

$$
b_{\mathfrak{t}}(k) s_{S_{k} \mathfrak{t}, \mathfrak{u}}(k+1) b_{\mathfrak{u}}(k)=b_{\mathfrak{t}}(k+1) s_{S_{k+1} \mathfrak{t}, \mathfrak{w}}(k) b_{\mathfrak{w}}(k+1) .
$$


That is, the coefficients of $v_{S_{k} \mathfrak{u}}$ in $S_{k+1} S_{k} S_{k+1} v_{\mathfrak{t}}$ and $S_{k} S_{k+1} S_{k} v_{\mathfrak{t}}$ are equal.

It remains to compare the coefficients of $v_{\tilde{\mathfrak{t}}}$ in the two equations. To show that these two coefficients are equal we have to prove that

$$
\begin{aligned}
& a_{\mathfrak{t}}(k) b_{\mathfrak{t}}(k+1) s_{S_{k+1} \mathfrak{t}, \tilde{\mathfrak{t}}}(k)+b_{\mathfrak{t}}(k) s_{S_{k} \mathfrak{t}, \tilde{\mathfrak{t}}}(k+1) s_{\tilde{\mathfrak{t}} \tilde{\mathfrak{t}}}(k) \\
& \quad=a_{\mathfrak{t}}(k+1) b_{\mathfrak{t}}(k) s_{S_{k} \mathfrak{t}, \tilde{\mathfrak{t}}}(k+1)+b_{\mathfrak{t}}(k+1) s_{S_{k+1} \mathfrak{t}, \tilde{\mathfrak{t}}}(k) s_{\tilde{\mathfrak{t}}}(k+1) .
\end{aligned}
$$

First note that, by the definitions and (4.12)(a),

$$
\begin{aligned}
b_{\mathfrak{t}}(k+1) s_{S_{k+1} \mathfrak{t}, \tilde{\mathfrak{t}}}(k) & =\frac{b_{\mathfrak{t}}(k+1) \sqrt{e_{S_{k+1} \mathfrak{t}, S_{k+1}}(k)} \sqrt{e_{\tilde{\mathfrak{t}}, \tilde{\mathfrak{t}}}(k)}}{c_{S_{k+1} \mathfrak{t}}(k)+c_{\tilde{\mathfrak{t}}}(k)} \\
& =\frac{b_{\mathfrak{t}}(k) \sqrt{e_{S_{k+1} \mathfrak{t}, S_{k+1} \mathfrak{t}}(k)} \sqrt{e_{\tilde{\mathfrak{t}}, \tilde{\mathfrak{t}}}(k+1)}}{c_{S_{k+1} \mathfrak{t}}(k)+c_{\tilde{\mathfrak{t}}}(k)} \\
& =b_{\mathfrak{t}}(k) s_{S_{k} \mathfrak{t}, \tilde{\mathfrak{t}}}(k+1) \frac{c_{S_{k} \mathfrak{t}}(k+1)+c_{\tilde{\mathfrak{t}}}(k+1)}{c_{S_{k+1} \mathfrak{t}}(k)+c_{\tilde{\mathfrak{t}}}(k)} e_{\tilde{\mathfrak{t}} \mathfrak{t}}(k) .
\end{aligned}
$$

So, it is enough to show that

$$
\begin{aligned}
& \left(c_{S_{k} \mathfrak{t}}(k+1)+c_{\tilde{\mathfrak{t}}}(k+1)\right) e_{\tilde{\mathfrak{t}} \mathfrak{t}}(k)\left(a_{\mathfrak{t}}(k)-s_{\tilde{\mathfrak{t}}}(k+1)\right) \\
& =\left(c_{S_{k+1}}(k)+c_{\tilde{\mathfrak{t}}}(k)\right)\left(a_{\mathfrak{t}}(k+1)-s_{\tilde{\mathfrak{t}}}(k)\right) ;
\end{aligned}
$$

however, this follows from Lemma 4.19. Hence, the coefficients of $v_{\tilde{\mathfrak{t}}}$ in $S_{k+1} S_{k} S_{k+1} v_{\mathfrak{t}}$ and $S_{k} S_{k+1} S_{k} v_{\mathfrak{t}}$ are equal.

This completes the proof of Lemma 4.28.

LEMMA 4.29. Suppose that $\mathfrak{t} \in \mathscr{T}_{n}^{u d}(\lambda)$ and that either $\mathfrak{t}_{k-1}=\mathfrak{t}_{k+1}$ and $\mathfrak{t}_{k} \neq \mathfrak{t}_{k+2}$, or $\mathfrak{t}_{k-1} \neq \mathfrak{t}_{k+1}$ and $\mathfrak{t}_{k}=\mathfrak{t}_{k+2}$, for $1 \leq k<n-1$. Then $S_{k} S_{k+1} S_{k} v_{\mathfrak{t}}=S_{k+1} S_{k} S_{k+1} v_{\mathfrak{t}}$.

Proof. There are again two cases to consider.

Case 1. $S_{k+1} \mathfrak{t}$ is defined: Suppose first that $\mathfrak{t}_{k-1}=\mathfrak{t}_{k+1}$ and $\mathfrak{t}_{k} \neq$ $\mathfrak{t}_{k+2}$. Then $\mathfrak{u}=S_{k+1} \mathfrak{t} \in \mathscr{T}^{u d}(\lambda)$ is well-defined. Furthermore, $\mathfrak{u}_{k} \neq \mathfrak{u}_{k+2}$ and $\mathfrak{u}_{k-1} \neq \mathfrak{u}_{k+1}$, so $S_{k} S_{k+1} S_{k} v_{\mathfrak{u}}=S_{k+1} S_{k} S_{k+1} v_{\mathfrak{u}}$ by Lemma 4.28. Now, $S_{k+1} v_{\mathfrak{u}}=a_{\mathfrak{u}}(k+1) v_{\mathfrak{u}}+b_{\mathfrak{u}}(k+1) v_{\mathfrak{t}}$ and $b_{\mathfrak{u}}(k+1) \neq 0$. Therefore

$$
\begin{aligned}
S_{k} S_{k+1} S_{k} v_{\mathfrak{t}} & =\frac{1}{b_{\mathfrak{u}}(k+1)} S_{k} S_{k+1} S_{k}\left(S_{k+1} v_{\mathfrak{u}}-a_{\mathfrak{u}}(k+1) v_{\mathfrak{u}}\right) \\
& =\frac{1}{b_{\mathfrak{u}}(k+1)}\left(S_{k}\left(S_{k+1} S_{k} S_{k+1}\right) v_{\mathfrak{u}}-a_{\mathfrak{u}}(k+1)\left(S_{k} S_{k+1} S_{k}\right) v_{\mathfrak{u}}\right) \\
& =\frac{1}{b_{\mathfrak{u}}(k+1)}\left(S_{k}\left(S_{k} S_{k+1} S_{k}\right) v_{\mathfrak{u}}-a_{\mathfrak{u}}(k+1)\left(S_{k+1} S_{k} S_{k+1}\right) v_{\mathfrak{u}}\right)
\end{aligned}
$$


by Lemma 4.28. Hence, using Lemma 4.24 twice,

$$
\begin{aligned}
S_{k} S_{k+1} S_{k} v_{\mathfrak{t}} & =\frac{1}{b_{\mathfrak{u}}(k+1)}\left(S_{k+1} S_{k} v_{\mathfrak{u}}-a_{\mathfrak{u}}(k+1)\left(S_{k+1} S_{k} S_{k+1}\right) v_{\mathfrak{u}}\right) \\
& =\frac{1}{b_{\mathfrak{u}}(k+1)}\left(S_{k+1} S_{k}\left(S_{k+1} S_{k+1}\right) v_{\mathfrak{u}}-a_{\mathfrak{u}}(k+1)\left(S_{k+1} S_{k} S_{k+1}\right) v_{\mathfrak{u}}\right) \\
& =\frac{1}{b_{\mathfrak{u}}(k+1)}\left(S_{k+1} S_{k} S_{k+1}\right)\left(S_{k+1} v_{\mathfrak{u}}-a_{\mathfrak{u}}(k+1) v_{\mathfrak{u}}\right) \\
& =\left(S_{k+1} S_{k} S_{k+1}\right) v_{\mathfrak{t}}
\end{aligned}
$$

as required.

The case when $\mathfrak{t}_{k-1} \neq \mathfrak{t}_{k+1}$ and $\mathfrak{t}_{k}=\mathfrak{t}_{k+2}$ can be proved similarly.

Case 2. $S_{k+1} \mathrm{t}$ is not defined: This is equivalent to saying that the two nodes $\mathfrak{t}_{k+2} \ominus \mathfrak{t}_{k+1}$ and $\mathfrak{t}_{k+1} \ominus \mathfrak{t}_{k}$ are in the same row or in the same column. Therefore, either $\mathfrak{t}_{k} \subset \mathfrak{t}_{k+1} \subset \mathfrak{t}_{k+2}$ or $\mathfrak{t}_{k} \supset \mathfrak{t}_{k+1} \supset \mathfrak{t}_{k+2}$. Note that in either case $\mathfrak{t}_{k-1}=\mathfrak{t}_{k+1}$, so we have

$$
E_{k} v_{\mathfrak{t}}=\sum_{\substack{\mathfrak{l} \sim \mathfrak{t} \\ \mathfrak{u} \neq \mathfrak{t}}} e_{\mathfrak{t} \mathfrak{u}}(k) v_{\mathfrak{u}}+e_{\mathfrak{t} \mathfrak{t}}(k) v_{\mathfrak{t}}
$$

By Proposition 4.26 and Proposition 4.25, $S_{k} S_{k+1} S_{k} E_{k} v_{\mathfrak{t}}=S_{k} S_{k+1} E_{k} v_{\mathfrak{t}}=$ $E_{k+1} E_{k} v_{\mathfrak{t}}$ and $S_{k+1} S_{k} S_{k+1} E_{k} v_{\mathfrak{t}}=S_{k+1} E_{k+1} E_{k} v_{\mathfrak{t}}=E_{k+1} E_{k} v_{\mathfrak{t}}$.

Suppose that $\mathfrak{u} \stackrel{k}{\sim} \mathfrak{t}$ and $\mathfrak{u} \neq \mathfrak{t}$. Then $S_{k+1} \mathfrak{u}$ is well-defined and $\mathfrak{u}_{k-1}=\mathfrak{u}_{k+1}$-indeed, the two boxes $\mathfrak{t}_{k+2} \ominus \mathfrak{t}_{k+1}$ and $\mathfrak{t}_{k+1} \ominus \mathfrak{u}_{k}$ belong to different rows and columns. Hence, by Case 1, $S_{k+1} S_{k} S_{k+1} v_{\mathfrak{u}}=S_{k} S_{k+1} S_{k} v_{\mathfrak{u}}$. Consequently, $S_{k+1} S_{k} S_{k+1} e_{\mathfrak{t t}}(k) v_{\mathfrak{t}}=S_{k} S_{k+1} S_{k} e_{\mathfrak{t t}}(k) v_{\mathfrak{t}}$. Cancelling the nonzero factor $e_{\mathfrak{t t}}(k)$ shows that $S_{k} S_{k+1} S_{k} v_{\mathfrak{t}}=S_{k+1} S_{k} S_{k+1} v_{\mathfrak{t}}$.

Proposition 4.30. Suppose that $1 \leq k<n-1$ and $\mathfrak{t} \in \mathscr{T}_{n}^{u d}(\lambda)$. Then $S_{k} S_{k+1} S_{k} v_{\mathfrak{t}}=S_{k+1} S_{k} S_{k+1} v_{\mathfrak{t}}$

Proof. By Lemma 4.28 and Lemma 4.29 it only remains to consider the case when $\mathfrak{t}_{k-1}=\mathfrak{t}_{k+1}$ and $\mathfrak{t}_{k}=\mathfrak{t}_{k+2}$. By Lemma 4.24, Proposition 4.25 and Proposition 4.26(a), we have

$$
S_{k+1} S_{k} S_{k+1} E_{k} v_{\mathfrak{t}}=S_{k+1} S_{k} \cdot S_{k} E_{k+1} E_{k} v_{\mathfrak{t}}=S_{k+1} E_{k+1} E_{k} v_{\mathfrak{t}}=E_{k+1} E_{k} v_{\mathfrak{t}}
$$

on the one hand. Similarly, we also have

$$
S_{k} S_{k+1} S_{k} E_{k} v_{\mathfrak{t}}=S_{k} S_{k+1} E_{k} v_{\mathfrak{t}}=S_{k} \cdot S_{k} E_{k+1} E_{k} v_{\mathfrak{t}}=E_{k+1} E_{k} v_{\mathfrak{t}} .
$$


Therefore, recalling the definition of $E_{k} v_{\mathfrak{t}}$, we have

$$
\left(S_{k+1} S_{k} S_{k+1}-S_{k} S_{k+1} S_{k}\right)\left(e_{\mathfrak{t t}}(k) v_{\mathfrak{t}}+\sum_{\substack{k \\ \mathfrak{u} \sim \mathfrak{t}, \mathfrak{u} \neq \mathfrak{t}}} e_{\mathfrak{t} \mathfrak{u}}(k) v_{\mathfrak{u}}\right)=0 .
$$

Now, if $\mathfrak{u} \stackrel{k}{\sim} \mathfrak{t}$ and $\mathfrak{u} \neq \mathfrak{t}$ then $S_{k} S_{k+1} S_{k} v_{\mathfrak{u}}=S_{k+1} S_{k} S_{k+1} v_{\mathfrak{u}}$ by Lemma 4.29. Consequently, $S_{k} S_{k+1} S_{k} v_{\mathfrak{t}}=S_{k+1} S_{k} S_{k+1} v_{\mathfrak{t}}$ since $e_{\mathfrak{t} t}(k) \neq 0$. This completes the proof.

Proof of Theorem 4.13. The results from Lemma 4.22 to Proposition 4.30 show that the action of the generators of $\mathscr{W}_{r, n}(\mathbf{u})$ on $\Delta(\lambda)$ respects all of the relations of $\mathscr{W}_{r, n}(\mathbf{u})$. Hence, $\Delta(\lambda)$ is a $\mathscr{W}_{r, n}(\mathbf{u})$-module, as we wanted to show.

\section{§5. Irreducible representations and Theorem A}

In this section we use the seminormal representations to show that the cyclotomic Nazarov-Wenzl algebras are always free of rank $r^{n}(2 n-1)$ !!. Before we can do this we need to recall some identities involving updown tableaux.

First, if $\lambda$ is a multipartition of $n-2 m$ let $f^{(n, \lambda)}$ be the number of $n$-updown $\lambda$-tableaux. So, in particular, $f^{(|\lambda|, \lambda)}=\# \mathscr{T}^{s t d}(\lambda)$ is the number of standard $\lambda$-tableaux. Sundaram [Sun86, Lemma 8.7] has given a combinatorial bijection to show that if $\tau$ is a partition ( $s 0 r=1$ ) then the number of $n$-updown $\tau$-tableaux is equal to $\left(\begin{array}{c}n \\ |\tau|\end{array}\right)(n-|\tau|-1) ! ! f^{(|\tau|, \tau)}$. Terada [Ter01] has given a geometric version of this bijection when $|\tau|=0$ and $n$ is even.

LEMmA 5.1. Suppose that $0 \leq m \leq\left\lfloor\frac{n}{2}\right\rfloor$ and that $\lambda \in \Lambda_{r}^{+}(n-2 m)$. Then

$$
f^{(n, \lambda)}=r^{m}\left(\begin{array}{c}
n \\
2 m
\end{array}\right)(2 m-1) ! ! \# \mathscr{T}^{s t d}(\lambda) .
$$

Proof. Using Sundaram's formula from above we have

$$
\begin{aligned}
f^{(n, \lambda)}= & \sum_{\substack{n_{1}, \ldots, n_{r} \\
n_{1}+\cdots+n_{r}=n \\
n_{t}-\left|\lambda^{(t)}\right| \in 2 \mathbb{Z}}}\left(\begin{array}{c}
n \\
n_{1}, \ldots, n_{r}
\end{array}\right) \prod_{t=1}^{r}\left(\begin{array}{c}
n_{t} \\
\left|\lambda^{(t)}\right|
\end{array}\right)\left(n_{t}-\left|\lambda^{(t)}\right|-1\right) ! ! f^{\left(\left|\lambda^{(t)}\right|, \lambda^{(t)}\right)} \\
= & \sum_{\substack{n_{1}, \ldots, n_{r} \\
n_{1}+\cdots+n_{r}=n \\
n_{t}-\left|\lambda^{(t)}\right| \in 2 \mathbb{Z}}} n ! \prod_{t=1}^{r} \frac{\left(n_{t}-\left|\lambda^{(t)}\right|-1\right) ! ! f^{\left(\left|\lambda^{(t)}\right|, \lambda^{(t)}\right)}}{\left(n_{t}-\left|\lambda^{(t)}\right|\right) !\left|\lambda^{(t)}\right| !}
\end{aligned}
$$




$$
\begin{aligned}
& =n ! \prod_{t=1}^{r} \frac{f^{\left(\left|\lambda^{(t)}\right|, \lambda^{(t)}\right)}}{\left|\lambda^{(t)}\right| !} \sum_{\begin{array}{c}
n_{1}, \ldots, n_{r} \\
n_{1}+\cdots+n_{r}=n
\end{array}} \prod_{t=1}^{r} \frac{\left(n_{t}-\left|\lambda^{(t)}\right|-1\right) ! !}{\left(n_{t}-\left|\lambda^{(t)}\right|\right) !} \\
& =\frac{n !}{(n-2 m) !} \# \mathscr{T}^{s t d}(\lambda) \sum_{\substack{a_{1}, \ldots, a_{r} \\
a_{1}+\cdots+a_{r}=m}} \prod_{t=1}^{r} \frac{\left(2 a_{t}-1\right) ! !}{\left(2 a_{t}\right) !}
\end{aligned}
$$

where the summation is now over $a_{t}=\frac{n_{t}-\left|\lambda^{(t)}\right|}{2}$, for $1 \leq t \leq r$. Hence

$$
\begin{aligned}
f^{(n, \lambda)} & =\frac{n !}{(n-2 m) !} \# \mathscr{T}^{s t d}(\lambda) 2^{-m} \sum_{\begin{array}{c}
a_{1}, \ldots, a_{r} \\
a_{1}+\cdots+a_{r}=m
\end{array}} \prod_{t=1}^{r} \frac{1}{a_{t} !} \\
& =\frac{n !}{(n-2 m) !} \# \mathscr{T}^{s t d}(\lambda) \frac{r^{m}}{2^{m} m !}=r^{m}\left(\begin{array}{c}
n \\
2 m
\end{array}\right)(2 m-1) ! ! \# \mathscr{T}^{s t d}(\lambda) .
\end{aligned}
$$

It is well-known from the representation theory of the degenerate Hecke algebras $\mathscr{H}_{r, k}$ that $\sum_{\lambda} \# \mathscr{T}^{s t d}(\lambda)^{2}=r^{k} k$ !, where in the sum $\lambda \in \Lambda_{r}^{+}(k)$.

Corollary 5.2. Suppose that $n \geq 1$ and $r \geq 1$. Then

$$
\sum_{m=0}^{\left\lfloor\frac{n}{2}\right\rfloor} \sum_{\lambda \vdash n-2 m} f^{(n, \lambda)^{2}}=r^{n}(2 n-1) ! ! .
$$

Proof. Using the Lemma we have

$$
\begin{aligned}
\sum_{m=0}^{\left\lfloor\frac{n}{2}\right\rfloor} \sum_{\lambda \vdash n-2 m} f^{(n, \lambda)^{2}} & =\sum_{m=0}^{\left\lfloor\frac{n}{2}\right\rfloor} \sum_{\lambda \vdash n-2 m}\left\{r^{m}\left(\begin{array}{c}
n \\
2 m
\end{array}\right)(2 m-1) ! ! \# \mathscr{T}^{s t d}(\lambda)\right\}^{2} \\
& =\sum_{m=0}^{\left\lfloor\frac{n}{2}\right\rfloor} r^{2 m}\left(\begin{array}{c}
n \\
2 m
\end{array}\right)^{2}((2 m-1) ! !)^{2} \sum_{\lambda \vdash n-2 m} \# \mathscr{T}^{s t d}(\lambda)^{2} \\
& =\sum_{m=0}^{\left\lfloor\frac{n}{2}\right\rfloor} r^{2 m}\left(\begin{array}{c}
n \\
2 m
\end{array}\right)^{2}((2 m-1) ! !)^{2} r^{n-2 m}(n-2 m) ! \\
& =r^{n} \sum_{m=0}^{\left\lfloor\frac{n}{2}\right\rfloor}\left(\begin{array}{c}
n \\
2 m
\end{array}\right)^{2}((2 m-1) ! !)^{2}(n-2 m) ! .
\end{aligned}
$$


To complete the proof, notice that the sum on the right hand side does not depend on $r$, so we can set $r=1$ and deduce the result from the representation theory of the Brauer algebras.

A representation theoretic proof of this result is given in [RY04] where it is obtained as a consequence of the branching rules for the cyclotomic Brauer algebra. The cell modules of the cyclotomic Brauer algebras are indexed by the multipartitions of $n-2 m$, for $0 \leq m \leq\left\lfloor\frac{n}{2}\right\rfloor$. The branching rule [RY04, Theorem 6.1] shows that the dimension of the cell module indexed by $\lambda$ is $f^{(n, \lambda)}$. On the other hand, the cellular basis of the cyclotomic Brauer algebras constructed in [RY04, Theorem 5.11] contains $r^{n}(2 n-1)$ !! elements. Combining these two facts proves the result.

Given two multipartitions $\lambda$ and $\mu$ such that $\mu$ is obtained by adding a box to $\lambda$ we write $\lambda \rightarrow \mu$, or $\mu \leftarrow \lambda$.

TheOREm 5.3. Suppose that $R$ is a field with char $R>2 n$ and the root conditions (Assumption 4.12) hold in $R$. Assume that the parameters $u_{1}, \ldots, u_{r}$ are generic for $\mathscr{W}_{r, n}(\mathbf{u})$ and that $\Omega$ is $\mathbf{u}$-admissible. Then:

a) Suppose $n>1$. There is a $\mathscr{W}_{r, n-1}(\mathbf{u})$-module isomorphism

$$
\Delta(\lambda) \downarrow=\bigoplus_{\substack{\mu \rightarrow \lambda \\ \mu \rightarrow \lambda}} \Delta(\mu) \oplus \bigoplus_{\substack{\nu \\ \lambda \rightarrow \nu}} \Delta(\nu) .
$$

where $\Delta(\lambda) \downarrow$ is $\Delta(\lambda)$ considered as a $\mathscr{W}_{r, n-1}(\mathbf{u})$-module.

b) The seminormal representation $\Delta(\lambda)$ is an irreducible $\mathscr{W}_{r, n}(\mathbf{u})$-module for each multipartition $\lambda$ of $n-2 m$, where $0 \leq m \leq\left\lfloor\frac{n}{2}\right\rfloor$.

c) The set $\left\{\Delta(\lambda) \mid \lambda \vdash n-2 m, 0 \leq m \leq\left\lfloor\frac{n}{2}\right\rfloor\right\}$ is a complete set of irreducible $\mathscr{W}_{r, n}(\mathbf{u})$-modules.

d) $\mathscr{W}_{r, n}(\mathbf{u})$ is a split semisimple $R$-algebra of dimension $r^{n}(2 n-1) !$ !.

Proof. Part (a) follows if we define $\Delta(\mu)$ to be the vector subspace spanned by $v_{\mathfrak{u}}$ with $\mathfrak{u} \in \mathscr{T}_{n}^{u d}(\lambda)$ and $\mathfrak{u}_{n-1}=\mu$.

Let $\mathscr{X}=\left\langle X_{1}, \ldots, X_{n}\right\rangle$. Since $X_{k} v_{\mathfrak{t}}=c_{\mathfrak{t}}(k) v_{\mathfrak{t}}$, for all $\mathfrak{t} \in \mathscr{T}_{n}^{u d}(\lambda)$ and $1 \leq k \leq n$, the seminormal representation $\Delta(\lambda)=\bigoplus_{\mathfrak{t} \in \mathscr{T}_{n}^{u d}(\lambda)} R v_{\mathfrak{t}}$ decomposes into a direct sum of one dimensional submodules as an $\mathscr{X}$ module. Further, by Lemma 4.4(a), this decomposition is multiplicity free. In particular, $\Delta(\lambda) \cong \Delta(\mu)$ if and only if $\lambda=\mu$. Further, if $M$ is a $\mathscr{W}_{r, n}(\mathbf{u})$ submodule of $\Delta(\lambda)$ then $M$ is spanned by some subset of $\left\{v_{\mathfrak{t}} \mid \mathfrak{t} \in \mathscr{T}_{n}^{u d}(\lambda)\right\}$. 
To prove (b) we now argue by induction on $n$. If $n=1$ then $\Delta(\lambda)$ is one dimensional and hence irreducible, for all $\lambda$. Suppose now that $n>1$ and let $M \subset \Delta(\lambda)$ be a non-zero $\mathscr{W}_{r, n}(\mathbf{u})$-submodule of $\Delta(\lambda)$. By the remarks in the last paragraph, $M$ is spanned by a subset of $\left\{v_{\mathfrak{t}} \mid \mathfrak{t} \in \mathscr{T}_{n}^{u d}(\lambda)\right\}$. Therefore, if we consider $M$ as a $\mathscr{W}_{r, n-1}(\mathbf{u})$-module then $M \supset \Delta(\mu)$, for some multipartition $\mu$ which is obtained by adding or removing a node from $\lambda$.

Case 1. $|\lambda|=n$ : Since $|\lambda|=n$, The multipartition $\mu$ is obtained from $\lambda$ by removing a node. If $\lambda=\left((0), \ldots,(0),\left(a^{b}\right),(0), \ldots,(0)\right)$ then $\Delta(\lambda) \downarrow$ is irreducible as a $\mathscr{W}_{r, n-1}(\mathbf{u})$-module, so there is nothing to prove. Suppose then that $\lambda$ is not of this form and that $\nu$ is a different multipartition which is obtained from $\lambda$ by removing a node. Let $\mathfrak{t} \in \mathscr{T}_{n}^{u d}(\lambda)$ be an updown tableau such that $\mathfrak{t}_{n-1}=\mu$ and $\mu \backslash \mathfrak{t}_{n-2}=\lambda \backslash \nu$. So $v_{\mathfrak{t}} \in \Delta(\mu) \subset M$ and $\left(S_{n-1} \mathfrak{t}\right)_{n-1}=\nu$. Now,

$$
S_{n-1} v_{\mathfrak{t}}=a_{\mathfrak{t}}(n-1) v_{\mathfrak{t}}+b_{\mathfrak{t}}(n-1) v_{S_{n-1} \mathfrak{t}} \in M,
$$

and $b_{\mathfrak{t}}(n-1) \neq 0$ since $\lambda \backslash \mu$ and $\lambda \backslash \nu$ cannot be in the same row or in the same column. Consequently, $v_{S_{n-1} \mathfrak{t}} \in M$. This implies that $\Delta(\nu) \subset M$ since $\left(S_{n-1} \mathfrak{t}\right)_{n-1}=\nu$. Therefore, $\sum_{\nu \rightarrow \lambda} \Delta(\nu) \subset M$, so $M=\Delta(\lambda)$ by part (a). Hence, $\Delta(\lambda)$ is irreducible as required.

Case 2. $|\lambda|<n$ : Since $|\lambda|<n, \mathscr{T}_{n-2}^{u d}(\lambda)$ is non-empty so we fix $\mathfrak{u} \in$ $\mathscr{T}_{n-2}^{u d}(\lambda)$. Let $\mathfrak{t}=\left(\mathfrak{u}_{1}, \ldots, \mathfrak{u}_{n-2}, \mu, \lambda\right)$, then $\mathfrak{t} \in \mathscr{T}_{n}^{u d}(\lambda)$ and $v_{\mathfrak{t}} \in \Delta(\mu) \subset M$. Then

$$
E_{n-1} v_{\mathfrak{t}}=\sum_{\mathfrak{w}^{n-1} \mathfrak{t}} e_{\mathfrak{t w}}(n-1) v_{\mathfrak{w}} \in M
$$

As $e_{\mathfrak{t w}}(n-1) \neq 0$ whenever $\mathfrak{w} \stackrel{n-1}{\sim} \mathfrak{t}$, we have $v_{\mathfrak{w}} \in M$ for each term in this sum. If $\nu \leftarrow \lambda$ or $\nu \rightarrow \lambda$ then $\mathfrak{w}=\left(\mathfrak{u}_{1}, \ldots, \mathfrak{u}_{n-2}, \nu, \lambda\right) \stackrel{n-1}{\sim} \mathfrak{t}$, so $\Delta(\nu) \subset M$. Hence, $M=\Delta(\lambda)$ and $\Delta(\lambda)$ is irreducible as claimed. This completes the proof of (b).

Finally, we prove (c) and (d). We have already seen that the seminormal representations are pairwise non-isomorphic, so it remains to show that every irreducible is isomorphic to $\Delta(\lambda)$ for some $\lambda$. Let $\operatorname{Rad} \mathscr{W}_{r, n}(\mathbf{u})$ be the Jacobson radical of $\mathscr{W}_{r, n}(\mathbf{u})$. Then $\operatorname{dim}_{R} \mathscr{W}_{r, n}(\mathbf{u}) \geq \operatorname{dim}_{R}\left(\mathscr{W}_{r, n}(\mathbf{u}) / \operatorname{Rad} \mathscr{W}_{r, n}(\mathbf{u})\right) \geq \sum_{m=0}^{\lfloor n / 2\rfloor} \sum_{\lambda \vdash n-2 m}\left(\operatorname{dim}_{R} \Delta(\lambda)\right)^{2}$ 
By construction, $\operatorname{dim} \Delta(\lambda)=\# \mathscr{T}_{n}^{u d}(\lambda)=f^{(n, \lambda)}$. So using Corollary 5.2, and then Proposition 2.15, we have

$$
\operatorname{dim}_{R} \mathscr{W}_{r, n}(\mathbf{u}) \geq r^{n}(2 n-1) ! ! \geq \operatorname{dim}_{R} \mathscr{W}_{r, n}(\mathbf{u}) .
$$

Therefore, $\operatorname{Rad} \mathscr{W}_{r, n}(\mathbf{u})=0$, which forces $\operatorname{dim}_{R} \mathscr{W}_{r, n}(\mathbf{u})=r^{n}(2 n-1) !$ !. Now, parts (c) and (d) both follow from the Wedderburn-Artin Theorem.

Before establishing a strong version of Theorem $A$, we show that the Root conditions (Assumption 4.12) can be satisfied when $R=\mathbb{R}$.

LemmA 5.4. Suppose that $R=\mathbb{R}$ and we choose $u_{i} \in R$ in such a way that

a) $\left|u_{1}\right|>\cdots>\left|u_{r}\right| \geq n$ and $\left|u_{i}\right|-\left|u_{i+1}\right| \geq 2 n$,

b) $u_{i}<0$ if $i$ is even and $u_{i}>0$ if $i$ is odd.

Suppose that $\mathfrak{t} \in \mathscr{T}_{n}^{u d}(\lambda)$ and $1 \leq k<n$. Then $\left|a_{\mathfrak{t}}(k)\right| \leq 1$, if $\mathfrak{t}_{k-1} \neq \mathfrak{t}_{k+1}$, and $e_{\mathfrak{t t}}(k)>0$, if $\mathfrak{t}_{k-1}=\mathfrak{t}_{k+1}$. In particular, the Root Condition (4.12) holds if we choose non-negative square roots $\sqrt{b_{\mathfrak{t}}(k)} \geq 0$ and $\sqrt{e_{\mathfrak{t t}}(k)}>0$.

Proof. We start with the case $\mathfrak{t}_{k-1} \neq \mathfrak{t}_{k+1}$. Let $\alpha=\mathfrak{t}_{k} \ominus \mathfrak{t}_{k-1}$ and $\beta=$ $\mathfrak{t}_{k+1} \ominus \mathfrak{t}_{k}$. Note that $c(\alpha)+c(\beta) \neq 0$. Write $\alpha=(i, j, t)$ and $\beta=\left(i^{\prime}, j^{\prime}, t^{\prime}\right)$. If $t=t^{\prime}$ and both nodes are addable, or both nodes are removable, then $\alpha \neq \beta$. Thus, $c(\beta)-c(\alpha)$ is a nonzero integer and $\left|a_{\mathfrak{t}}(k)\right| \leq 1$. If $t=t^{\prime}$ and only one of the nodes is addable (and the other is removable), then

$$
\frac{1}{\left|a_{\mathfrak{u}}(k)\right|}=|c(\alpha)-c(\beta)|=\left|2 u_{t}+(j-i)+\left(j^{\prime}-i^{\prime}\right)\right| \geq 2\left|u_{t}\right|-2(n-1) \geq 2 .
$$

Hence, $\left|a_{t}(k)\right| \leq 1$ if $t=t^{\prime}$. A similar argument shows that $\left|a_{\mathfrak{t}}(k)\right| \leq 1$ when $t \neq t^{\prime}$.

Next we consider the case $\mathfrak{t}_{k-1}=\mathfrak{t}_{k+1}$. Let $\alpha=\mathfrak{t}_{k} \ominus \mathfrak{t}_{k-1}$ and $\lambda=\mathfrak{t}_{k-1}$. Write $\alpha=(i, j, t)$. By (4.8) and because $R=\mathbb{R}$, we have

$$
e_{\mathfrak{t t}}(k)=\left(2 c(\alpha)-(-1)^{r}\right) \prod_{\beta} \frac{c(\alpha)+c(\beta)}{c(\alpha)-c(\beta)},
$$

where $\beta$ runs over all of the addable and removable nodes of $\lambda$ with $\beta \neq \alpha$.

Suppose that $t$ is even. First we show that

$$
\prod_{\beta \notin \lambda(t)} \frac{c(\alpha)+c(\beta)}{c(\alpha)-c(\beta)}<0
$$


Consider the contents of all of the addable and removable nodes of $\lambda^{\left(t^{\prime}\right)}$, where $t^{\prime} \neq t$. If $t^{\prime}$ is even then there are $l$ positive contents $\left|u_{t^{\prime}}\right|+d_{j}$ with $\left|d_{j}\right|<n$, for $1 \leq j \leq l$, and $l+1$ negative contents $-\left|u_{t^{\prime}}\right|-c_{i}$ with $\left|c_{i}\right|<n$, for $1 \leq i \leq l+1$. Let $\varepsilon_{t^{\prime}}$ be the sign of the product of $\frac{c(\alpha)+c(\beta)}{c(\alpha)-c(\beta)}$ over all addable and removable nodes $\beta$ of $\lambda^{\left(t^{\prime}\right)}$. Our aim is to show that

$$
\prod_{t^{\prime} \neq t} \varepsilon_{t^{\prime}}=-1 \text {. }
$$

By our assumptions, $\varepsilon_{t^{\prime}}$ is equal to the sign of

$$
\frac{\left(-\left|u_{t}\right|+\left|u_{t^{\prime}}\right|\right)^{l}}{\left(-\left|u_{t}\right|-\left|u_{t^{\prime}}\right|\right)^{l}} \frac{\left(-\left|u_{t}\right|-\left|u_{t^{\prime}}\right|\right)^{l+1}}{\left(-\left|u_{t}\right|+\left|u_{t^{\prime}}\right|\right)^{l+1}}=\frac{\left|u_{t}\right|+\left|u_{t^{\prime}}\right|}{\left|u_{t}\right|-\left|u_{t^{\prime}}\right|} .
$$

Thus, $\varepsilon_{t^{\prime}}<0$ if and only if $t^{\prime}<t$. If $t^{\prime}$ is odd then there are $l+1$ positive contents $\left|u_{t^{\prime}}\right|+c_{i}$ with $\left|c_{i}\right|<n$, for $1 \leq i \leq l+1$, and $l$ negative contents $-\left|u_{t^{\prime}}\right|-d_{j}$ with $\left|d_{j}\right|<n$, for $1 \leq j \leq l$. Then, by the same argument, $\varepsilon_{t^{\prime}}<0$ if and only if $t^{\prime}<t$ again. Thus

$$
\prod_{t^{\prime} \neq t} \varepsilon_{t^{\prime}}=(-1)^{t-1}=-1
$$

Let $-\left|u_{t}\right|-c_{i}$, for $1 \leq i \leq l+1$, be the contents of the addable nodes of $\lambda^{(t)}$ and let $\left|u_{t}\right|+d_{j}$, for $1 \leq j \leq l$, be the contents of the removable nodes of $\lambda^{(t)}$. We may assume that

$$
c_{1}>d_{1}>\cdots>c_{l}>d_{l}>c_{l+1} .
$$

Let $\varepsilon_{t}$ be the sign of the product of $\frac{c(\alpha)+c(\beta)}{c(\alpha)-c(\beta)}$, where $\beta$ runs over all of the addable and removable nodes of $\lambda^{(t)}$ such that $\beta \neq \alpha$.

If $c(\alpha)=-\left|u_{t}\right|-c_{i}$, for some $i$, then $\varepsilon_{t}$ is equal to the sign of

$$
\prod_{k \neq i} \frac{-2\left|u_{t}\right|-c_{i}-c_{k}}{c_{k}-c_{i}} \prod_{k=1}^{l} \frac{d_{k}-c_{i}}{-2\left|u_{t}\right|-c_{i}-d_{k}}
$$

so $\varepsilon_{t}=\frac{(-1)^{l}}{(-1)^{l+1-i}} \frac{(-1)^{l-i+1}}{(-1)^{l}}=1$. As $2 c(\alpha)-(-1)^{r}=-2\left|u_{t}\right|-2 c_{i} \pm 1<0$ and

$$
\prod_{1 \leq t^{\prime} \leq r} \varepsilon_{t^{\prime}}=-1
$$

we have $e_{\mathfrak{t t}}(k)>0$. 
If $c(\alpha)=\left|u_{t}\right|+d_{j}$, for some $j$, then $\varepsilon_{t}$ is equal to the sign of

$$
\prod_{k=1}^{l+1} \frac{d_{j}-c_{k}}{2\left|u_{t}\right|+d_{j}+c_{k}} \prod_{k \neq j}^{l} \frac{2\left|u_{t}\right|+d_{j}+d_{k}}{d_{j}-d_{k}},
$$

so $\varepsilon_{t}=\frac{(-1)^{j}}{(-1)^{j-1}}=-1$. As $2 c(\alpha)-(-1)^{r}=2\left|u_{t}\right|+2 d_{j} \pm 1>0$ and

$$
\prod_{1 \leq t^{\prime} \leq r} \varepsilon_{t^{\prime}}=-1
$$

we have $e_{\mathfrak{t t}}(k)>0$ again.

The case when $t$ is odd is handled similarly. In this case, we have

$$
\prod_{\beta \notin \lambda^{(t)}} \frac{c(\alpha)+c(\beta)}{c(\alpha)-c(\beta)}>0,
$$

because its sign is equal to $(-1)^{t-1}=1$. Let $\left|u_{t}\right|+c_{i}$, for $1 \leq i \leq l+1$, be the contents of the addable nodes of $\lambda^{(t)}$ and let $-\left|u_{t}\right|-d_{j}$, for $1 \leq j \leq l$, be the contents of the removable nodes of $\lambda^{(t)}$ such that

$$
c_{1}>d_{1}>\cdots>c_{l}>d_{l}>c_{l+1}
$$

If $c(\alpha)=\left|u_{t}\right|+c_{i}$, for some $i$, then $\varepsilon_{t}$ is equal to the sign of

$$
\prod_{k \neq i} \frac{2\left|u_{t}\right|+c_{i}+c_{k}}{c_{i}-c_{k}} \prod_{k=1}^{l} \frac{c_{i}-d_{k}}{2\left|u_{t}\right|+c_{i}+d_{k}}
$$

so $\varepsilon_{t}=\frac{(-1)^{i-1}}{(-1)^{i-1}}=1$. As $2 c(\alpha)-(-1)^{r}>0$ we have $e_{\mathfrak{t t}}(k)>0$.

If $c(\alpha)=-\left|u_{t}\right|-d_{j}$, for some $j$, then $\varepsilon_{t}$ is equal to the sign of

$$
\prod_{k=1}^{l+1} \frac{c_{k}-d_{j}}{-2\left|u_{t}\right|-d_{j}-c_{k}} \prod_{k \neq j}^{l} \frac{-2\left|u_{t}\right|-d_{j}-d_{k}}{d_{k}-d_{j}}
$$

so $\varepsilon_{t}=\frac{(-1)^{l-j+1}}{(-1)^{l+1}} \frac{(-1)^{l-1}}{(-1)^{l-j}}=-1$. As $2 c(\alpha)-(-1)^{r}<0$ we have $e_{\mathfrak{t t}}(k)>0$ again.

We can now prove a stronger version of Theorem A. 
THEOREM 5.5. Suppose that $R$ is a commutative ring in which 2 is invertible and that $\Omega$ is $\mathbf{u}$-admissible. Then $\mathscr{W}_{r, n}(\mathbf{u})$ is free as an $R$-module with basis the set of r-regular monomials. Consequently, $\mathscr{W}_{r, n}(\mathbf{u})$ is free of rank $r^{n}(2 n-1) ! !$.

Proof. Recall that if $R$ is a ring in which 2 is invertible then $\mathscr{W}_{r, n}(\mathbf{u})$ is spanned by the set of $r$-regular monomials by Proposition 2.15. For convenience, if $S$ is a ring and $\mathbf{u}_{s} \in S^{r}$ then we let $\mathscr{W}_{S}\left(\mathbf{u}_{S}\right)$ be the cyclotomic Nazarov-Wenzl algebra defined over $S$ with parameters $\mathbf{u}_{S}$.

First, we consider the special case when $R=\mathcal{Z}$, where $\mathcal{Z}=$ $\mathbb{Z}\left[\frac{1}{2}, \dot{u}_{1}, \ldots, \dot{u}_{r}\right]$ and the $\dot{u}_{i}$ are indeterminates over $\mathbb{Z}$. Let $\dot{\mathbf{u}}=\left(\dot{u}_{1}, \ldots, \dot{u}_{r}\right)$, define $\dot{\Omega}$ in accordance with Definition 3.6 and consider the cyclotomic Nazarov-Wenzl algebra $\mathscr{W}_{\mathcal{Z}}(\dot{\mathbf{u}})$. As $\mathbb{R}$ is not finitely generated over $\mathbb{Q}$ we can find $r$ algebraically independent transcendental real numbers $u_{1}^{\prime}, \ldots, u_{r}^{\prime} \in \mathbb{R}$ which satisfy the hypotheses of Lemma 5.4. Let $\mathcal{Z}^{\prime}=\mathbb{Z}\left[\frac{1}{2}, u_{1}^{\prime}, \ldots, u_{r}^{\prime}\right]$ and let $\theta: \mathcal{Z} \rightarrow \mathcal{Z}^{\prime}$ be the $\mathcal{Z}$-linear map determined by $\theta\left(\dot{u}_{i}\right)=u_{i}^{\prime}$, for $1 \leq i \leq r$. Then $\theta$ is a ring isomorphism. Let $\mathbf{u}^{\prime}=\left(u_{1}^{\prime}, \ldots, u_{r}^{\prime}\right)$ and $\Omega^{\prime}=\left\{\theta\left(\dot{\omega}_{a}\right) \mid a \geq 0\right\}$. Then $\Omega^{\prime}$ is $\mathbf{u}^{\prime}$-admissible and $\theta$ induces an isomorphism of $\mathcal{Z}$-algebras $\mathscr{W}_{\mathcal{Z}}(\dot{\mathbf{u}}) \cong \mathscr{W}_{\mathcal{Z}^{\prime}}\left(\mathbf{u}^{\prime}\right)$, where the inverse map is the homomorphism induced by $\theta^{-1}: \mathcal{Z}^{\prime} \rightarrow \mathcal{Z}$.

Now, by Lemma 5.4 and Theorem $5.3(\mathrm{~d}), \mathscr{W}_{\mathbb{R}}\left(\mathbf{u}^{\prime}\right)$ is an $\mathbb{R}$-algebra of dimension $r^{n}(2 n-1)$ !!. Hence the set of $r$-regular monomials is an $\mathbb{R}$-basis of $\mathscr{W}_{\mathbb{R}}\left(\mathbf{u}^{\prime}\right)$ since there are $r^{n}(2 n-1) ! ! r$-regular monomials. In particular, the set of $r$-regular monomials is linearly independent over $\mathbb{R}$, and hence linearly independent over $\mathcal{Z}^{\prime}$. Therefore, $\mathscr{W}_{\mathcal{Z}^{\prime}}\left(\mathbf{u}^{\prime}\right)$ is free as a $\mathcal{Z}^{\prime}$-module of rank $r^{n}(2 n-1)$ !!. Hence, $\mathscr{W}_{\mathcal{Z}}(\dot{\mathbf{u}})$ is free as a $\mathcal{Z}$-module of rank $r^{n}(2 n-1)$ !!.

Now suppose that $R$ is an arbitrary commutative ring (in which 2 is invertible). Then we can consider $R$ as a $\mathcal{Z}$-algebra by letting $\dot{u}_{i}$ act on $R$ as multiplication by $u_{i}$, for $1 \leq i \leq r$. Since $\mathscr{W}_{\mathcal{Z}}(\dot{\mathbf{u}})$ is $\mathcal{Z}$-free, the $R$ algebra $\mathscr{W}_{\mathcal{Z}}(\dot{\mathbf{u}}) \otimes_{\mathcal{Z}} R$ is free as an $R$-module of rank $r^{n}(2 n-1)$ !!. As the generators of $\mathscr{W}_{\mathcal{Z}}(\dot{\mathbf{u}}) \otimes_{\mathcal{Z}} R$ satisfy the relations of $\mathscr{W}_{r, n}(\mathbf{u})=\mathscr{W}_{R}(\mathbf{u})$ we have a surjective homomorphism $\mathscr{W}_{r, n}(\mathbf{u}) \rightarrow \mathscr{W}_{\mathcal{Z}}(\dot{\mathbf{u}}) \otimes_{\mathcal{Z}} R$. By Proposition 2.15 this map must be an isomorphism, so we are done.

As an easy application of the Theorem we obtain the following useful fact which we will use many times below without mention.

Proposition 5.6. Suppose that $R$ is a commutative ring in which 2 is invertible and that $\Omega$ is $\mathbf{u}$-admissible. 
a) For $1 \leq m \leq n$, let $\mathscr{W}_{r, m}^{\prime}(\mathbf{u})$ be the subalgebra of $\mathscr{W}_{r, n}(\mathbf{u})$ generated by $\left\{S_{i}, E_{i}, X_{j} \mid 1 \leq i<m\right.$ and $\left.1 \leq j \leq m\right\}$. Then $\mathscr{W}_{r, m}^{\prime}(\mathbf{u}) \cong \mathscr{W}_{r, m}(\mathbf{u})$.

b) The Brauer algebra $\mathscr{B}_{n}\left(\omega_{0}\right)$ is isomorphic to the subalgebra of $\mathscr{W}_{r, n}(\mathbf{u})$ generated by $\left\{S_{i}, E_{i} \mid 1 \leq i<n\right\}$.

\section{$\S 6$. The degenerate Hecke algebras of type $G(r, 1, n)$}

Suppose $R$ is a commutative ring and let $\mathbf{u} \in R^{r}$. Recall from Section 2 that $\mathscr{H}_{r, n}(\mathbf{u})$ is the degenerate Hecke algebra with parameters $\mathbf{u}$. In this section we give several results from the representation theory of $\mathscr{H}_{r, n}(\mathbf{u})$ which we will need in our study of the cyclotomic Nazarov-Wenzl algebras. As the proofs of these results are very similar to (and easier than) the proofs of the corresponding results for the Ariki-Koike algebras we are very brief with the details.

The following result is proved by Kleshchev [Kle05]. We use the seminormal representations of $\mathscr{W}_{r, n}(\mathbf{u})$ to give another proof.

Let $\Lambda_{r}^{+}(n)$ be the set of $r$-multipartitions of $n$. We consider $\Lambda_{r}^{+}(n)$ as a partially ordered set under dominance $\unrhd$, where $\lambda \unrhd \mu$ if

$$
\sum_{t=1}^{s-1}\left|\lambda^{(t)}\right|+\sum_{j=1}^{k} \lambda_{k}^{(s)} \geq \sum_{t=1}^{s-1}\left|\mu^{(t)}\right|+\sum_{j=1}^{k} \mu_{k}^{(s)},
$$

for $1 \leq s \leq r$ and all $k \geq 0$. If $\lambda \unrhd \mu$ and $\lambda \neq \mu$ we sometimes write $\lambda \triangleright \mu$.

THEOREM 6.1. The degenerate Hecke algebra $\mathscr{H}_{r, n}(\mathbf{u})$ is free as an $R$ module of rank $r^{n} n$ !.

Proof. It is not difficult to see that for any $\operatorname{ring} R$ set

$$
\left\{Y_{1}^{k_{1}} Y_{2}^{k_{2}} \cdots Y_{n}^{k_{n}} T_{w} \mid 0 \leq k_{i} \leq r-1, w \in \mathfrak{S}_{n}\right\}
$$

spans $\mathscr{H}_{r, n}(\mathbf{u})$ as an $R$-module. So we need to prove that these elements are linearly independent.

We adopt the notation from the proof of Theorem 5.5. As in the proof of that result, we first consider the case when $R=\mathcal{Z}$, where $\mathcal{Z}=$ $\mathbb{Z}\left[\frac{1}{2}, \dot{u}_{1}, \ldots, \dot{u}_{r}\right]$, and we choose $r$ algebraically independent transcendental real numbers $u_{1}^{\prime}, \ldots, u_{r}^{\prime}$ which satisfy the hypotheses of Lemma 5.4. Let $\mathcal{Z}^{\prime}=\mathbb{Z}\left[\frac{1}{2}, u_{1}^{\prime}, \ldots, u_{r}^{\prime}\right]$. Then $\mathcal{Z} \cong \mathcal{Z}^{\prime} \hookrightarrow \mathbb{R}$ and we can ask whether the degenerate Hecke algebra $\mathscr{H}_{\mathbb{R}}\left(\mathbf{u}^{\prime}\right)$, defined over $\mathbb{R}$ and with parameters $\mathbf{u}^{\prime}=\left(u_{1}^{\prime}, \ldots, u_{r}^{\prime}\right)$, acts on the seminormal representations of $\mathscr{W}_{\mathbb{R}}\left(\mathbf{u}^{\prime}\right)$. By 
definition, if $\lambda \in \Lambda_{r}^{+}(n)$ then $E_{i} \Delta(\lambda)=0$, for $1 \leq i<n$. Therefore, over $\mathbb{R}$, $\Delta(\lambda)$ can be considered as an $\mathscr{H}_{\mathbb{R}}\left(\mathbf{u}^{\prime}\right)$-module by Corollary 2.16. Hence, as in the proof of Theorem 5.5,

$$
\operatorname{dim}_{\mathbb{R}} \mathscr{H}_{\mathbb{R}}\left(\mathbf{u}^{\prime}\right) \geq \sum_{\lambda \in \Lambda_{r}^{+}(n)}\left(\operatorname{dim}_{\mathbb{R}} \Delta(\lambda)\right)^{2}=r^{n} n !
$$

Consequently, by the opening paragraph of the proof, this set is a basis of $\mathscr{H}_{\mathbb{R}}\left(\mathbf{u}^{\prime}\right)$. As in the proof of Theorem 5.5 it follows that $\mathscr{H}_{\mathcal{Z}}(\dot{\mathbf{u}})$ is free as a $\mathcal{Z}$-module of rank $r^{n} n$ !. The result for a general ring $R$ now follows by a specialization argument.

We remark that the definition of the seminormal representations of $\mathscr{W}_{r, n}(\mathbf{u})$ required that $R$ satisfy assumption (4.12). It is not hard to modify the definition of the seminormal representations of $\mathscr{H}_{r, n}(\mathbf{u})$ so that the formulae do not involve any square roots and so that they work over an arbitrary field (cf. [AK94]). In particular, this leads to a simplification of the last argument.

Definition 6.2. (Graham and Lehrer [GL96]) Let $R$ be a commutative ring and $A$ an $R$-algebra. Fix a partially ordered set $\Lambda=(\Lambda, \unrhd)$ and for each $\lambda \in \Lambda$ let $T(\lambda)$ be a finite set. Finally, fix $C_{\mathfrak{s t}}^{\lambda} \in A$ for all $\lambda \in \Lambda$ and $\mathfrak{s}, \mathfrak{t} \in T(\lambda)$.

Then the triple $(\Lambda, T, C)$ is a cell datum for $A$ if:

a) $\left\{C_{\mathfrak{s t}}^{\lambda} \mid \lambda \in \Lambda\right.$ and $\left.\mathfrak{s}, \mathfrak{t} \in T(\lambda)\right\}$ is an $R$-basis for $A$;

b) the $R$-linear map $*: A \rightarrow A$ determined by $\left(C_{\mathfrak{s t}}^{\lambda}\right)^{*}=C_{\mathfrak{t} \mathfrak{s}}^{\lambda}$, for all $\lambda \in \Lambda$ and all $\mathfrak{s}, \mathfrak{t} \in T(\lambda)$ is an anti-isomorphism of $A$;

$c$ ) for all $\lambda \in \Lambda, \mathfrak{s} \in T(\lambda)$ and $a \in A$ there exist scalars $r_{\mathfrak{s u}}(a) \in R$ such that

$$
a C_{\mathfrak{s t}}^{\lambda}=\sum_{\mathfrak{u} \in T(\lambda)} r_{\mathfrak{s u}}(a) C_{\mathfrak{u t}}^{\lambda} \quad\left(\bmod A^{\triangleright \lambda}\right),
$$

where $A^{\triangleright \lambda}=R$-span $\left\{C_{\mathfrak{u v}}^{\mu} \mid \mu \triangleright \lambda\right.$ and $\left.\mathfrak{u}, \mathfrak{v} \in T(\mu)\right\}$.

An algebra $A$ is a cellular algebra if it has a cell datum and in this case we call $\left\{C_{\mathfrak{s t}}^{\lambda} \mid \mathfrak{s}, \mathfrak{t} \in T(\lambda), \lambda \in \Lambda\right\}$ a cellular basis of $A$.

To show that $\mathscr{H}_{r, n}(\mathbf{u})$ is a cellular algebra we modify the construction of the Murphy basis of the Ariki-Koike algebras; see [DJM99]. For any multipartition $\lambda=\left(\lambda^{(1)}, \lambda^{(2)}, \ldots, \lambda^{(r)}\right)$ we define $u_{\lambda}=u_{a_{1}, 1} u_{a_{2}, 2} \cdots u_{a_{r-1}, r-1}$, 
where $u_{a, i}=\left(Y_{1}-u_{i+1}\right)\left(Y_{2}-u_{i+1}\right) \cdots\left(Y_{a}-u_{i+1}\right)$ and $a_{i}=\sum_{j=1}^{i}\left|\lambda^{(j)}\right|$, $1 \leq i \leq r-1$. Let $\mathfrak{S}_{\lambda}$ be the Young subgroup $\mathfrak{S}_{\lambda^{(1)}} \times \mathfrak{S}_{\lambda^{(2)}} \times \cdots \times \mathfrak{S}_{\lambda^{(r)}}$ of $\mathfrak{S}_{n}$. Let $x_{\lambda}=\sum_{w \in \mathfrak{S}_{\lambda}} T_{w}$ and define

$$
m_{\mathfrak{s t}}=T_{d(\mathfrak{s})^{-1}} u_{\lambda} x_{\lambda} T_{d(\mathfrak{t})} \in \mathscr{H}_{r, n}(\mathbf{u}),
$$

where $\mathfrak{s}, \mathfrak{t}$ are standard $\lambda$-tableaux.

TheOREM 6.3. The set $\left\{m_{\mathfrak{s t}} \mid \mathfrak{s}, \mathfrak{t} \in \mathscr{T}^{\text {std }}(\lambda)\right.$ and $\left.\lambda \in \Lambda_{r}^{+}(n)\right\}$ is a cellular basis of $\mathscr{H}_{r, n}(\mathbf{u})$.

Proof. The proof of this result is similar to, but much easier than, the corresponding result for the cyclotomic Hecke algebras. See [DJM99] for details.

We next give a formula for the Gram determinant of the cell modules of $\mathscr{H}_{r, n}(\mathbf{u})$. This requires some definitions.

Definition 6.4. The parameters $\mathbf{u}=\left(u_{1}, \ldots, u_{r}\right)$ are generic for $\mathscr{H}_{r, n}(\mathbf{u})$ if whenever there exists $d \in \mathbb{Z}$ such that $u_{i}-u_{j}=d \cdot 1_{R}$ then $|d| \geq n$.

The following Lemma is well-known (cf. [JM00, Lemma 3.12]), and is easily verified by induction on $n$.

LEMMA 6.5. Suppose that the parameters $\mathbf{u}$ are generic for $\mathscr{H}_{r, n}(\mathbf{u})$ and that $R$ is a field with char $R>n$. Let $\lambda$ and $\mu$ be multipartitions of $n$ and suppose that $\mathfrak{s} \in \mathscr{T}_{n}^{\text {std }}(\lambda)$ and $\mathfrak{t} \in \mathscr{T}_{n}^{\text {std }}(\mu)$. Then $\mathfrak{s}=\mathfrak{t}$ if and only if $c_{\mathfrak{s}}(k)=c_{\mathfrak{t}}(k)$, for $k=1, \ldots, n$.

As in the definition of a cellular basis, if $\lambda \in \Lambda_{r}^{+}(n)$ then we let $\mathscr{H}_{r, n}^{\triangleright \lambda}$ be the free $R$-submodule $\mathscr{H}_{r, n}(\mathbf{u})$ with basis $\left\{m_{\mathfrak{s t}} \mid \mathfrak{s}, \mathfrak{t} \in \mathscr{T}^{\text {std }}(\mu)\right.$ for $\left.\mu \triangleright \lambda\right\}$. It follows directly from Definition 6.2(c) that $\mathscr{H}_{r, n}^{\triangleright \lambda}$ is a two-sided ideal of $\mathscr{H}_{r, n}$.

Lemma 6.6. Suppose that $\lambda$ is a multipartition of $n$ and that $\mathfrak{s , t} \in$ $\mathscr{T}_{n}^{s t d}(\lambda)$. Then

$$
Y_{k} m_{\mathfrak{s t}}=c_{\mathfrak{s}}(k) m_{\mathfrak{s t}}+\sum_{\substack{\mathfrak{u} \in \mathscr{T}_{n}^{s t d}(\lambda) \\ \mathfrak{u} \triangleright \mathfrak{s}}} r_{\mathfrak{u t}} m_{\mathfrak{u t}} \quad\left(\bmod \mathscr{H}_{r, n}^{\triangleright \lambda}\right)
$$

for some $r_{\mathfrak{u t}} \in R$. 
Proof. If $r=1$ then this is a result of Murphy's [Mur83]. The general case can be deduced from this following the argument of [JM00, Prop. 3.7].

We can now follow the arguments of [Mat04] to construct a "seminormal" basis of $\mathscr{H}_{r, n}(\mathbf{u})$.

Definition 6.7. Suppose that $\lambda \in \Lambda_{r}^{+}(n)$.

a) For each $\mathfrak{t} \in \mathscr{T}^{s t d}(\lambda)$ let

$$
F_{\mathfrak{t}}=\prod_{k=1}^{n} \prod_{\substack{\mu \in \Lambda_{r}^{+}(n) \\ \mathfrak{u} \in \mathscr{T}^{s t d}(\mu) \\ c_{\mathfrak{u}}(k) \neq c_{\mathfrak{t}}(k)}} \frac{Y_{k}-c_{\mathfrak{u}}(k)}{c_{\mathfrak{t}}(k)-c_{\mathfrak{u}}(k)} .
$$

b) If $\mathfrak{s}, \mathfrak{t} \in \mathscr{T}^{\text {std }}(\lambda)$ then let $f_{\mathfrak{s t}}=F_{\mathfrak{s}} m_{\mathfrak{s t}} F_{\mathfrak{t}}$.

Using the last two results and the definitions it is not hard to show that if $\mathfrak{s}, \mathfrak{t}$ and $\mathfrak{u}$ are standard tableaux then $f_{\mathfrak{s t}} F_{\mathfrak{u}}=\delta_{\mathfrak{t u}} f_{\mathfrak{s t}}$; see, for example, [Mat99, Prop. 3.35]. Hence, from Theorem 6.3 and Lemma 6.6 we obtain the following.

Proposition 6.8. Suppose that $R$ is a field with char $R>n$ and that $\mathbf{u}$ is generic for $\mathscr{H}_{r, n}(\mathbf{u})$. Then $\left\{f_{\mathfrak{s t}} \mid \mathfrak{s}, \mathfrak{t} \in \mathscr{T}^{\text {std }}(\lambda), \lambda \in \Lambda_{r}^{+}(n)\right\}$ is a basis of $\mathscr{H}_{r, n}(\mathbf{u})$. Moreover, for each standard tableau $\mathfrak{t}$ there exists a scalar $\gamma_{\mathfrak{t}} \in R$ such that

$$
f_{\mathfrak{s t}} f_{\mathfrak{u v}}=\delta_{\mathfrak{t u}} \gamma_{\mathfrak{t}} f_{\mathfrak{s v}}
$$

where $\mathfrak{s}, \mathfrak{t} \in \mathscr{T}^{\text {std }}(\lambda), \mathfrak{u}, \mathfrak{v} \in \mathscr{T}^{\text {std }}(\mu)$, and $\lambda, \mu \in \Lambda_{r}^{+}(n)$.

Notice, in particular, that the Proposition implies that $\left\{f_{\mathfrak{s t}}\right\}$ is also a cellular basis of $\mathscr{H}_{r, n}(\mathbf{u})$.

Although we will not pursue this here, we remark that $F_{\mathfrak{t}}=\frac{1}{\gamma_{\mathfrak{t}}} f_{\mathfrak{t t}}$ and that these elements give a complete set of pairwise orthogonal primitive idempotents for $\mathscr{H}_{r, n}(\mathbf{u})$. This can be proved by repeating the argument of [Mat04, Theorem 2.15]

Suppose that $\lambda$ is a multipartition of $n$ and let $S(\lambda)$ be the associated Specht module, or cell module, of $\mathscr{H}_{r, n}(\mathbf{u})$. Thus, $S(\lambda)$ is the free $R$-module 
with basis $\left\{m_{\mathfrak{s}} \mid \mathfrak{s} \in \mathscr{T}^{s t d}(\lambda)\right\}$ and where the action of $\mathscr{H}_{r, n}(\mathbf{u})$ on $S(\lambda)$ is given by

$$
a m_{\mathfrak{s}}=\sum_{\mathfrak{u} \in \mathscr{T}^{s t d}(\lambda)} r_{\mathfrak{s u}}(a) m_{\mathfrak{u}},
$$

where the scalars $r_{\mathfrak{s u}}(a) \in R$ are as in Definition 6.2(c).

It follows directly from Definition 6.2 that $S(\lambda)$ comes equipped with a symmetric bilinear form $\langle$,$\rangle which is determined by$

$$
\left\langle m_{\mathfrak{s}}, m_{\mathfrak{t}}\right\rangle m_{\mathfrak{u v}} \equiv m_{\mathfrak{u s}} m_{\mathfrak{t v}} \quad\left(\bmod \mathscr{H}_{r, n}^{\triangleright \lambda}\right)
$$

for $\mathfrak{s}, \mathfrak{t}, \mathfrak{u}, \mathfrak{v} \in \mathscr{T}^{s t d}(\lambda)$. Let $G(\lambda)=\operatorname{det}\left(\left\langle m_{\mathfrak{s}}, m_{\mathfrak{t}}\right\rangle\right)$, for $\mathfrak{s}, \mathfrak{t} \in \mathscr{T}^{s t d}(\lambda)$, be the Gram determinant of this form. So $\mathcal{G}(\lambda)$ is well-defined up to a unit in $R$.

Corollary 6.9. Suppose that $R$ is a field with char $R>n$ and that $\mathbf{u}$ is generic for $\mathscr{H}_{r, n}=\mathscr{H}_{r, n}(\mathbf{u})$. Let $\lambda$ be a multipartition of $n$. Then

$$
\mathcal{G}(\lambda)=\prod_{\mathfrak{t} \in \mathscr{T}^{s t d}(\lambda)} \gamma_{\mathfrak{t}}
$$

Proof. Fix $\mathfrak{t} \in \mathscr{T}^{s t d}(\lambda)$. Then Specht module $S(\lambda)$ is isomorphic to the submodule of $\mathscr{H}_{r, n} / \mathscr{H}_{r, n}^{\triangleright \lambda}$ which is spanned by $\left\{m_{\mathfrak{s t}}+\mathscr{H}_{r, n}^{\triangleright \lambda} \mid \mathfrak{s} \in \mathscr{T}^{s t d}(\lambda)\right\}$, where the isomorphism is given by $\theta: \mathscr{H}_{r, n} / \mathscr{H}_{r, n}^{\triangleright \lambda} \rightarrow S(\lambda) ; m_{\mathfrak{s t}}+\mathscr{H}_{r, n}^{\triangleright \lambda} \mapsto$ $m_{\mathfrak{s}}$. Let $f_{\mathfrak{s}}=\theta\left(f_{\mathfrak{s t}}\right)$. Then $\left\{f_{\mathfrak{s}} \mid \mathfrak{s} \in \mathscr{T}^{\text {std }}(\lambda)\right\}$ is a basis of $S(\lambda)$ and the transition matrix between the two bases $\left\{m_{\mathfrak{s}}\right\}$ and $\left\{f_{\mathfrak{s}}\right\}$ of $S(\lambda)$ is unitriangular by Lemma 6.6. Consequently, $\mathcal{G}(\lambda)=\operatorname{det}\left(\left\langle f_{\mathfrak{s}}, f_{\mathfrak{t}}\right\rangle\right)$, where $\mathfrak{s}, \mathfrak{t} \in \mathscr{T}^{\text {std }}(\lambda)$. However, it follows from the multiplication formulae in Proposition 6.8 that $\left\langle f_{\mathfrak{s}}, f_{\mathfrak{t}}\right\rangle=\delta_{\mathfrak{s t}} \gamma_{\mathfrak{t}}$; see the proof of [Mat04, Theorem 2.11] for details. Hence the result.

Consequently, in order to compute $\mathcal{G}(\lambda)$ it is sufficient to determine $\gamma_{t}$, for all $\mathfrak{t} \in \mathscr{T}^{s t d}(\lambda)$. It is possible to give an explicit closed formula for $\gamma_{\mathfrak{t}}$ (cf. [Mat04, (2.8)]), however, the following recurrence relation is easier to check and sufficient for our purposes.

Given two standard $\lambda$-tableaux $\mathfrak{s}$ and $\mathfrak{t}$ write $\mathfrak{s} \unrhd \mathfrak{t}$ if $\mathfrak{s}_{k} \unrhd \mathfrak{t}_{k}$, for $1 \leq k \leq n$. Let $\mathfrak{t}^{\lambda}$ be the unique standard $\lambda$-tableaux such that $\mathfrak{t}^{\lambda} \unrhd \mathfrak{s}$ for all $\mathfrak{s} \in \mathscr{T}^{\text {std }}(\lambda)$. If $\mathfrak{s} \unrhd \mathfrak{t}$ and $\mathfrak{s} \neq \mathfrak{t}$ then we write $\mathfrak{s} \triangleright \mathfrak{t}$.

Lemma 6.10. Suppose that $R$ is generic for $\mathscr{H}_{r, n}(\mathbf{u})$ and that char $R>$ $n$. Let $\lambda$ be a multipartition of $n$. 
a) $\gamma_{\mathfrak{t}^{\lambda}}=\prod_{1 \leq t \leq r} \prod_{i \geq 1}\left(\lambda_{i}^{(t)}\right) ! \cdot \prod_{1 \leq s<t \leq r} \prod_{\substack{i, j \geq 1 \\ 1 \leq j \leq \lambda_{i}^{(s)}}}\left(j-i+u_{s}-u_{t}\right)$.

b) Suppose that $\mathfrak{s}, \mathfrak{t} \in \mathscr{T}^{\text {std }}(\lambda)$ such that $\mathfrak{s} \triangleright \mathfrak{t}$ and $\mathfrak{s}=S_{k} \mathfrak{t}$, for some $k$. Then $\gamma_{\mathfrak{t}}=\frac{\left(c_{\mathfrak{s}}(k)-c_{\mathfrak{t}}(k)+1\right)\left(c_{\mathfrak{s}}(k)-c_{\mathfrak{t}}(k)-1\right)}{\left(c_{\mathfrak{s}}(k)-c_{\mathfrak{t}}(k)\right)^{2}} \gamma_{\mathfrak{s}}$.

Proof. Part (a) follows easily by induction on $n$. Part (b) follows using arguments similar to [JM00, Cor. 3.14 and Prop. 3.19].

We remark that the arguments of [JM00, 3.30-3.37] can now be adapted to give a closed formula for $\mathcal{G}(\lambda)$. The final result is that

$$
\mathcal{G}(\lambda)=\prod_{\nu \in \Lambda_{r}^{+}(n)} g_{\lambda \nu}^{\left|\mathscr{T}^{s t d}(\lambda)\right|}
$$

where $g_{\lambda \nu}$ is a product of terms of the form $\left(c_{\mathfrak{t}^{\lambda}}(k)-c_{\mathfrak{t}^{\nu}}(l)\right)^{ \pm 1}$, where these terms are determined in exactly the same way as in [JM00, Defn 3.36]. As we do not need the precise formula we leave the details to the interested reader.

TheOREm 6.11. Suppose that $R$ is a field and that $\mathbf{u} \in R^{r}$. Then $\mathscr{H}_{r, n}(\mathbf{u})$ is (split) semisimple if and only if char $R>n$ and $\mathbf{u}$ is generic for $\mathscr{H}_{r, n}(\mathbf{u})$.

Proof. First, note that because $\mathscr{H}_{r, n}(\mathbf{u})$ is cellular, it is semisimple if and only if it is split semisimple; see, for example, [Mat99, Cor. 2.21].

Next, suppose that char $R>n$ and that $\mathbf{u}$ is generic for $\mathscr{H}_{r, n}(\mathbf{u})$. Then $\mathcal{G}(\lambda) \neq 0$ for all $\lambda \in \Lambda_{r}^{+}(n)$ by Lemma 6.10. Consequently, for each $\lambda \in$ $\Lambda_{r}^{+}(n)$ the Specht module $S(\lambda)$ is irreducible. Hence, by [Mat99, Cor 2.21] again, $\mathscr{H}_{r, n}(\mathbf{u})$ is semisimple.

To prove the converse, let $\lambda=((n),(0), \ldots,(0)) \in \Lambda_{r}^{+}(n)$ and set $m_{\lambda}=$ $m_{\mathfrak{t}^{\lambda} \mathfrak{t}^{\lambda}} ;$ more explicitly,

$$
m_{\lambda}=\sum_{w \in \mathfrak{S}_{n}} T_{w} \cdot \prod_{t=2}^{r} \prod_{k=1}^{n}\left(Y_{k}-u_{t}\right) .
$$

It is easy to see that $T_{\sigma} m_{\lambda}=m_{\lambda}=m_{\lambda} T_{\sigma}$, for any $\sigma \in \mathfrak{S}_{n}$. It also follows from Lemma 6.6 that $Y_{k} m_{\lambda}=c_{\mathfrak{t}^{\lambda}}(k) m_{\lambda}=m_{\lambda} Y_{k}$. Hence, $\mathscr{H}_{r, n}(\mathbf{u}) m_{\lambda}$ 
$\mathscr{H}_{r, n}(\mathbf{u})=R m_{\lambda}$ and

$$
m_{\lambda}^{2}=n ! \prod_{t=2}^{r} \prod_{d=0}^{n-1}\left(u_{1}+d-u_{t}\right) \cdot m_{\lambda}
$$

If char $R \leq n$ then $n !=0$ in $R$ so that $R m_{\lambda}$ is a nilpotent ideal in $\mathscr{H}_{r, n}(\mathbf{u})$, so $\mathscr{H}_{r, n}(\mathbf{u})$ is not semisimple. On the other hand if $\mathbf{u}$ is not generic for $\mathscr{H}_{r, n}(\mathbf{u})$ then $u_{i}-u_{j}=d 1_{R}$ for some $i \neq j$ and some $d \in \mathbb{Z}$ with $|d|<n$. By renumbering $u_{1}, \ldots, u_{r}$, if necessary, we see that $R m_{\lambda}$ is a nilpotent ideal. Hence, if either char $R \leq n$, or if $\mathbf{u}$ is not generic for $\mathscr{H}_{r, n}(\mathbf{u})$, then $\mathscr{H}_{r, n}(\mathbf{u})$ is not semisimple.

\section{$\S 7$. A cellular basis of $\mathscr{W}_{r, n}(\mathbf{u})$}

Throughout this section we assume that $R$ is a commutative ring in which 2 is invertible and that $\Omega$ is $\mathbf{u}$-admissible. This section constructs a cellular basis for $\mathscr{W}_{r, n}=\mathscr{W}_{r, n}(\mathbf{u})$ using the cellular bases of the algebras $\mathscr{H}_{r, n-2 f}=\mathscr{H}_{r, n-2 f}(\mathbf{u})$ for $0 \leq f \leq\left\lfloor\frac{n}{2}\right\rfloor$, together with a series of filtrations of $\mathscr{W}_{r, n}$. Our construction of a cellular basis of $\mathscr{W}_{r, n}$ is modelled on Enyang's work [Eny04] for the Brauer and BMW algebras.

Before we begin we need to fix some notation. Recall that the set $\left\{S_{1}, \ldots, S_{n-1}\right\}$ generates a subalgebra of $\mathscr{W}_{r, n}$ which is isomorphic to the group ring of $\mathfrak{S}_{n}$. For each permutation $w \in \mathfrak{S}_{n}$ we defined the corresponding braid diagram $\gamma(w)$ in Section 2 ; we now set $S_{w}=B_{\gamma(w)}$. Equivalently, if $w=\left(i_{1}, i_{1}+1\right) \cdots\left(i_{k}, i_{k}+1\right)$, where $1 \leq i_{j}<n$ for all $j$, then $S_{w}=S_{i_{1}} \cdots S_{i_{k}}$. Then $\left\{S_{w} \mid w \in \mathfrak{S}_{n}\right\}$ is a basis for the subalgebra of $\mathscr{W}_{r, n}$ generated by $\left\{S_{1}, \ldots, S_{n-1}\right\}$.

Next, suppose that $f$ is an integer with $0 \leq f \leq\left\lfloor\frac{n}{2}\right\rfloor$. It follows from Theorem 6.1 that we can identity $\mathscr{H}_{r, n-2 f}$ with the subalgebra of $\mathscr{H}_{r, n}$ generated by $Y_{i}$ and $T_{j}$, where $1 \leq i \leq n-2 f$ and $1 \leq j \leq n-2 f-1$. Similarly, by Proposition 5.6, we can identify $\mathscr{W}_{r, n-2 f}$ with the subalgebra of $\mathscr{W}_{r, n}$ generated by $X_{i}, S_{j}$ and $E_{j}$, where $1 \leq i \leq n-2 f$ and $1 \leq j \leq$ $n-2 f-1$.

Definition 7.1. Suppose $0 \leq f<\left\lfloor\frac{n}{2}\right\rfloor$. Let $\mathcal{E}_{f}=\mathscr{W}_{r, n-2 f} E_{1} \mathscr{W}_{r, n-2 f}$ be the two-sided ideal of $\mathscr{W}_{r, n-2 f}$ generated by $E_{1}$.

Proposition 7.2. Suppose that $0 \leq f<\left\lfloor\frac{n}{2}\right\rfloor$. Then there is a unique $R$-algebra isomorphism $\varepsilon_{f}: \mathscr{H}_{r, n-2 f} \cong \mathscr{W}_{r, n-2 f} / \mathcal{E}_{f}$ such that

$$
\varepsilon_{f}\left(T_{i}\right)=S_{i}+\mathcal{E}_{f} \quad \text { and } \quad \varepsilon_{f}\left(Y_{j}\right)=X_{j}+\mathcal{E}_{f},
$$


for $1 \leq i<n-2 f$ and $1 \leq j \leq n-2 f$.

Proof. We first show that $\mathscr{W}_{r, n-2 f} / \mathcal{E}_{f}$ is a free $R$-module of rank $r^{n-2 f}(n-2 f)$ !. It follows from the multiplication formulae for Brauer diagrams that an $r$-regular monomial $X^{\alpha} B_{\gamma} X^{\beta}$ in $\mathscr{W}_{r, n-2 f}$ belongs to $\mathcal{E}_{f}$ whenever $\gamma$ has a horizontal edge (equivalently, $\gamma \neq \gamma(w)$ for some $w \in$ $\left.\mathfrak{S}_{n-2 f}\right)$. If $\gamma=\gamma(w)$, for some $w \in \mathfrak{S}_{n-2 f}$, then $B_{\gamma}=S_{w}$ and $\gamma$ contains no horizontal edges, so the definition of regularity (Definition 2.9), forces $\beta=0$. So, by Theorem $5.5, \mathscr{W}_{r, n-2 f} / \mathcal{E}_{f}$ is spanned by the elements $\left\{X^{\alpha} S_{w}+\mathcal{E}_{f} \mid 0 \leq \alpha_{i}<r\right.$, for $1 \leq i \leq n-2 f$, and $\left.w \in \mathfrak{S}_{n-2 f}\right\}$. Note that this set contains $r^{n-2 f}(n-2 f)$ ! elements.

To see that the elements at the end of the last paragraph are linearly independent we use the seminormal representations from Section 4. Using the arguments and the notation from the proof of Theorem 5.5, it is enough to show that $\operatorname{dim}_{\mathbb{R}} \mathscr{W}_{\mathbb{R}}\left(\mathbf{u}^{\prime}\right) / \mathcal{E}_{f} \geq r^{n-2 f}(n-2 f)$ !. Now a seminormal representation $\Delta(\lambda)$ of $\mathscr{W}_{\mathbb{R}}\left(\mathbf{u}^{\prime}\right)$ is a representation of $\mathscr{W}_{\mathbb{R}}\left(\mathbf{u}^{\prime}\right) / \mathcal{E}_{f}$ if and only if $\mathcal{E}_{f} \Delta(\lambda)=0$, which happens if and only if $\lambda$ is a multipartition of $n-2 f$. Therefore, by the arguments of Section $5, \operatorname{dim}_{\mathbb{R}} \mathscr{W}_{\mathbb{R}}\left(\mathbf{u}^{\prime}\right) / \mathcal{E}_{f} \geq r^{n-2 f}(n-2 f)$ !. Hence, by the arguments used in the proof of Theorem 5.5 (compare, Theorem 6.1 , the elements above are a basis of $\mathscr{W}_{r, n-2 f} / \mathcal{E}_{f}$ and, consequently, $\mathscr{W}_{r, n-2 f} / \mathcal{E}_{f}$ is free as an $R$-module of rank $r^{n-2 f}(n-2 f)$ ! as claimed.

Inspecting the relations of $\mathscr{H}_{r, n-2 f}$ and $\mathscr{W}_{r, n-2 f}$ shows that there is a unique algebra homomorphism $\varepsilon_{f}: \mathscr{H}_{r, n-2 f} \rightarrow \mathscr{W}_{r, n-2 f} / \mathcal{E}_{f}$ such that $\varepsilon_{f}\left(T_{i}\right)=S_{i}+\mathcal{E}_{f}$ and $\varepsilon_{f}\left(Y_{j}\right)=X_{j}+\mathcal{E}_{f}$. To see that $\varepsilon_{f}$ is an isomorphism observe that $\varepsilon_{f}$ maps the basis of $\mathscr{H}_{r, n-2 f}$ to the basis of $\mathscr{W}_{r, n-2 f} / \mathcal{E}_{f}$. Hence, it is an isomorphism with inverse determined by $\varepsilon_{f}^{-1}\left(X^{\alpha} S_{w}+\mathcal{E}_{f}\right)=Y^{\alpha} T_{w}$, for $w \in \mathfrak{S}_{n-2 f}$ and $0 \leq \alpha_{i}<r$ where $1 \leq i \leq n-2 f$.

Definition 7.3. Let $E^{f}=E_{n-1} E_{n-3} \cdots E_{n-2 f+1}$ and let $\mathscr{W}_{r, n}^{f}=$ $\mathscr{W}_{r, n} E^{f} \mathscr{W}_{r, n}$ be the two-sided ideal of $\mathscr{W}_{r, n}$ generated by $E^{f}$. If $f=\left\lfloor\frac{n}{2}\right\rfloor$ then we set $\mathscr{H}_{r, n-2 f}=R$ and $\mathscr{W}_{r, n}^{f+1}=0$.

Note that this gives a filtration of $\mathscr{W}_{r, n}$ by two-sided ideals:

$$
\mathscr{W}_{r, n}=\mathscr{W}_{r, n}^{0} \supset \mathscr{W}_{r, n}^{1} \supset \cdots \supset \mathscr{W}_{r, n}^{\left\lfloor\frac{n}{2}\right\rfloor} \supset \mathscr{W}_{r, n}^{\left\lfloor\frac{n}{2}\right\rfloor+1}=0 .
$$

For $0 \leq f \leq\left\lfloor\frac{n}{2}\right\rfloor$ let $\pi_{f}: \mathscr{W}_{r, n}^{f} \rightarrow \mathscr{W}_{r, n}^{f} / \mathscr{W}_{r, n}^{f+1}$ be the corresponding projection map of $\mathscr{W}_{r, n}$-bimodules. 
For convenience we set $\mathbb{N}_{r}=\{0,1, \ldots, r-1\}$ and define $\mathbb{N}_{r}^{(f)}$ to be the set of $n$-tuples $\kappa=\left(k_{1}, \ldots, k_{n}\right)$ such that $k_{i} \in \mathbb{N}_{r}$ and $k_{i} \neq 0$ only for $i=n-1, n-3, \ldots, n-2 f+1$. Thus, if $\kappa \in \mathbb{N}_{r}^{(f)}$ then $X^{\kappa}=$ $X_{n-1}^{k_{n-1}} X_{n-3}^{k_{n-3}} \cdots X_{n-2 f+1}^{k_{n-2 f+1}} \in \mathscr{W}_{r, n}$.

LEMmA 7.4. Suppose that $0 \leq f \leq\left\lfloor\frac{n}{2}\right\rfloor$ and $\kappa \in \mathbb{N}_{r}^{(f)}$. Then $E^{f} X^{\kappa} \mathcal{E}_{f} \subset$ $\mathscr{W}_{r, n}^{f+1}$.

Proof. As $E^{f+1}=E^{f} E_{n-2 f-1}$, this follows because $\mathcal{E}_{f}=\mathscr{W}_{r, n-2 f}$ $E_{n-2 f-1} \mathscr{W}_{r, n-2 f}$ and every element of $\mathscr{W}_{r, n-2 f}$ commutes with $E^{f} X^{\kappa}$.

Combining the last two results we have a well-defined $R$-module homomorphism $\sigma_{f}: \mathscr{H}_{r, n-2 f} \rightarrow \mathscr{W}_{r, n}^{f} / \mathscr{W}_{r, n}^{f+1}$, for each integer $f$, with $0 \leq f \leq$ $\left\lfloor\frac{n}{2}\right\rfloor$, given by

$$
\sigma_{f}(h)=E^{f} \varepsilon_{f}(h)+\mathscr{W}_{r, n}^{f+1},
$$

for $h \in \mathscr{H}_{r, n-2 f}$.

We will need the following subgroups in order to understand the ideals $\mathscr{W}_{r, n}^{f}$.

Definition 7.5. Suppose that $0 \leq f \leq\left\lfloor\frac{n}{2}\right\rfloor$. Let $\mathfrak{B}_{f}$ be the subgroup of $\mathfrak{S}_{n}$ generated by $\left\{S_{n-1}, S_{n-2} S_{n-1} S_{n-3} S_{n-2}, \ldots, S_{n-2 f+2} S_{n-2 f+1}\right.$ $\left.S_{n-2 f+3} S_{n-2 f+2}\right\}$.

The symmetric group $\mathfrak{S}_{n}$ acts on the set of Brauer diagrams $\mathcal{B}(n)$ from the right. Let $\gamma=\gamma_{n-1} \circ \cdots \circ \gamma_{n-2 f+1}$. Then $E^{f}=B_{\gamma}$ and $\mathfrak{B}_{f}$ is the stabilizer in $\mathfrak{S}_{n}$ of the diagram $\gamma$. The group $\mathfrak{B}_{f}$ is isomorphic to the hyperoctahedral group $\mathbb{Z} / 2 \mathbb{Z} \imath \mathfrak{S}_{f}$, a Coxeter group of type $\mathfrak{B}_{f}$.

Given an integer $f$, with $0 \leq f \leq\left\lfloor\frac{n}{2}\right\rfloor$, let $\tau=\left((n-2 f),\left(2^{f}\right)\right)$ and define

$$
\mathcal{D}_{f}=\left\{\begin{array}{l|l}
d \in \mathfrak{S}_{n} & \begin{array}{c}
\mathfrak{t}^{\tau} d=\left(\mathfrak{t}_{1}, \mathfrak{t}_{2}\right) \text { is a row standard } \tau \text {-tableau and the first } \\
\text { column of } \mathfrak{t}_{2} \text { is increasing from top to bottom. }
\end{array}
\end{array}\right\}
$$

The following result is equivalent to [Eny04, Prop. 3.1]. (Enyang considers a subgroup of $\mathfrak{S}_{n}$ which is conjugate to $\mathfrak{B}_{f}$.)

Lemma 7.6. Suppose that $0 \leq f \leq\left\lfloor\frac{n}{2}\right\rfloor$. Then $\mathcal{D}_{f}$ is a complete set of right coset representatives for $\mathfrak{S}_{n-2 f} \times \mathfrak{B}_{f}$ in $\mathfrak{S}_{n}$. 
The point of introducing the subgroup $\mathfrak{B}_{f}$ is the following.

LEMmA 7.7. Suppose that $0 \leq f \leq\left\lfloor\frac{n}{2}\right\rfloor, w \in \mathfrak{S}_{n-2 f}$ and that $b \in \mathfrak{B}_{f}$. Then $S_{w} E^{f}=E^{f} S_{w}$ and $E^{f} S_{b}=E^{f}=S_{b} E^{f}$.

Proof. The first claim is obvious by $(2.1)(\mathrm{d})(\mathrm{i})$. For the second claim it is enough to consider the case when $b$ is a generator of $\mathfrak{B}_{f}$. In this case the claim is easily checked using the tangle relations and the untwisting relations.

Motivated by the definition of the elements $m_{\mathfrak{s t}} \in \mathscr{H}_{r, n-2 f}$ from the previous section, and by the work of Enyang [Eny04], we make the following definition.

Definition 7.8. Suppose that $0 \leq f \leq\left\lfloor\frac{n}{2}\right\rfloor$ and $\lambda \in \Lambda_{r}^{+}(n-2 f)$. Then for each pair $(\mathfrak{s}, \mathfrak{t})$ of standard $\lambda$-tableaux define

$$
M_{\mathfrak{s t}}=S_{d(\mathfrak{s})^{-1}} \cdot \prod_{s=2}^{r} \prod_{i=1}^{\left|\lambda^{(1)}\right|+\cdots+\left|\lambda^{(s-1)}\right|}\left(X_{i}-u_{s}\right) \sum_{w \in \mathfrak{S}_{\lambda}} S_{w} \cdot S_{d(\mathfrak{t})} .
$$

We remark that we will not ever really use this explicit formula for the elements $M_{\mathfrak{s t}}$. In what follows all that we need is a family of elements $\left\{M_{\mathfrak{s t}}\right\}$ in $\mathscr{W}_{r, n}$ which are related to some cellular basis of $\mathscr{H}_{r, n-2 f}$ as in Lemma $7.9(\mathrm{~d})$ below.

The following result follows easily using the relations of $\mathscr{W}_{r, n}$ and the definitions.

LEMmA 7.9. Suppose that $0 \leq f \leq\left\lfloor\frac{n}{2}\right\rfloor, \lambda \in \Lambda_{r}^{+}(n-2 f)$ and that $\mathfrak{s}, \mathfrak{t} \in \mathscr{T}^{\text {std }}(\lambda)$. Then:

a) $E^{f} M_{\mathfrak{s t}}=M_{\mathfrak{s t}} E^{f} \in \mathscr{W}_{r, n}^{f}$.

b) If $\kappa \in \mathbb{N}_{r}^{(f)}$ then $M_{\mathfrak{s t}} X^{\kappa}=X^{\kappa} M_{\mathfrak{s t}}$.

c) If $w$ is a permutation of $\{n-2 f+1, \ldots, n\}$ then $M_{\mathfrak{s t}} S_{w}=S_{w} M_{\mathfrak{s t}}$. In particular, $M_{\mathfrak{s t}} S_{w}=S_{w} M_{\mathfrak{s t}}$ if $w \in \mathfrak{B}_{f}$.

d) We have $\sigma_{f}\left(m_{\mathfrak{s t}}\right)=\pi_{f}\left(E^{f} M_{\mathfrak{s t}}\right)$.

The filtration of $\mathscr{W}_{r, n}$ given by the ideals $\mathscr{W}_{r, n}^{f}$ is still too coarse to be cellular. 
Definition 7.10. Suppose that $\lambda$ is a multipartition of $n-2 f$, where $0 \leq f \leq\left\lfloor\frac{n}{2}\right\rfloor$. Define $\mathscr{W}_{r, n} \stackrel{\unrhd \lambda}{ }$ to be the two-sided ideal of $\mathscr{W}_{r, n}$ generated by $\mathscr{W}_{r, n}^{f+1}$ and the elements

$$
\left\{E^{f} M_{\mathfrak{s t}} \mid \mathfrak{s}, \mathfrak{t} \in \mathscr{T}^{s t d}(\mu) \text { and } \mu \in \Lambda_{r}^{+}(n-2 f) \text { with } \mu \unrhd \lambda\right\} .
$$

We also set $\mathscr{W}_{r, n}^{\triangleright \lambda}=\sum_{\mu \triangleright \lambda} \mathscr{W}_{r, n}^{\unrhd \mu}$, where in the sum $\mu \in \Lambda_{r}^{+}(n-2 f)$.

Observe that

$$
\mathscr{W}_{r, n}^{f+1} \subseteq \mathscr{W}_{r, n}^{\triangleright \lambda} \subset \mathscr{W}_{r, n}^{\unrhd \lambda} \subseteq \mathscr{W}_{r, n}^{f}
$$

and that $\mathscr{W}_{r, n}^{\unrhd \lambda} \subset \mathscr{W}_{r, n}^{\triangleright \mu}$ whenever $\lambda \triangleright \mu$. Consequently, the ideals $\left\{\mathscr{W}_{r, n}^{\unrhd \lambda}\right\}$ give a refinement of the filtration of $\mathscr{W}_{r, n}$ by the ideals $\left\{\mathscr{W}_{r, n}^{f}\right\}$.

Definition 7.11. Suppose that $\mathfrak{s} \in \mathscr{T}^{\text {std }}(\lambda)$. We define $\Delta_{\mathfrak{s}}(f, \lambda)$ to be the $R$-submodule of $\mathscr{W}_{r, n}^{\unrhd \lambda} / \mathscr{W}_{r, n}^{\triangleright \lambda}$ spanned by the elements

$$
\left\{E^{f} M_{\mathfrak{s t}} X^{\kappa} S_{d}+\mathscr{W}_{r, n}^{\triangleright \lambda} \mid(\mathfrak{t}, \kappa, d) \in \delta(f, \lambda)\right\},
$$

where $\delta(f, \lambda)=\left\{(\mathfrak{t}, \kappa, d) \mid \mathfrak{t} \in \mathscr{T}^{s t d}(\lambda), \kappa \in \mathbb{N}_{r}^{(f)}\right.$ and $\left.d \in \mathcal{D}_{f}\right\}$.

We will see below that $\Delta_{\mathfrak{s}}(f, \lambda)$ is a right $\mathscr{W}_{r, n}$-module and that the spanning set in the definition is a basis of $\Delta_{\mathfrak{s}}(f, \lambda)$. Moreover, there is a natural isomorphism $\Delta_{\mathfrak{s}}(f, \lambda) \cong \Delta_{\mathfrak{t}}(f, \lambda)$, whenever $\mathfrak{s}, \mathfrak{t} \in \mathscr{T}^{\text {std }}(\lambda)$.

Before we begin studying the modules $\Delta_{\mathfrak{s}}(f, \lambda)$ it is convenient to define a degree function on $\mathscr{W}_{r, n}$. Recall from Theorem 5.5 that the set of $r$-regular monomials is a basis of $\mathscr{W}_{r, n}$.

Definition 7.12. Suppose that $a=\sum r_{\alpha \gamma \beta} X^{\alpha} B_{\gamma} X^{\beta} \in \mathscr{W}_{r, n}$, where each of the monomials in the sum is $r$-regular. Then the degree of $a$ is the integer

$$
\operatorname{deg} a=\max \left\{\sum_{i=1}^{n}\left(\alpha_{i}+\beta_{i}\right) \mid r_{\alpha \gamma \beta} \neq 0 \text { for some } \gamma \in \mathcal{B}(n)\right\}
$$

In particular, $\operatorname{deg} S_{i}=\operatorname{deg} E_{i}=0$, for $1 \leq i<n$, and $\operatorname{deg} X_{j}=1$, for $1 \leq j \leq n$. We note that the proof of [Naz96, Lemma 4.4] implies that

$$
\operatorname{deg}(a b) \leq \operatorname{deg}(a)+\operatorname{deg}(b), \quad \text { for all } a, b \in \mathscr{W}_{r, n}
$$


Lemma 7.13. Suppose that $1 \leq j<n$ and that $1 \leq k<r$. Then $E_{j} X_{j}^{k} E_{j}=E_{j} \omega_{j}^{(k)}$, where $\omega_{j}^{(k)}$ is a central element in $\mathscr{W}_{r, j-1}$ with $\operatorname{deg} \omega_{j}^{(k)}<$ $k$.

Proof. We argue by induction on $j$. If $j=1$ then $\operatorname{deg} \omega_{1}^{(k)}=0$ because $\omega_{1}^{(k)} \in R$ by relation $(2.1)(\mathrm{f})$. Suppose then that $j>1$.

By Lemma $4.15 \omega_{j}^{(k)}$ is a central element of $\mathscr{W}_{r, j-1}$ in $R\left[X_{1}, \ldots, X_{j-1}\right]$ and $\operatorname{deg} \omega_{j}^{(k)} \leq k$. Consequently, if $\omega_{j}^{(k)}=\sum_{\alpha} r_{\alpha} X^{\alpha}$, for some $r_{\alpha} \in R$, then $\omega_{j}^{(k)} E_{j}=\sum_{\alpha} r_{\alpha} X^{\alpha} E_{j}$ where each of the monomials $X^{\alpha} E_{j}$ is $r$-regular. Hence, $\operatorname{deg}\left(\omega_{j}^{(k)} E_{j}\right)=\operatorname{deg} \omega_{j}^{(k)}$. Therefore, it is enough to prove that $\operatorname{deg}\left(\omega_{j}^{(k)} E_{j}\right)<k$. By Lemma 2.3,

$$
\begin{aligned}
\omega_{j}^{(k)} E_{j} & =E_{j} X_{j}^{k} E_{j}=(-1)^{k} E_{j} X_{j+1}^{k} E_{j}=(-1)^{k} E_{j} S_{j-1} X_{j+1}^{k} S_{j-1} E_{j} \\
& =(-1)^{k} E_{j} E_{j-1} S_{j} X_{j+1}^{k} S_{j} E_{j-1} E_{j} \\
& =(-1)^{k} E_{j} E_{j-1}\left(X_{j}^{k}+X\right) E_{j-1} E_{j},
\end{aligned}
$$

where $X \in \mathscr{W}_{r, j+1}$ and $\operatorname{deg} X<k$ since $\operatorname{deg}(a b) \leq \operatorname{deg}(a)+\operatorname{deg}(b)$. We have that $\operatorname{deg}\left(E_{j} E_{j-1} X E_{j-1} E_{j}\right) \leq \operatorname{deg} X<k$ and that

$$
\begin{aligned}
E_{j} E_{j-1} X_{j}^{k} E_{j-1} E_{j} & =(-1)^{k} E_{j} E_{j-1} X_{j-1}^{k} E_{j-1} E_{j}=(-1)^{k} \omega_{j-1}^{(k)} E_{j} E_{j-1} E_{j} \\
& =(-1)^{k} \omega_{j-1}^{(k)} E_{j} .
\end{aligned}
$$

By induction $\operatorname{deg} \omega_{j-1}^{(k)}<k$, so this completes the proof of the Lemma.

Given integers $j$ and $k$, with $1 \leq j, k \leq n$, let $E_{j, k}=B_{\gamma}$ where $\gamma$ is the Brauer diagram with horizontal edges $\{j, k\}$ and $\{\bar{j}, \bar{k}\}$, and with all other edges being vertical. Thus, $S_{w} E_{i} S_{w^{-1}}=E_{(i) w^{-1},(i+1) w^{-1}}$, for all $w \in \mathfrak{S}_{n}$. Finally, note that $E_{i}=E_{i, i+1}$.

Until further notice we fix an integer $f$, with $0 \leq f \leq\left\lfloor\frac{n}{2}\right\rfloor$, a multipartition $\lambda \in \Lambda_{r}^{+}(n-2 f)$ and a standard $\lambda$-tableau $\mathfrak{s}$ and consider $\Delta(f, \lambda)=$ $\Delta_{\mathfrak{s}}(f, \lambda)$. The next two Lemmas show that $\Delta(f, \lambda)$ is a right $\mathscr{W}_{r, n}$-submodule of $\mathscr{W}_{r, n}^{\triangleright \lambda} / \mathscr{W}_{r, n}^{\triangleright \lambda}$ and that the action of $\mathscr{W}_{r, n}$ on $\Delta(f, \lambda)$ does not depend on $\mathfrak{s}$.

If $\kappa=\left(\kappa_{1}, \ldots, \kappa_{n}\right) \in \mathbb{N}_{r}^{(f)}$ we set $|\kappa|=\kappa_{n-1}+\kappa_{n-3}+\cdots+\kappa_{n-2 f+1}=$ $\operatorname{deg} X^{\kappa}$.

Lemma 7.14. Suppose that $\mathfrak{t} \in \mathscr{T}^{\text {std }}(\lambda)$ and $d \in \mathcal{D}_{f}$. For $1 \leq i<n$ and $1 \leq j \leq n$ there exist scalars $a_{\mathfrak{v} e}, b_{\mathfrak{v} e}, c_{\mathfrak{v} \rho e} \in R$, which do not depend on $\mathfrak{s}$, such that: 


$$
\begin{aligned}
& \text { a) } E^{f} M_{\mathfrak{s t}} S_{d} \cdot S_{i} \equiv \sum_{\substack{\mathfrak{v} \in \mathscr{T}^{s t d}(\lambda) \\
e \in \mathcal{D}_{f}}} a_{\mathfrak{v} e} E^{f} M_{\mathfrak{s v}} S_{e}\left(\bmod \mathscr{W}_{r, n}^{\triangleright \lambda}\right), \\
& \text { b) } E^{f} M_{\mathfrak{s t}} S_{d} \cdot E_{i} \equiv \sum_{\substack{\mathfrak{v} \in \mathscr{T}^{s t d}(\lambda) \\
e \in \mathcal{D}_{f}}} b_{\mathfrak{v} e} E^{f} M_{\mathfrak{s v}} S_{e}\left(\bmod \mathscr{W}_{r, n}^{\triangleright \lambda}\right), \\
& \text { c) } E^{f} M_{\mathfrak{s t}} S_{d} \cdot X_{j} \equiv \sum_{\substack{\mathfrak{v}, \rho, e) \in \delta(f, \lambda) \\
|\rho| \leq 1}} c_{\mathfrak{v} \rho e} E^{f} M_{\mathfrak{s v}} X^{\rho} S_{e}\left(\bmod \mathscr{W}_{r, n}^{\triangleright \lambda}\right) .
\end{aligned}
$$

Proof. (a) Now, $S_{d} S_{i}=S_{d(i, i+1)}$ and by Lemma 7.6 we can write $d(i, i+1)=a b e$ where $a \in \mathfrak{S}_{n-2 f}, b \in \mathfrak{B}_{f}$ and $e \in \mathcal{D}_{f}$; so $S_{d} S_{i}=S_{a} S_{b} S_{e}$. By part (d) of Lemma 7.9, respectively, we have

$$
E^{f} M_{\mathfrak{s t}} S_{a} \equiv E^{f} \varepsilon_{f}\left(m_{\mathfrak{s t}}\right) S_{a} \equiv E^{f} \varepsilon_{f}\left(m_{\mathfrak{s t}} T_{a}\right) \quad\left(\bmod \mathscr{W}_{r, n}^{\triangleright \lambda}\right)
$$

since $\mathscr{W}_{r, n}^{f+1} \subseteq \mathscr{W}_{r, n}^{\triangleright \lambda}$. As $m_{\mathfrak{s t}}$ is a cellular basis element for $\mathscr{H}_{r, n-2 f}$, we can write $m_{\mathfrak{s t}} T_{a}$ as a linear combination of terms $m_{\mathfrak{s v}}$ plus an element of $\mathscr{H}_{r, n}^{\triangleright \lambda}$. Consequently, $\left(E^{f} M_{\mathfrak{s t}}+\mathscr{W}_{r, n}^{\triangleright \lambda}\right) S_{a}$ can be written in the desired form. Hence, we may now assume that $a=1$.

To complete this case, observe that if $\mathfrak{v} \in \mathscr{T}^{s t d}(\lambda)$ then, by Lemma 7.9(c) and Lemma 7.7, $E^{f} M_{\mathfrak{s v}} S_{b} S_{e}=E^{f} S_{b} M_{\mathfrak{s v}} S_{e}=E^{f} M_{\mathfrak{s v}} S_{e}$ as required.

(b) We have to consider the product $E^{f} M_{\mathfrak{s t}} S_{d} E_{i}$. Let $j=(i) d^{-1}$ and $k=(i+1) d^{-1}$. Then $S_{d} E_{i}=E_{j, k} S_{d}$ so that $E^{f} M_{\mathfrak{s t}} S_{d} E_{i}=E^{f} M_{\mathfrak{s t}} E_{j, k} S_{d}$. By part (a) we may assume that $d=1$. We can also assume that $j<k$ since $E_{j, k}=E_{k, j}$. So we need to show that $E^{f} M_{\mathfrak{s t}} E_{j, k}+\mathscr{W}_{r, n}^{\triangleright \lambda}$ has the required form. There are three cases to consider.

(1) First, suppose that $j<k \leq n-2 f$. Then $E_{j, k} \in \mathscr{W}_{r, n-2 f}$, so that $M_{\mathfrak{s t}} E_{j, k} \in \mathcal{E}_{f}$ and $E^{f} M_{\mathfrak{s t}} E_{j, k} \in E^{f} \mathcal{E}_{f} \subseteq \mathscr{W}_{r, n}^{f+1}$ by Lemma 7.4. Hence, $E^{f} M_{\mathfrak{s t}} S_{d} E_{i} \in \mathscr{W}_{r, n}^{f+1} \subseteq \mathscr{W}_{r, n}^{\triangleright \lambda}$ and part (b) is true when $j<k \leq n-2 f$.

(2) Next, suppose that $j \leq n-2 f<k$. An easy exercise in multiplying Brauer diagrams shows that

$$
E^{f} E_{j, k}= \begin{cases}E^{f} S_{(j, k-1)}, & \text { if } n-k \text { is even, } \\ E^{f} S_{(j, k+1)}, & \text { if } n-k \text { is odd }\end{cases}
$$

So Lemma 7.9 (a) implies that $E^{f} M_{\mathfrak{s t}} S_{d} E_{i}=M_{\mathfrak{s t}} E^{f} E_{j, k} S_{d}=E^{f} M_{\mathfrak{s t}} S_{d}$ $S_{d^{-1}(j, k \pm 1) d}$, we again deduce the result from part (a). 
(3) Finally, suppose that $n-2 f<j<k$. Then $M_{\mathfrak{s t}} E_{j, k}=E_{j, k} M_{\mathfrak{s t}}$ and a Brauer diagram calculation shows that $E^{f} E_{j, k}=E^{f} S_{w}$, where $w$ is a permutation of $\{n-2 f+1, \ldots, n\}$. Consequently,

$$
\begin{aligned}
E^{f} M_{\mathfrak{s t}} S_{d} E_{i} & =E^{f} M_{\mathfrak{s t}} E_{j, k} S_{d}=E^{f} E_{j, k} M_{\mathfrak{s t}} S_{d} \\
& =E^{f} S_{w} M_{\mathfrak{s t}} S_{d}=E^{f} M_{\mathfrak{s t}} S_{w} S_{d}
\end{aligned}
$$

where the last equality follows from Lemma 7.9(c). As $S_{w} S_{d}=S_{d} S_{d^{-1} w d}$ we are done by part (a).

(c) It follows from the skein relations that $S_{d} X_{j}=X_{(j) d} S_{d}+B$, for some $B \in \mathscr{B}_{n}\left(\omega_{0}\right)$. Hence, by parts (a) and (b) it suffices to show that $E^{f} M_{\mathfrak{s t}} X_{i}$ can be written in the required form, for $1 \leq i \leq n$. If $i \leq n-2 f$ then

$$
E^{f} M_{\mathfrak{s t}} X_{i}+\mathscr{W}_{r, n}^{\triangleright \lambda}=E^{f} \varepsilon_{f}\left(m_{\mathfrak{s t}}\right) X_{i}+\mathscr{W}_{r, n}^{\triangleright \lambda}=E^{f} \varepsilon_{f}\left(m_{\mathfrak{s t}} Y_{i}\right)+\mathscr{W}_{r, n}^{\triangleright \lambda},
$$

so the result follows because $m_{\mathfrak{s t}}$ is a cellular basis element of $\mathscr{H}_{r, n-2 f}$. If $i>n-2 f$ then the result is immediate if $n-i$ is odd. If $n-i$ is even then $i-1>n-2 f$, so the result follows because $E_{i-1} X_{i}=-E_{i-1} X_{i-1}$ by $(2.1)(\mathrm{h})$.

This completes the proof of the Lemma.

Proposition 7.15. Suppose that $(\mathfrak{t}, \kappa, d) \in \delta(f, \lambda)$. For $1 \leq i<n$ and $1 \leq j \leq n$ there exist scalars $a_{\mathfrak{v} \rho e}, b_{\mathfrak{v} \rho e}, c_{\mathfrak{v} \rho e} \in R$, which do not depend on $\mathfrak{s}$, such that:

a) $E^{f} M_{\mathfrak{s t}} X^{\kappa} S_{d} \cdot S_{i} \equiv \sum_{\substack{(\mathfrak{v}, \rho, e) \in \delta(f, \lambda) \\|\rho| \leq|\kappa|}} a_{\mathfrak{v} \rho e} E^{f} M_{\mathfrak{s b}} X^{\rho} S_{e}\left(\bmod \mathscr{W}_{r, n}^{\triangleright \lambda}\right)$,

b) $E^{f} M_{\mathfrak{s t}} X^{\kappa} S_{d} \cdot E_{i} \equiv \sum_{\substack{(\mathfrak{v}, \rho, e) \in \delta(f, \lambda) \\|\rho| \leq|\kappa|}} b_{\mathfrak{v} \rho e} E^{f} M_{\mathfrak{s v}} X^{\rho} S_{e}\left(\bmod \mathscr{W}_{r, n}^{\triangleright \lambda}\right)$,

c) $E^{f} M_{\mathfrak{s t}} X^{\kappa} S_{d} \cdot X_{j} \equiv \sum_{\substack{(\mathfrak{v}, \rho, e) \in \delta(f, \lambda) \\|\rho| \leq|\kappa|+1}} c_{\mathfrak{v} \rho e} E^{f} M_{\mathfrak{s v}} X^{\rho} S_{e}\left(\bmod \mathscr{W}_{r, n}^{\triangleright \lambda}\right)$

Proof. The case $|\kappa|=0$ is precisely Lemma 7.14. We now assume that $|\kappa|>0$ and argue by induction on $|\kappa|$.

(a) Write $S_{d} S_{i}=S_{a} S_{b} S_{e}$, where $a \in \mathfrak{S}_{n-2 f}, b \in \mathfrak{B}_{f}$ and $e \in \mathcal{D}_{f}$. As $E^{f} M_{\mathfrak{s t}} X^{\kappa}=E^{f} X^{\kappa} M_{\mathfrak{s t}}$ we may assume that $a=1$ by repeating the 
argument from the proof part (a) of Lemma 7.14. By the right handed version of Lemma $2.3, X^{\kappa} S_{b}=S_{b} X^{\kappa b^{-1}}+X$, where $X$ is a linear combination of monomials of the form $x_{1} \cdots x_{k}$ with $x_{j} \in\left\{S_{l}, E_{l}, X_{m} \mid 1 \leq\right.$ $l<n$ and $1 \leq m \leq n\}$ and $k<|\kappa|$. For each summand $x_{1} \cdots x_{k}$ of $X$ we have $k<|\kappa|$ so by induction we can write $\left(E^{f} M_{\mathfrak{s t}}+\mathscr{W}_{r, n}^{\triangleright \lambda}\right) x_{1} \cdots x_{l}$ in the required form, for $l=1, \ldots, k$; consequently, by induction, we can write $\left(E^{f} M_{\mathfrak{s t}}+\mathscr{W}_{r, n}^{\triangleright \lambda}\right) x_{1} \cdots x_{k} S_{e}$ in the required form. Hence, we are reduced to showing that $E^{f} M_{\mathfrak{s t}} S_{b} X^{\kappa b^{-1}} S_{e}+\mathscr{W}_{r, n}^{\triangleright \lambda}$ can be written in the required form. Now, $E^{f} M_{\mathfrak{s t}} S_{b}=E^{f} S_{b} M_{\mathfrak{s t}}=E^{f} M_{\mathfrak{s t}}$ by Lemma $7.9(\mathrm{c})$ and Lemma 7.7. Therefore, using Lemma 7.7 once again,

$$
\begin{aligned}
E^{f} M_{\mathfrak{s t}} S_{b} X^{\kappa b^{-1}} S_{e} & =E^{f} M_{\mathfrak{s t}} X^{\kappa b^{-1}} S_{e}=M_{\mathfrak{s t}} E^{f} X^{\kappa b^{-1}} S_{e} \\
& = \pm M_{\mathfrak{s t}} E^{f} X^{\kappa^{\prime}} S_{e}
\end{aligned}
$$

where $\kappa^{\prime} \in \mathbb{N}_{r}^{(f)}$ because $b \in B^{f}$ and $E_{j} X_{j+1}=-E_{j} X_{j}$ by the skein relations. Hence, $E^{f} M_{\mathfrak{s t}} S_{b} X^{\kappa b^{-1}} S_{e}= \pm E^{f} M_{\mathfrak{s t}} X^{\kappa^{\prime}} S_{e}$ and the inductive step of the Proposition is proved when $h=S_{i}$.

(b) As in the proof of part (b) of Lemma 7.14, we have $E^{f} M_{\mathfrak{s t}} X^{\kappa} S_{d} E_{i}=$ $E^{f} M_{\mathfrak{s t}} X^{\kappa} E_{j, k} S_{d}$, where $j=(i) d^{-1}$ and $k=(i+1) d^{-1}$. Further, as $E_{j, k}=$ $E_{k, j}$ we may assume that $j<k$ and, by part (a), we may assume that $d=1$. So we need to show that $E^{f} M_{\mathfrak{s t}} X^{\kappa} E_{j, k}+\mathscr{W}_{r, n}^{\triangleright \lambda}$ has the required form. There are two cases to consider.

Case b1. $k=j+1$ : We must show that $E^{f} M_{\mathfrak{s t}} X^{\kappa} E_{j}$ can be written in the required form.

First suppose that $j<n-2 f$. Then we may repeat the argument from the proof of part (b) of Lemma 7.14 to see that $M_{\mathfrak{s t}} E_{j} \in \mathcal{E}_{f}$, so that

$$
E^{f} M_{\mathfrak{s t}} X^{\kappa} E_{j}=E^{f} X^{\kappa} M_{\mathfrak{s t}} E_{j} \in E^{f} X^{\kappa} \mathcal{E}_{f}
$$

Hence, $E^{f} M_{\mathfrak{s t}} X^{\kappa} S_{d} E_{i} \in \mathscr{W}_{r, n}^{f+1} \subseteq \mathscr{W}_{r, n}^{\triangleright \lambda}$ by Lemma 7.4, and the Proposition is true when $j<n-2 f$.

Next, suppose that $j \geq n-2 f$. If $\kappa_{j}+\kappa_{j+1}=0$ then $X^{\kappa} E_{j}=E_{j} X^{\kappa}$ so the result follows by induction. Suppose then that $\kappa_{j}+\kappa_{j+1}>0$.

If $j \equiv n-1(\bmod 2)$ then $E_{j}$ is a factor of $E_{f}$ and $\kappa_{j}>0$. By Lemma 7.13 we have that $E_{j} X_{j}^{\kappa_{j}} E_{j}=E_{j} \omega_{j}^{\left(\kappa_{j}\right)}$, where $\omega_{j}^{\left(\kappa_{j}\right)}$ is a central element of $\mathscr{W}_{r, j-1}$ with $\operatorname{deg} \omega_{j}^{\left(\kappa_{j}\right)}<\kappa_{j}$. Write $E^{f}=\dot{E}^{f} E_{j}$ and $X^{\kappa}=\dot{X}^{\kappa} X_{j}^{\kappa_{j}}$. 
Then

$$
\begin{aligned}
E^{f} M_{\mathfrak{s t}} X^{\kappa} E_{j} & =\dot{E}^{f} M_{\mathfrak{s t}} \dot{X}^{\kappa} E_{j} X_{j}^{\kappa_{j}} E_{j}=\dot{E}^{f} M_{\mathfrak{s t}} \dot{X}^{\kappa} E_{j} \omega_{j}^{\left(\kappa_{j}\right)} \\
& =E^{f} M_{\mathfrak{s t}} \dot{X}^{\kappa} E_{j} \omega_{j}^{\left(\kappa_{j}\right)}
\end{aligned}
$$

As $\operatorname{deg} \dot{X}^{\kappa}=|\kappa|-\kappa_{j}$ and $\operatorname{deg} \omega_{j}^{\left(\kappa_{j}\right)}<\kappa_{j}$, the result now follows by writing $\omega_{j}^{\left(\kappa_{j}\right)}$ as a linear combination of terms of the form $x_{1} \cdots x_{l}$ and applying induction to each of the products $E^{f} M_{\mathfrak{s t}} \dot{X}^{\kappa} E_{j} x_{1} \cdots x_{m}$, for $1 \leq m \leq l$ (compare the proof of part (a)).

If $j \equiv n(\bmod 2)$ then $E_{j+1}$ is a factor of $E^{f}$ and $\kappa_{j+1}>0$. Write $E^{f}=\dot{E}^{f} E_{j+1}$ and $X^{\kappa}=\dot{X}^{\kappa} X_{j+1}^{\kappa j+1}$. Then

$$
\begin{aligned}
E^{f} M_{\mathfrak{s t}} X^{\kappa} E_{j} & =\dot{E}^{f} M_{\mathfrak{s t}} \dot{X}^{\kappa} E_{j+1} X_{j+1}^{\kappa_{j+1}} E_{j}= \pm \dot{E}^{f} M_{\mathfrak{s t}} \dot{X}^{\kappa} E_{j+1} X_{j}^{\kappa_{j+1}} E_{j} \\
& = \pm \dot{E}^{f} M_{\mathfrak{s t}} \dot{X}^{\kappa} X_{j}^{\kappa_{j+1}} E_{j+1} E_{j}= \pm \dot{E}^{f} M_{\mathfrak{s t}} \dot{X}^{\kappa} X_{j}^{\kappa_{j+1}} E_{j+1} S_{j} S_{j+1} \\
& = \pm \dot{E}^{f} M_{\mathfrak{s t}} \dot{X}^{\kappa} E_{j+1} X_{j}^{\kappa_{j+1}} S_{j} S_{j+1}=E^{f} M_{\mathfrak{s t}} X^{\kappa} S_{j} S_{j+1} .
\end{aligned}
$$

Hence, the result follows by part (a).

Case b2. $k>j+1$ : Since $|\kappa|>0$ we can fix $l$ with $\kappa_{l} \neq 0$ (so $l \equiv n-1$ $(\bmod 2))$. Write $E^{f}=\dot{E}^{f} E_{l}$ and $X^{\kappa}=\dot{X}^{\kappa} X_{l}^{\kappa_{l}}$. Set $l^{\prime}=l$ if $l \notin\{j, k\}$ and $l^{\prime}=l+1$ if $l \in\{j, k\}$, and put $l^{\prime \prime}=l^{\prime}$ if $l^{\prime} \neq j+1$ and $l^{\prime \prime}=k$ if $l^{\prime}=j+1$. Note that $l^{\prime} \notin\{j, k\}$ and $l^{\prime \prime} \notin\{j, j+1\}$ since $k>j+1$. We have

$$
\begin{aligned}
E^{f} M_{\mathfrak{s t}} X^{\kappa} E_{j, k} & = \pm \dot{E}^{f} M_{\mathfrak{s t}} \dot{X}^{\kappa} E_{l} X_{l^{\prime}}^{\kappa_{l}} S_{(j+1, k)} E_{j} S_{(j+1, k)} \\
& = \pm \dot{E}^{f} M_{\mathfrak{s t}} \dot{X}^{\kappa} E_{l}\left(S_{(j+1, k)} X_{l^{\prime \prime}}^{\kappa_{l}}+X\right) E_{j} S_{(j+1, k)} \\
& = \pm E^{f} M_{\mathfrak{s t}} \dot{X}^{\kappa}\left(S_{(j+1, k)} X_{l^{\prime \prime}}^{\kappa_{l}}+X\right) E_{j} S_{(j+1, k)}
\end{aligned}
$$

where $\operatorname{deg} X<\kappa_{l}$. Hence, by induction and part (a) it suffices to show that $E^{f} M_{\mathfrak{s t}} \dot{X}^{\kappa} S_{(j+1, k)} X_{l^{\prime \prime}}^{\kappa_{l}} E_{j}$ can be written in the required form. As $l^{\prime \prime} \notin$ $\{j, j+1\}$

$$
E^{f} M_{\mathfrak{s t}} \dot{X}^{\kappa} S_{(j+1, k)} X_{l^{\prime \prime}}^{\kappa_{l}} E_{j}=E^{f} M_{\mathfrak{s t}} \dot{X}^{\kappa} S_{(j+1, k)} E_{j} X_{l^{\prime \prime}}^{\kappa_{l}}
$$

Therefore, $E^{f} M_{\mathfrak{s t}} X^{\kappa} E_{j, k}$ can be written in the required form by induction.

(c) As in the proof of Lemma 7.14, we may assume that $r>1$ and, by the skein relations, $S_{d} X_{j}=X_{(j) d} S_{d}+B$, for some $B \in \mathscr{B}_{n}\left(\omega_{0}\right)$. Hence, by 
parts (a) and (b) it suffices to show that $E^{f} M_{\mathfrak{s t}} X^{\kappa} \cdot X_{i}$ can be written in the required form. If $i \leq n-2 f$ then

$$
\begin{aligned}
E^{f} M_{\mathfrak{s t}} X^{\kappa} X_{i}+\mathscr{W}_{r, n}^{\triangleright \lambda} & =E^{f} X^{\kappa} \sigma\left(m_{\mathfrak{s t}}\right) X_{i}+\mathscr{W}_{r, n}^{\triangleright \lambda} \\
& =E^{f} X^{\kappa} \sigma\left(m_{\mathfrak{s t}} Y_{i}\right)+\mathscr{W}_{r, n}^{\triangleright \lambda}
\end{aligned}
$$

so the result follows because $\left\{m_{\mathfrak{s t}}\right\}$ is a cellular basis of $\mathscr{H}_{r, n-2 f}$. If $i>n-2 f$ and $\kappa_{i}<r-1$ then $E^{f} M_{\mathfrak{s t}} X^{\kappa} X_{i}$ is of the desired form. If $\kappa_{i}=r-1$ then $X_{i}^{\kappa_{i}} X_{i}=X_{i}^{r}$ can be written as a linear combination of $r$-regular monomials of degree less than or equal to $\kappa_{i}$ by the proof of Theorem 5.5. Hence, using parts (a) and (b) and induction for each of these $r$-regular monomials, $E^{f} M_{\mathfrak{s t}} X^{\kappa} X_{i}+\mathscr{W}_{r, n}^{\triangleright \lambda}$ can be written in the required form.

This completes the proof of the Proposition.

Recall from $(2.2)$ that $\mathscr{W}^{\text {aff }}$ has a unique anti-automorphism $*: \mathscr{W}^{\text {aff }} \rightarrow$ $\mathscr{W}^{\text {aff }}$ which fixes all of the generators of $\mathscr{W}^{\text {aff }}$. This involution induces an anti-isomorphism of $\mathscr{W}_{r, n}$, which we also call $*$. Thus, $S_{i}^{*}=S_{i}, E_{i}^{*}=E_{i}$, $X_{j}^{*}=X_{j}$ and $(a b)^{*}=b^{*} a^{*}$, for $1 \leq i<n, 1 \leq j \leq n$ and all $a, b \in \mathscr{W}_{r, n}$. Observe that $S_{w}^{*}=S_{w^{-1}}$, for $w \in \mathfrak{S}_{n}$, and that $M_{\mathfrak{s t}}^{*}=M_{\mathfrak{t s}}$.

Proposition 7.16. Suppose $0 \leq f \leq\left\lfloor\frac{n}{2}\right\rfloor$ and $\lambda \in \Lambda_{r}^{+}(n-2 f)$. Then $\mathscr{W}_{r, n}^{\unrhd \lambda} / \mathscr{W}_{r, n}^{\triangleright \lambda}$ is spanned by the elements

$$
\left\{S_{e}^{*} X^{\rho} E^{f} M_{\mathfrak{s t}} X^{\kappa} S_{d}+\mathscr{W}_{r, n}^{\triangleright \lambda} \mid(\mathfrak{t}, \kappa, d),(\mathfrak{s}, \rho, e) \in \delta(f, \lambda)\right\} .
$$

Proof. Let $W$ be the $R$-submodule of $\mathscr{W}_{r, n}^{\unrhd \lambda} / \mathscr{W}_{r, n}^{\triangleright \lambda}$ spanned by the elements in the statement of the Proposition. As the generators $\left\{E^{f} M_{\mathfrak{s t}}+\right.$ $\left.\mathscr{W}_{r, n}^{\triangleright \lambda}\right\}$ of $\mathscr{W}_{r, n}^{\unrhd \lambda} / \mathscr{W}_{r, n}^{\triangleright \lambda}$ are contained in $W$, and $W \subseteq \mathscr{W}_{r, n}^{\unrhd \lambda} / \mathscr{W}_{r, n}^{\triangleright \lambda}$, it suffices to show that $W$ is a $\mathscr{W}_{r, n}$-bimodule.

First, by Proposition 7.15, $W$ is closed under right multiplication by elements of $\mathscr{W}_{r, n}$. To see that $W$ is also closed under left multiplication by elements of $\mathscr{W}_{r, n}$ note that $\left(\mathscr{W}_{r, n}^{\triangleright \lambda}\right)^{*}=\mathscr{W}_{r, n}^{\triangleright \lambda}$ as the set of generators for $\mathscr{W}_{r, n}^{\triangleright \lambda}$ is invariant under $*$ because $\left(E^{f} M_{\mathfrak{s t}}\right)^{*}=M_{\mathfrak{t s}}\left(E^{f}\right)^{*}=M_{\mathfrak{t s}} E^{f}=E^{f} M_{\mathfrak{t s}}$. Therefore, if $a \in \mathscr{W}_{r, n}$ then

$$
a\left(S_{e}^{*} X^{\rho} E^{f} M_{\mathfrak{s t}} X^{\kappa} S_{d}+\mathscr{W}_{r, n}^{\triangleright \lambda}\right)=\left(\left(S_{d}^{*} X^{\kappa} E^{f} M_{\mathfrak{t s}} X^{\rho} S_{e}+\mathscr{W}_{r, n}^{\triangleright \lambda}\right) a^{*}\right)^{*} \in W
$$

by Proposition 7.15. Hence, $W$ is closed under left multiplication by elements of $\mathscr{W}_{r, n}$. 
Let $\Lambda_{r}^{+}=\left\{(f, \lambda) \mid 0 \leq f \leq\left\lfloor\frac{n}{2}\right\rfloor\right.$ and $\left.\lambda \in \Lambda_{r}^{+}(n-2 f)\right\}$. If $(f, \lambda) \in \Lambda_{r}^{+}$ and $(\mathfrak{s}, \rho, e),(\mathfrak{t}, \kappa, d) \in \delta(f, \lambda)$ then we define

$$
C_{(\mathfrak{s}, \rho, e)(\mathfrak{t}, \kappa, d)}^{(f, \lambda)}=S_{e}^{*} X^{\rho} E^{f} M_{\mathfrak{s t}} X^{\kappa} S_{d} .
$$

We can now prove Theorem B from the introduction.

THEOREM 7.17. Let $R$ be a commutative ring in which 2 is invertible and let $\mathbf{u} \in R^{r}$. Suppose that $\Omega$ is $\mathbf{u}$-admissible. Then

$$
\mathscr{C}=\left\{C_{(\mathfrak{s}, \rho, e)(\mathfrak{t}, \kappa, d)}^{(f, \lambda)} \mid(\mathfrak{s}, \rho, e),(\mathfrak{t}, \kappa, d) \in \delta(f, \lambda), \text { where }(f, \lambda) \in \Lambda_{r}^{+}\right\}
$$

is a cellular basis of $\mathscr{W}_{r, n}(\mathbf{u})$.

Proof. Applying the definitions it is easy to check that $\left(C_{(\mathfrak{s}, \rho, e)(\mathfrak{t}, \kappa, d)}^{(f, \lambda)}\right)^{*}=$ $C_{(\mathfrak{t}, \kappa, d)(\mathfrak{s}, \rho, e)}^{(f, \lambda)}$. Furthermore, by Proposition 7.15, for each $h \in \mathscr{W}_{r, n}$ there exist scalars $r_{\left(\mathfrak{t}^{\prime}, \kappa^{\prime}, d^{\prime}\right)}(h) \in R$, which do not depend on $(\mathfrak{s}, \rho, e)$, such that

$$
C_{(\mathfrak{s}, \rho, e)(\mathfrak{t}, \kappa, d)}^{(f, \lambda)} \cdot h=\sum_{\left(\mathfrak{t}^{\prime}, \kappa^{\prime}, d^{\prime}\right) \in \delta(f, \lambda)} r_{\left(\mathfrak{t}^{\prime}, \kappa^{\prime}, d^{\prime}\right)}(h) C_{(\mathfrak{s}, \rho, e)\left(\mathfrak{t}^{\prime}, \kappa^{\prime}, d^{\prime}\right)}^{(f, \lambda)} \quad\left(\bmod \mathscr{W}_{r, n}^{\triangleright \lambda}\right) .
$$

To show that $\mathscr{C}$ is a cellular basis of $\mathscr{W}_{r, n}$ it remains to check that $\mathscr{C}$ is a basis of $\mathscr{W}_{r, n}$. Now, $\mathscr{W}_{r, n}=\mathscr{W}_{r, n}^{0} \supset \mathscr{W}_{r, n}^{1} \supset \cdots \supset \mathscr{W}_{r, n}^{\left\lfloor\frac{n}{2}\right\rfloor}$ is a filtration of $\mathscr{W}_{r, n}$ by two-sided ideals, and the two-sided ideals $\mathscr{W}_{r, n}^{\unrhd \lambda}$, where $\lambda \in \Lambda_{r}^{+}(n-2 f)$, induce a filtration of $\mathscr{W}_{r, n}^{f} / \mathscr{W}_{r, n}^{f+1}$. Therefore, $\mathscr{C}$ spans $\mathscr{W}_{r, n}$ by Proposition 7.16. To complete the proof observe that $\# \delta(f, \lambda)=\# \mathscr{T}_{n}^{u d}(\lambda)$, by

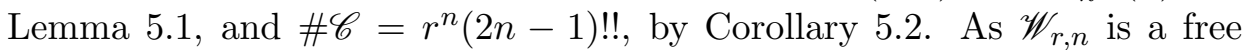
$R$-module of rank $r^{n}(2 n-1)$ !! by Theorem 5.5 , this implies that $\mathscr{C}$ is an $R$-basis of $\mathscr{W}_{r, n}$. Hence, $\mathscr{C}$ is a cellular basis of $\mathscr{W}_{r, n}$ as required.

The reader may check that the proof of Theorem 7.17 does not rely on the explicit definition of the elements $M_{\mathfrak{s t}} \in \mathscr{W}_{t, n}(\mathbf{u})$. The important property of these elements, as far as the proof of the Theorem is concerned, is that they are related to a cellular basis of $\mathscr{H}_{r, n}(\mathbf{u})$ by the formula of Lemma 7.9(d). Consequently, for each cellular basis of $\mathscr{H}_{r, n}(\mathbf{u})$ the argument of Theorem 7.17 produces a corresponding cellular basis of $\mathscr{W}_{r, n}(\mathbf{u})$.

We now show that we can, in principle, construct all of the finite dimensional irreducible representations of the affine Wenzl algebras over an 
algebraically closed field. First recall that $\Omega$ is rational if for $i \gg 0$ it satisfies a recurrence relation of the form $\omega_{i+k}+a_{1} \omega_{i+k-1}+\cdots+a_{k} \omega_{i}=0$, for some $k>0$ and some $a_{1}, \ldots, a_{k} \in R$.

LEMMA 7.18. Suppose that $\Omega$ is admissible and that $R$ is algebraically closed. Then $\Omega$ is rational if and only if there is a finite dimensional $\mathscr{W}_{n}^{\text {aff }}(\Omega)$-module upon which $E_{1}$ is non-zero.

Proof. First suppose that $\Omega$ is rational. As in the proof of Proposition $3.11, \Omega$ is rational if and only if

$$
\widetilde{W}_{1}(y)+y-\frac{1}{2}=\left(y+\frac{1}{2}\right) \prod_{i=1}^{s} \frac{y+c_{i}}{y-c_{i}},
$$

for some $c_{i} \in R$ and some $s \geq 0$. Hence, if $\Omega$ is rational then $\Omega$ is $\mathbf{u}^{-}$ admissible where

$$
\mathbf{u}= \begin{cases}\left(c_{1}, \ldots, c_{s}\right), & \text { if } s \text { is odd } \\ \left(c_{1}, \ldots, c_{s}, 0\right), & \text { if } s \text { is even }\end{cases}
$$

Hence, $\mathscr{W}_{r, n}(\mathbf{u})$ is a finite dimensional $\mathscr{W}_{n}^{\text {aff }}(\Omega)$-module upon which the action of $E_{1}$ is non-zero.

Conversely, suppose that there is a finite dimensional $\mathscr{W}_{n}^{\text {aff }}(\mathbf{u})$-module $M$ upon which $E_{1}$ is non-zero. Let $c(t)=\operatorname{det}\left(t I-X_{1}\right)$ be the characteristic polynomial for $X_{1}$ acting on $M$, where $t$ is an indeterminate and $I$ is the identity matrix on $M$. Write $c(t)=\sum_{j=0}^{k} a_{j} t^{k-j}$, where $a_{0}=1$. Then $\sum_{j=0}^{k} a_{j} X_{1}^{k-j}=0$ on $M$ by the Cayley-Hamilton theorem. Hence, $\sum_{j=0}^{k} a_{j} E_{1} X_{1}^{i+k-j} E_{1}=\sum_{j=0}^{k} a_{j} \omega_{i+k-j} E_{1}$ is zero on $M$, for any $i \geq 0$. Therefore, $\omega_{i+k}+a_{1} \omega_{i+k-1}+\cdots+a_{k} \omega_{i}=0$, for $i \geq 0$, since $E_{1}$ is non-zero on $M$. Thus, $\Omega$ is rational as required.

THEOREM 7.19. Suppose that $R$ is an algebraically closed field. Then we can construct all of the finite dimensional irreducible $\mathscr{W}_{n}^{a f f}(\Omega)$-modules.

Proof. First suppose that $\mathscr{W}_{n}^{\text {aff }}(\Omega)$ has a finite dimensional irreducible module upon which $E_{1}$ is non-zero. Then $\Omega$ is admissible. Then $\Omega$ is rational by Lemma 7.18. Hence, by Proposition 3.11 every finite dimensional irreducible $\mathscr{W}_{n}^{\text {aff }}(\Omega)$-module can be considered as a finite dimensional $\mathscr{W}_{r, n}(\mathbf{u})$-module for some $\mathbf{u} \in R^{r}$ such that $\Omega$ is $\mathbf{u}$-admissible. By Theorem $7.17 \mathscr{W}_{r, n}(\mathbf{u})$ is a cellular algebra, so every irreducible $\mathscr{W}_{r, n}(\mathbf{u})$-module 
arises (in a unique way) as the head of some cell module. Hence, we have a construction of every finite dimensional irreducible $\mathscr{W}_{n}^{\text {aff }}(\Omega)$-module when $\Omega$ is rational.

Finally, suppose that $E_{1}$ acts as zero on every finite dimensional irreducible $\mathscr{W}_{n}^{\text {aff }}(\Omega)$-module. Then every finite dimensional irreducible module $M$ can be considered as an irreducible module for the degenerate affine Hecke algebra of type $A$. Therefore, $M$ can be considered as an irreducible module for some degenerate Hecke algebra $\mathscr{H}_{r, n}(\mathbf{u})$ (cf. the proof of Proposition 3.11). Now $\mathscr{H}_{r, n}(\mathbf{u})$ is a cellular algebra by Theorem 6.3 , so we can again construct all finite dimensional $\mathscr{W}_{n}^{\text {aff }}(\Omega)$-modules.

Note that any given irreducible $\mathscr{W}_{n}^{\text {aff }}(\Omega)$-module can be considered as an irreducible module for an infinite number of cyclotomic Nazarov-Wenzl algebras. Consequently, the classification of the irreducible $\mathscr{W}_{r, n}(\mathbf{u})$-modules when $\omega_{0} \neq 0$ (Theorem $\mathrm{C}$ ), does not give a classification of the finite dimensional irreducible $\mathscr{W}_{n}^{\text {aff }}(\Omega)$-modules when $\Omega$ is admissible and $\omega_{0} \neq 0$.

\section{§8. Classification of the irreducible $\mathscr{W}_{r, n}(\mathbf{u})$-modules}

In this section we classify the irreducible $\mathscr{W}_{r, n}(\mathbf{u})$-modules, for fields in which 2 is invertible, in terms of the irreducible $\mathscr{H}_{r, n}(\mathbf{u})$-modules. As the involution $*$ induces a functorial bijection between left $\mathscr{W}_{r, n}$-modules and right $\mathscr{W}_{r, n}$-modules, we continue to work with right $\mathscr{W}_{r, n}$-modules as in the previous section.

We begin by recalling a useful result of Wenzl's.

Lemma 8.1. (Wenzl [Wen88, Propositions 2.1(a) and 2.2(a)])

a) Any monomial $B_{\gamma} \in \mathscr{B}_{n}\left(\omega_{0}\right)$ is either in $\mathscr{B}_{n-1}(\omega)$ or it can be written in the form $a_{1} \alpha a_{2}$, where $a_{i} \in \mathscr{B}_{n-1}\left(\omega_{0}\right)$ and $\alpha \in\left\{E_{n-1}, S_{n-1}\right\}$.

b) $E_{n-1} \mathscr{B}_{n}\left(\omega_{0}\right) E_{n-1}=\mathscr{B}_{n-2}\left(\omega_{0}\right) E_{n-1}$.

LEMmA 8.2. Suppose that $n \geq 2$. Then $S_{n-1} \mathscr{B}_{n-1}\left(\omega_{0}\right) E_{n-1}=$ $\mathscr{B}_{n-1}\left(\omega_{0}\right) E_{n-1}$.

Proof. If $a \in \mathscr{B}_{n-2}\left(\omega_{0}\right)$ then $S_{n-1} a E_{n-1}=a S_{n-1} E_{n-1}=a E_{n-1}$. Suppose $a \notin \mathscr{B}_{n-2}\left(\omega_{0}\right)$. By Lemma 8.1, we can write $a=a_{1} \alpha a_{2}$ with $a_{i} \in \mathscr{B}_{n-2}\left(\omega_{0}\right)$ and $\alpha \in\left\{E_{n-2}, S_{n-2}\right\}$. If $\alpha=E_{n-2}$, then $S_{n-1} a E_{n-1}=$ $a_{1} S_{n-1} E_{n-2} E_{n-1} a_{2}=a_{1} S_{n-2} a_{2} E_{n-1}$. If $\alpha=S_{n-2}$ then $S_{n-1} a E_{n-1}=$ $a_{1} S_{n-1} S_{n-2} E_{n-1} a_{2}=a_{1} E_{n-2} a_{2} E_{n-1}$. In all cases we have $S_{n-1} a E_{n-1} \in$ $\mathscr{B}_{n-1}\left(\omega_{0}\right) E_{n-1}$. 
Lemma 8.3. Suppose that $n \geq 2$. Then for each $a \in \mathscr{W}_{r, n}$ there exists $h \in \mathscr{W}_{r, n-2}$ such that $\operatorname{deg} h \leq \operatorname{deg} a$ and $E_{n-1} a E_{n-1}=h E_{n-1}$. In particular, $E_{n-1} \mathscr{W}_{r, n} E_{n-1}=\mathscr{W}_{r, n-2} E_{n-1}$.

Proof. We argue by induction on $\operatorname{deg} a$. It is enough to consider the case where $a=X^{\alpha} B_{\gamma} X^{\beta}$ is an $r$-regular monomial in $\mathscr{W}_{r, n}$. Write $X^{\alpha}=$ $\dot{X}^{\alpha} X_{n-1}^{\alpha_{n-1}} X_{n}^{\alpha_{n}}$ and $X^{\beta}=\dot{X}^{\beta} X_{n-1}^{\beta_{n-1}} X_{n}^{\beta_{n}}$ and define $k=\alpha_{n-1}+\alpha_{n}+\beta_{n-1}+$ $\beta_{n}$. If $k=0$ then the result follows from Lemma 8.1(b), so we may assume that $k>0$. We split the proof into two cases.

Case 1. $B_{\gamma} \in \mathscr{B}_{n-1}\left(\omega_{0}\right):$ First suppose that $B_{\gamma} \in \mathscr{B}_{n-2}\left(\omega_{0}\right)$. Then we have

$$
\begin{aligned}
E_{n-1} X^{\alpha} B_{\gamma} X^{\beta} E_{n-1} & =\dot{X}^{\alpha} B_{\gamma} E_{n-1} X_{n-1}^{\alpha_{n-1}+\beta_{n-1}} X_{n}^{\alpha_{n}+\beta_{n}} E_{n-1} \dot{X}^{\beta} \\
& =(-1)^{\alpha_{n}+\beta_{n}} \dot{X}^{\alpha} B_{\gamma} E_{n-1} X_{n-1}^{k} E_{n-1} \dot{X}^{\beta}
\end{aligned}
$$

However, $E_{n-1} X_{n-1}^{k} E_{n-1}=\omega_{n-1}^{(k)} E_{n-1}$ by Lemma 4.15 , where $\omega_{n-1}^{(k)}$ is a central element in $\mathscr{W}_{r, n-2}$. If $k<r$ then $\operatorname{deg} \omega_{n-1}^{(k)}<k$ by Lemma 7.13, so the result follows by induction. Suppose then that $k \geq r$ then $X_{n-1}^{k}$ can be written as a linear combination of $r$-regular monomials of degree strictly less than $k$, so the result again follows by induction if $B_{\gamma} \in \mathscr{B}_{n-2}\left(\omega_{0}\right)$.

Next, suppose that $B_{\gamma} \notin \mathscr{B}_{n-2}\left(\omega_{0}\right)$. Then $B_{\gamma}=B_{\gamma^{\prime}} z B_{\gamma^{\prime \prime}}$, where $B_{\gamma^{\prime}}, B_{\gamma^{\prime \prime}} \in \mathscr{B}_{n-2}\left(\omega_{0}\right)$ and $z \in\left\{E_{n-2}, S_{n-2}\right\}$. So $E_{n-1} X^{\alpha} B_{\gamma} X^{\beta} E_{n-1}=$ $B_{\gamma^{\prime}} \dot{X}^{\alpha} E_{n-1} X_{n-1}^{\alpha_{n-1}} X_{n}^{\alpha_{n}} z X_{n-1}^{\beta_{n-1}} X_{n}^{\beta_{n}} E_{n-1} \dot{X}^{\beta} B_{\gamma^{\prime \prime}}$.

If $z=E_{n-2}$ then

$$
\begin{aligned}
& E_{n-1} X_{n-1}^{\alpha_{n-1}} X_{n}^{\alpha_{n}} E_{n-2} X_{n-1}^{\beta_{n-1}} X_{n}^{\beta_{n}} E_{n-1} \\
& = \pm E_{n-1} X_{n-1}^{\alpha_{n-1}+\alpha_{n}} E_{n-2} X_{n-1}^{\beta_{n-1}+\beta_{n}} E_{n-1} \\
& = \pm E_{n-1} X_{n-2}^{\alpha_{n-1}+\alpha_{n}} E_{n-2} X_{n-2}^{\beta_{n-1}+\beta_{n}} E_{n-1} \\
& = \pm X_{n-2}^{\alpha_{n-1}+\alpha_{n}} E_{n-1} E_{n-2} E_{n-1} X_{n-2}^{\beta_{n-1}+\beta_{n}} \\
& = \pm X_{n-2}^{\alpha_{n-1}+\alpha_{n}} E_{n-1} X_{n-2}^{\beta_{n-1}+\beta_{n}} \\
& = \pm X_{n-2}^{k} E_{n-1} .
\end{aligned}
$$

This completes the proof when $z=E_{n-2}$. 
Now suppose that $z=S_{n-2}$. Using the relations (2.1),

$$
\begin{aligned}
& E_{n-1} X_{n-1}^{\alpha_{n-1}} X_{n}^{\alpha_{n}} S_{n-2} X_{n-1}^{\beta_{n-1}} X_{n}^{\beta_{n}} E_{n-1} \\
& \quad= \pm E_{n-1} X_{n}^{\alpha_{n-1}+\alpha_{n}} S_{n-2} X_{n}^{\beta_{n-1}+\beta_{n}} E_{n-1} \\
& \quad= \pm E_{n-1} S_{n-2} X_{n}^{k} E_{n-1} \\
& \quad= \pm E_{n-1} E_{n-2} S_{n-1} X_{n}^{k} E_{n-1} .
\end{aligned}
$$

If $k \geq r$ then we can write $X_{n}^{k}$ as a linear combination of $r$-regular monomials each with degree strictly less than $k$. So by induction we may assume that $k<r$. Then, by Lemma 2.3, $S_{n-1} X_{n}^{k}=X_{n-1}^{k} S_{n-1}+X$, where $X \in \mathscr{W}_{r, n}$ is a linear combination of terms each of which has total degree in $X_{n}$ and $X_{n-1}$ strictly less than $k$. Hence, by induction, $E_{n-1} E_{n-2} X E_{n-1}=h^{\prime} E_{n-1}$, where $h^{\prime} \in \mathscr{W}_{r, n-2}$ and $\operatorname{deg} h^{\prime}<k$. Further,

$$
\begin{aligned}
E_{n-1} E_{n-2} X_{n-1}^{k} S_{n-1} E_{n-1} & =E_{n-1} E_{n-2} X_{n-1}^{k} E_{n-1} \\
& =(-1)^{k} E_{n-1} E_{n-2} X_{n-2}^{k} E_{n-1} \\
& =(-1)^{k} E_{n-1} E_{n-2} E_{n-1} X_{n-2}^{k} \\
& =(-1)^{k} X_{n-2}^{k} E_{n-1} .
\end{aligned}
$$

Consequently, $E_{n-1} X^{\alpha} B_{\gamma} X^{\beta} E_{n-1}=h E_{n-1}$, where $h \in \mathscr{W}_{r, n-2}$ and $\operatorname{deg} h \leq$ $\operatorname{deg} a$.

Case 2. $B_{\gamma} \notin \mathscr{B}_{n-1}\left(\omega_{0}\right)$ : Once again by Lemma 8.1 we can write $B_{\gamma}=$ $B_{\gamma^{\prime}} z B_{\gamma^{\prime \prime}}$, where $B_{\gamma^{\prime}}, B_{\gamma^{\prime \prime}} \in \mathscr{B}_{n-1}\left(\omega_{0}\right)$ and $z \in\left\{S_{n-1}, E_{n-1}\right\}$.

If $z=E_{n-1}$ then the result follows using Case 1 twice, so suppose that $z=S_{n-1}$. Then

$$
\begin{aligned}
& E_{n-1} X^{\alpha} B_{\gamma} X^{\beta} E_{n-1} \\
& \quad=\dot{X}^{\alpha} E_{n-1} X_{n-1}^{\alpha_{n-1}} X_{n}^{\alpha_{n}} B_{\gamma^{\prime}} S_{n-1} B_{\gamma^{\prime \prime}} X_{n-1}^{\beta_{n-1}} X_{n}^{\beta_{n}} E_{n-1} \dot{X}^{\beta} .
\end{aligned}
$$

If $\beta_{n-1}+\beta_{n}=0$ then $S_{n-1} B_{\gamma^{\prime \prime}} E_{n-1}=h E_{n-1}$, for some $h \in \mathscr{B}_{n-1}\left(\omega_{0}\right)$ by Lemma 8.2, so the result follows from Case 1. Hence, we may assume that $\beta_{n-1}+\beta_{n}>0$. Similarly, we may assume that $\alpha_{n-1}+\alpha_{n}>0$.

Next, suppose that $B_{\gamma^{\prime \prime}} \in \mathscr{B}_{n-2}\left(\omega_{0}\right)$. Then

$$
\begin{aligned}
& E_{n-1} X^{\alpha} B_{\gamma} X^{\beta} E_{n-1} \\
& \quad= \pm \dot{X}^{\alpha} E_{n-1} X_{n-1}^{\alpha_{n-1}+\alpha_{n}} B_{\gamma^{\prime}} B_{\gamma^{\prime \prime}} S_{n-1} X_{n}^{\beta_{n-1}+\beta_{n}} E_{n-1} \dot{X}^{\beta}
\end{aligned}
$$


Once again, by induction we may assume that $\beta_{n-1}+\beta_{n}<r$. Then, by Lemma $2.3 S_{n-1} X_{n}^{\beta_{n-1}+\beta_{n}}=X_{n-1}^{\beta_{n-1}+\beta_{n}} S_{n-1}+X$, where $\operatorname{deg} X<\beta_{n-1}+$ $\beta_{n}$. As $S_{n-1} E_{n-1}=E_{n-1}$ it is enough to consider $E_{n-1} X_{n-1}^{\alpha_{n-1}+\alpha_{n}} B_{\gamma^{\prime}} B_{\gamma^{\prime \prime}}$ $X_{n-1}^{\beta_{n-1}+\beta_{n}} E_{n-1}$. As $B_{\gamma^{\prime}} B_{\gamma^{\prime \prime}} \in \mathscr{B}_{n-1}\left(\omega_{0}\right)$ this can be written in the required form by Case 1 .

Finally, suppose that $B_{\gamma^{\prime \prime}} \notin \mathscr{B}_{n-2}\left(\omega_{0}\right)$. Then either $B_{\gamma^{\prime \prime}}=B_{\gamma_{1}^{\prime \prime}} E_{n-2}$ $B_{\gamma_{2}^{\prime \prime}}$, or $B_{\gamma^{\prime \prime}}=B_{\gamma_{1}^{\prime \prime}} S_{n-2} B_{\gamma_{2}^{\prime \prime}}$, where $B_{\gamma_{1}^{\prime \prime}}, B_{\gamma_{2}^{\prime \prime}} \in \mathscr{B}_{n-2}\left(\omega_{0}\right)$. If $B_{\gamma^{\prime \prime}}=$ $B_{\gamma_{1}^{\prime \prime}} E_{n-2} B_{\gamma_{2}^{\prime \prime}}$ then

$$
\begin{aligned}
& E_{n-1} X_{n-1}^{\alpha_{n-1}} X_{n}^{\alpha_{n}} B_{\gamma^{\prime}} S_{n-1} B_{\gamma_{1}^{\prime \prime}} E_{n-2} B_{\gamma_{2}^{\prime \prime}} X_{n-1}^{\beta_{n-1}} X_{n}^{\beta_{n}} E_{n-1} \\
& = \pm E_{n-1} X_{n}^{\alpha_{n-1}+\alpha_{n}} B_{\gamma^{\prime}} B_{\gamma_{1}^{\prime \prime}} S_{n-1} E_{n-2} B_{\gamma_{2}^{\prime \prime}} X_{n-1}^{\beta_{n-1}+\beta_{n}} E_{n-1} \\
& = \pm E_{n-1} X_{n}^{\alpha_{n-1}+\alpha_{n}} B_{\gamma^{\prime}} B_{\gamma_{1}^{\prime \prime}} S_{n-1} E_{n-2} X_{n-1}^{\beta_{n-1}+\beta_{n}} E_{n-1} B_{\gamma_{2}^{\prime \prime}} \\
& = \pm E_{n-1} X_{n}^{\alpha_{n-1}+\alpha_{n}} B_{\gamma^{\prime}} B_{\gamma_{1}^{\prime \prime}} S_{n-1} E_{n-2} X_{n-2}^{\beta_{n-1}+\beta_{n}} E_{n-1} B_{\gamma_{2}^{\prime \prime}} \\
& = \pm E_{n-1} X_{n}^{\alpha_{n-1}+\alpha_{n}} B_{\gamma^{\prime}} B_{\gamma_{1}^{\prime \prime}} S_{n-1} E_{n-2} E_{n-1} X_{n-2}^{\beta_{n-1}+\beta_{n}} B_{\gamma_{2}^{\prime \prime}} \\
& = \pm E_{n-1} X_{n}^{\alpha_{n-1}+\alpha_{n}} B_{\gamma^{\prime}} B_{\gamma_{1}^{\prime \prime}} S_{n-2} E_{n-1} X_{n-2}^{\beta_{n-1}+\beta_{n}} B_{\gamma_{2}^{\prime \prime}} .
\end{aligned}
$$

Now $\operatorname{deg}\left(X^{\alpha} B_{\gamma^{\prime}} B_{\gamma_{1}^{\prime \prime}} S_{n-2}\right)<\operatorname{deg} a$ since $\beta_{n-1}+\beta_{n}>0$. Hence, the result now follows by induction. If $B_{\gamma^{\prime \prime}}=B_{\gamma_{1}^{\prime \prime}} S_{n-2} B_{\gamma_{2}^{\prime \prime}}$ then

$$
\begin{aligned}
& E_{n-1} X_{n-1}^{\alpha_{n-1}} X_{n}^{\alpha_{n}} B_{\gamma^{\prime}} S_{n-1} B_{\gamma_{1}^{\prime \prime}} S_{n-2} B_{\gamma_{2}^{\prime \prime}} X_{n-1}^{\beta_{n-1}} X_{n}^{\beta_{n}} E_{n-1} \\
& \quad= \pm E_{n-1} X_{n}^{\alpha_{n-1}+\alpha_{n}} B_{\gamma^{\prime}} B_{\gamma_{1}^{\prime \prime}} S_{n-1} S_{n-2} X_{n}^{\beta_{n-1}+\beta_{n}} E_{n-1} B_{\gamma_{2}^{\prime \prime}} \\
& \quad= \pm E_{n-1} X_{n}^{\alpha_{n-1}+\alpha_{n}} B_{\gamma^{\prime}} B_{\gamma_{1}^{\prime \prime}} S_{n-1} X_{n}^{\beta_{n-1}+\beta_{n}} S_{n-2} E_{n-1} B_{\gamma_{2}^{\prime \prime}}
\end{aligned}
$$

By Lemma 2.3 we can write $S_{n-1} X_{n}^{\beta_{n-1}+\beta_{n}}=X_{n-1}^{\beta_{n-1}+\beta_{n}} S_{n-1}+X$, where $\operatorname{deg} X<\beta_{n-1}+\beta_{n}$. Now,

$$
\begin{aligned}
& E_{n-1} X_{n}^{\alpha_{n-1}+\alpha_{n}} B_{\gamma^{\prime}} B_{\gamma_{1}^{\prime \prime}} X_{n-1}^{\beta_{n-1}+\beta_{n}} S_{n-1} S_{n-2} E_{n-1} B_{\gamma_{2}^{\prime \prime}} \\
& =E_{n-1} B_{\gamma^{\prime}} B_{\gamma_{1}^{\prime \prime}} X_{n-1}^{\beta_{n-1}+\beta_{n}} X_{n}^{\alpha_{n-1}+\alpha_{n}} E_{n-2} E_{n-1} B_{\gamma_{2}^{\prime \prime}} \\
& =E_{n-1} B_{\gamma^{\prime}} B_{\gamma_{1}^{\prime \prime}} X_{n-1}^{\beta_{n-1}+\beta_{n}} E_{n-2} X_{n}^{\alpha_{n-1}+\alpha_{n}} E_{n-1} B_{\gamma_{2}^{\prime \prime}} \\
& =E_{n-1} B_{\gamma^{\prime}} B_{\gamma_{1}^{\prime \prime}} X_{n-1}^{\beta_{n-1}+\beta_{n}} E_{n-2} X_{n-2}^{\alpha_{n-1}+\alpha_{n}} E_{n-1} B_{\gamma_{2}^{\prime \prime}} \\
& =E_{n-1} B_{\gamma^{\prime}} B_{\gamma_{1}^{\prime \prime}} X_{n-1}^{\beta_{n-1}+\beta_{n}} E_{n-2} E_{n-1} B_{\gamma_{2}^{\prime \prime}} X_{n-2}^{\alpha_{n-1}+\alpha_{n}} .
\end{aligned}
$$

As $\alpha_{n-1}+\alpha_{n}>0$ we can write $E_{n-1} B_{\gamma^{\prime}} B_{\gamma_{1}^{\prime \prime}} X_{n-1}^{\beta_{n-1}+\beta_{n}} E_{n-2} E_{n-1}$ in the required form and so completes the proof of the case - and hence the Lemma. 
By iterating the Lemma we obtain the result that we really want.

Corollary 8.4. Suppose $f>0, w \in \mathfrak{S}_{n}$ and that $\kappa, \rho \in \mathbb{N}_{r}^{(f)}$. Then

$$
E^{f} X^{\rho} S_{w} X^{\kappa} E^{f}=h E^{f}
$$

for some $h \in \mathscr{W}_{r, n-2 f}$.

As we now briefly recall, by the general theory of cellular algebras [GL96], [Mat99], every irreducible $\mathscr{W}_{r, n}$-module arises in a unique way as the simple head of some cell module. For each $(f, \lambda) \in \Lambda_{r}^{+}$fix $(\mathfrak{s}, \rho, e) \in \delta(f, \lambda)$ and let $C_{(\mathfrak{t}, \kappa, d)}^{(f, \lambda)}=C_{(\mathfrak{s}, \rho, e)(\mathfrak{t}, \kappa, d)}^{(f, \lambda)}+\mathscr{W}_{r, n}^{\triangleright \lambda}$. By Theorem 7.17 the cell modules of $\mathscr{W}_{r, n}$ are the modules $\Delta(f, \lambda)$ which are the free $R$-modules with basis $\left\{C_{(\mathfrak{t}, \kappa, d)}^{(f, \lambda)} \mid(\mathfrak{t}, \kappa, d) \in \delta(f, \lambda)\right\}$. The cell module $\Delta(f, \lambda)$ comes equipped with a natural bilinear form $\phi_{f, \lambda}$ which is determined by the equation

$$
\begin{aligned}
& C_{(\mathfrak{s}, \rho, e)(\mathfrak{t}, \kappa, d)}^{(f, \lambda)} C_{\left(\mathfrak{t}^{\prime}, \kappa^{\prime}, d^{\prime}\right)(\mathfrak{s}, \rho, e)}^{(f, \lambda)} \\
& \quad \equiv \phi_{f, \lambda}\left(C_{(\mathfrak{t}, \kappa, d)}^{(f, \lambda)}, C_{\left(\mathfrak{t}^{\prime}, \kappa^{\prime}, d^{\prime}\right)}^{(f, \lambda)}\right) \cdot C_{(\mathfrak{s}, \rho, e)(\mathfrak{s}, \rho, e)}^{(f, \lambda)} \quad\left(\bmod \mathscr{W}_{r, n}^{\triangleright \lambda}\right) .
\end{aligned}
$$

The form $\phi_{f, \lambda}$ is $\mathscr{W}_{r, n}$-invariant in the sense that $\phi_{f, \lambda}(x a, y)=\phi_{f, \lambda}\left(x, y a^{*}\right)$, for $x, y \in \Delta(f, \lambda)$ and $a \in \mathscr{W}_{r, n}$. Consequently,

$$
\operatorname{Rad} \Delta(f, \lambda)=\left\{x \in \Delta(f, \lambda) \mid \phi_{f, \lambda}(x, y)=0 \text { for all } y \in \Delta(f, \lambda)\right\}
$$

is a $\mathscr{W}_{r, n}$-submodule of $\Delta(f, \lambda)$ and $D(f, \lambda)=\Delta(f, \lambda) / \operatorname{Rad} \Delta(f, \lambda)$ is either zero or absolutely irreducible.

In exactly the same way, for each multipartition $\lambda \in \Lambda_{r}^{+}(n-2 f)$ the corresponding cell module $S(\lambda)$ for $\mathscr{H}_{r, n-2 f}$, the Specht module of Section 6 , carries a bilinear form $\phi_{\lambda}$. The quotient module $D(\lambda)=S(\lambda) / \operatorname{Rad} S(\lambda)$ is either zero or an absolutely irreducible $\mathscr{H}_{r, n-2 f}$-module.

We can now prove Theorem $\mathrm{C}$.

TheOREm 8.5. Suppose that $R$ is a field in which 2 is invertible, that $\Omega$ is $\mathbf{u}$-admissible and that $\omega_{0} \neq 0$. Let $(f, \lambda) \in \Lambda_{r}^{+}$. Then $D^{(f, \lambda)} \neq 0$ if and only if $D^{\lambda} \neq 0$.

Proof. It is enough to prove that $\phi_{f, \lambda} \neq 0$ if and only if $\phi_{\lambda} \neq 0$. 
First, suppose that $\phi_{\lambda} \neq 0$. Recall that the Specht module $S(\lambda)$ has basis $\left\{m_{\mathfrak{t}} \mid \mathfrak{t} \in \mathscr{T}^{s t d}(\lambda)\right\}$. Then $\phi_{\lambda}\left(m_{\mathfrak{t}}, m_{\mathfrak{v}}\right) \neq 0$, for some $\mathfrak{t}, \mathfrak{v} \in \mathscr{T}^{\text {std }}(\lambda)$; that is, $m_{\mathfrak{s t}} m_{\mathfrak{v} \mathfrak{f}} \notin \mathscr{H}_{r, n-2 f}^{\triangleright \lambda}$. Let $\underline{0}$ to the zero vector in $\mathbb{N}_{r}^{(f)}$. Then

$$
\begin{aligned}
C_{(\mathfrak{s}, \rho, e)(\mathfrak{t}, \underline{0}, 1)}^{(f, \lambda)} C_{(\mathfrak{v}, \underline{0}, 1)(\mathfrak{s}, \rho, e)}^{(f, \lambda)} & =S_{e}^{*} X^{\rho} E^{f} M_{\mathfrak{s t}} E^{f} M_{\mathfrak{v} \mathfrak{s}} X^{\rho} S_{e} \\
& =S_{e}^{*} X^{\rho}\left(E^{f}\right)^{2} M_{\mathfrak{s t}} M_{\mathfrak{v} \mathfrak{s}} X^{\rho} S_{e} \\
& \equiv \omega_{0}^{f} \phi_{\lambda}\left(m_{\mathfrak{t}}, m_{\mathfrak{v}}\right) S_{e}^{*} X^{\rho} E^{f} M_{\mathfrak{s} \mathfrak{s}} X^{\rho} S_{e} \\
& \equiv \omega_{0}^{f} \phi_{\lambda}\left(m_{\mathfrak{t}}, m_{\mathfrak{v}}\right) C_{(\mathfrak{s}, \rho, e)(\mathfrak{s}, \rho, e)}^{(f, \lambda)} \quad\left(\bmod \mathscr{W}_{r, n}^{\triangleright \lambda}\right) .
\end{aligned}
$$

Hence, $\phi_{f, \lambda}\left(C_{(\mathfrak{t}, \underline{0}, 1)}^{(f, \lambda)}, C_{(\mathfrak{v}, \underline{0}, 1)}^{(f, \lambda)}\right)=\omega_{0}^{f} \phi_{\lambda}\left(m_{\mathfrak{t}}, m_{\mathfrak{v}}\right) \neq 0$, so that $\phi_{f, \lambda} \neq 0$.

Now suppose that $\phi_{f, \lambda} \neq 0$. Then there exist $(\mathfrak{u}, \alpha, u),\left(\mathfrak{v}, \beta^{\prime}, v\right) \in$ $\delta(f, \lambda)$ such that $\phi_{f, \lambda}\left(C_{(\mathfrak{u}, \alpha, u)}^{(f, \lambda)}, C_{(\mathfrak{v}, \beta, v)}^{(f, \lambda)}\right) \neq 0$. That is,

$$
\begin{aligned}
0 & \neq C_{(\mathfrak{s}, \rho, e)(\mathfrak{u}, \alpha, u)}^{(f, n)} \cdot C_{(\mathfrak{v}, \beta, v)(\mathfrak{s}, \rho, e)}^{(f, \lambda)} \\
& =S_{e}^{*} X^{\rho} E^{f} M_{\mathfrak{s u}} X^{\alpha} S_{u} \cdot S_{v}^{*} X^{\beta} E^{f} M_{\mathfrak{v g}} X^{\rho} S_{e} \\
& =S_{e}^{*} X^{\rho} M_{\mathfrak{s u}} E^{f} X^{\alpha} S_{u} S_{v}^{*} X^{\beta} E^{f} M_{\mathfrak{v} \mathfrak{s}} X^{\rho} S_{e} \\
& =S_{e}^{*} X^{\rho} M_{\mathfrak{s u}} h M_{\mathfrak{v} \mathfrak{s}} E^{f} X^{\rho} S_{e},
\end{aligned}
$$

for some $h \in \mathscr{W}_{r, n-2 f}$ by Corollary 8.4. Now, $M_{\mathfrak{s u}} \mathcal{E}_{f} M_{\mathfrak{v g}} E^{f} \subseteq \mathcal{E}_{f} E^{f} \subseteq$ $\mathscr{W}_{r, n}^{f+1}$, by Lemma 7.4. Therefore, Proposition 7.2 implies that there is an $h^{\prime} \in \mathscr{H}_{r, n-2 f}$ such that $m_{\mathfrak{s u}} h^{\prime} m_{\mathfrak{v s}} \neq 0\left(\bmod \mathscr{H}_{r, n-2 f}^{\triangleright \lambda}\right)$. Consequently, $\phi_{\lambda} \neq 0$. This completes the proof of the Theorem.

We remark that the irreducible representations of the Ariki-Koike algebras are indexed by the $\mathbf{u}$-Kleshchev multipartitions; see [Ari01], [AM00]. In the special case when $u_{i}=d_{i} \cdot 1_{R}$, for $1 \leq i \leq r$ and where $0 \leq d_{i}<\operatorname{char} R$, Kleshchev [Kle05] has shown that the simple $\mathscr{H}_{r, n}(\mathbf{u})$-modules are labelled by a set of multipartitions which gives the same Kashiwara crystal as the set of $\mathbf{u}$-Kleshchev multipartitions of $n$. Hence, in this case, the simple $\mathscr{W}_{r, n}(\mathbf{u})$-modules are labelled by the set $\{(f, \lambda)\}$, where $0 \leq f \leq\left\lfloor\frac{n}{2}\right\rfloor$ and $\lambda$ is a $\mathbf{u}$-Kleshchev multipartition of $n-2 f$. By modifying the proof of [DM02, Theorem 1.1], or [AM00, Theorem 1.3], one can show that under the assumptions of Theorem 8.5 the simple $\mathscr{W}_{r, n}(\mathbf{u})$-modules are always labelled by the $\mathbf{u}$-Kleshchev multipartitions. (Note, however, that we are not claiming that $D^{(f, \lambda)} \neq 0$ for the multipartitions $\lambda$ which Kleshchev [Kle05] uses to label the irreducible $\mathscr{H}_{r, n}(\mathbf{u})$-modules.) 
We close by classifying the quasi-hereditary cyclotomic Nazarov-Wenzl algebras with $\omega_{0} \neq 0$. See [CPS88] for the definition of a quasi-hereditary algebra.

COROllary 8.6. Suppose that $R$ is a field in which 2 is invertible, that $\Omega$ is $\mathbf{u}$-admissible and that $\omega_{0} \neq 0$. Then $\mathscr{W}_{r, n}(\mathbf{u})$ is a quasi-hereditary algebra if and only if char $R>n$ and $\mathbf{u}$ is generic for $\mathscr{H}_{r, n}(\mathbf{u})$ (Definition 6.4).

Proof. By [GL96, (3.10)], a cellular algebra is quasi-hereditary if and only if the bilinear form on each cell module does not vanish. Therefore, $\mathscr{W}_{r, n}$ is a quasi-hereditary algebra if and only if $D^{(f, \lambda)} \neq 0$ for all $(f, \lambda) \in \Lambda_{r}^{+}$and $\mathscr{H}_{r, n-2 f}(\mathbf{u})$ is quasi-hereditary if and only if $D^{\lambda} \neq 0$ for all $\lambda \in \Lambda_{r}^{+}(n-2 f)$. Hence, by Theorem 8.5, $\mathscr{W}_{r, n}(\mathbf{u})$ is quasi-hereditary if and only if the algebras $\mathscr{H}_{r, n-2 f}(\mathbf{u})$ are all quasi-hereditary, for $0 \leq f \leq\left\lfloor\frac{n}{2}\right\rfloor$. However, the degenerate Hecke algebras are Frobenius algebras by [Kle05, Cor. 5.7.4], so they are quasi-hereditary precisely when they are semisimple - since Frobenius algebras have infinite global dimension when they are not semisimple, whereas quasi-hereditary algebras have finite global dimension (see [Don99, Prop. A2.3]). Hence the result follows from Theorem 6.11.

\section{REFERENCES}

[AK94] S. Ariki and K. Koike, A Hecke algebra of $(\mathbf{Z} / r \mathbf{Z}) \imath \mathfrak{S}_{n}$ and construction of its irreducible representations, Adv. Math., 106 (1994), 216-243.

[AM00] S. Ariki and A. Mathas, The number of simple modules of the Hecke algebras of type $G(r, 1, n)$, Math. Z., 233 (2000), 601-623.

[Ari96] S. Ariki, On the decomposition numbers of the Hecke algebra of $G(m, 1, n)$, J. Math. Kyoto Univ., 36 (1996), 789-808.

[Ari01] - On the classification of simple modules for cyclotomic Hecke algebras of type $G(m, 1, n)$ and Kleshchev multipartitions, Osaka J. Math., 38 (2001), 827-837.

[Bra37] R. Brauer, On algebras which are connected with the semisimple continuous groups, Ann. of Math., 38 (1937), 857-872.

[Bro56] W. P. Brown, The semisimplicity of $\omega_{f}^{n}$, Ann. of Math. (2), 63 (1956), 324-335.

[BW89] J. S. Birman and H. Wenzl, Braids, link polynomials and a new algebra, Trans. Amer. Math. Soc., 313 (1989), 249-273.

[CGW05] A. M. Cohen, D. A. H. Gijsbers and D. B. Wales, BMW algebras of simply laced type, J. Algebra, 286 (2005), 107-153.

[CPS88] E. Cline, B. Parshall and L. Scott, Finite-dimensional algebras and highest weight categories, J. Reine Angew. Math., 391 (1988), 85-99.

[DJM99] R. Dipper, G. James and A. Mathas, Cyclotomic q-Schur algebras, Math. Z., 229 (1999), 385-416. 
[DM02] R. Dipper and A. Mathas, Morita equivalences of Ariki-Koike algebras, Math. Z., 240 (2002), 579-610.

[Don99] S. Donkin, The $q$-Schur algebra, Lond. Math. Soc. Lecture Notes, 253, CUP, Cambridge, 1999.

[DWH99] W. F. Doran, IV, D. B. Wales and P. J. Hanlon, On the semisimplicity of the Brauer centralizer algebras, J. Algebra, 211 (1999), 647-685.

[Eny04] J. Enyang, Cellular bases for the Brauer and Birman-Murakami-Wenzl algebras, J. Algebra, 281 (2004), 413-449.

[FG95] S. Fishel and I. Grojnowski, Canonical bases for the Brauer centralizer algebra, Math. Res. Lett., 2 (1995), 15-26.

[GH] F. M. Goodman and H. M. Hauschild, Affine Birman-Wenzl-Murakami Algebras and Tangles in the Solid Torus, arXiv:math.QA/0411155.

[GL96] J. J. Graham and G. I. Lehrer, Cellular algebras, Invent. Math., 123 (1996), $1-34$.

[Gre80] J. A. Green, Polynomial representations of $g l_{n}$, SLN, 830, Springer-Verlag, New York, 1980.

[HO01] R. Häring-Oldenburg, Cyclotomic Birman-Murakami-Wenzl algebras, J. Pure Appl. Algebra, 161 (2001), 113-144.

[HR95] T. Halverson and A. Ram, Characters of algebras containing a Jones basic construction: the Temperley-Lieb, Okasa, Brauer, and Birman-Wenzl algebras, Adv. Math., 116 (1995), 263-321.

[HW89a] P. Hanlon and D. Wales, Eigenvalues connected with Brauer's centralizer algebras, J. Algebra, 121 (1989), 446-476.

[HW89b] On the decomposition of Brauer's centralizer algebra, J. Algebra, 121 (1989), 409-445.

[JM00] G. D. James and A. Mathas, The Jantzen sum formula for cyclotomic q-Schur algebras, Trans. Amer. Math. Soc., 352 (2000), 5381-5404.

[Jon94] V. F. R. Jones, A quotient of the affine Hecke algebra in the Brauer algebra, 'Enseign. Math., 40 (1994), 313-344.

[Kle05] A. S. Kleshchev, Lectures on linear and projective representations of symmetric groups, CUP, 2005.

[LR97] R. Leduc and A. Ram, A ribbon Hopf algebra approach to the irreducible representations of centralizer algebras: the Brauer, Birman-Wenzl, and type $A$ Iwahori-Hecke algebras, Adv. Math., 125 (1997), 1-94.

[Mac95] I. G. Macdonald, Symmetric functions and Hall polynomials, second éd., Oxford Mathematical Monographs, Clarendon Press, Oxford, 1995.

[Mar96] P. Martin, The structure of the partition algebras, J. Algebra, 183 (1996), 319-358.

[Mat99] A. Mathas, Hecke algebras and Schur algebras of the symmetric group, Univ. Lecture Notes, 15, Amer. Math. Soc., 1999.

[Mat04] Matrix units and generic degrees for the Ariki-Koike algebras, J. Algebra, 281 (2004), 695-730.

[Mur83] G. E. Murphy, The idempotents of the symmetric group and Nakayama's conjecture, J. Algebra, 81 (1983), 258-265. 
[MW00] H. R. Morton and A. J. Wassermann, A basis for the Birman-Wenzl algebra, preprint (2000).

[Naz96] M. Nazarov, Young's orthogonal form for Brauer's centralizer algebra, J. Algebra, 182 (1996), 664-693.

[OR] R. Orellana and A. Ram, Affine braids, Markov traces and the category O, arXiv:math.RT/0401317.

[PK98] M. Parvathi and M. Kamaraj, Signed Brauer's algebras, Comm. Algebra, 26 (1998), 839-855.

[PK02] Matrix units for signed Brauer's algebras, Southeast Asian Bull. Math., 26 (2002), 279-297.

[PS02] M. Parvathi and D. Savithri, Representations of G-Brauer algebras, Southeast Asian Bull. Math., 26 (2002), 453-468.

[Ram95] A. Ram, Characters of Brauer's centralizer algebras, Pacific J. Math., 169 (1995), 173-200.

[Rui05] H. Rui, A criterion on semisimple Brauer algebra, J. Comb. Theory, Ser. A, 111 (2005), 78-88.

[RY04] H. Rui and W. Yu, On the semisimplicity of cyclotomic Brauer algebras, J. Algebra, 277 (2004), 187-221.

[Sun86] S. Sundaram, On the combinatorics of representations of $S p(2 n, C)$, Thèse, Massachusetts Institute of Technology (1986).

[Ter01] I. Terada, Brauer diagrams, updown tableaux and nilpotent matrices, J. Algebraic Combin., 14 (2001), 229-267.

[Wen88] H. Wenzl, On the structure of Brauer's centralizer algebras, Ann. of Math., 128 (1988), 173-193.

[Wey97] H. Weyl, The classical groups, Princeton Landmarks in Mathematics, Princeton University Press, Princeton, NJ, 1997, Their invariants and representations, Fifteenth printing, Princeton Paperbacks.

[Xi00] C. Xi, On the quasi-heredity of Birman-Wenzl algebras, Adv. Math., 154 (2000), 280-298.

\author{
Susumu Ariki \\ Research Institute for Mathematical Sciences \\ Kyoto University \\ Kyoto, 606-8502 \\ Japan \\ ariki@kurims.kyoto-u.ac.jp \\ Andrew Mathas \\ School of Mathematics and Statistics F07 \\ University of Sydney \\ NSW 2006 \\ Australia \\ a.mathas@maths.usyd.edu.au
}


Hebing Rui

Department of Mathematics

East China Normal University

200062 Shanghai

P. R. China

hbrui@math.ecnu.edu.cn 